\title{
Synthesis of heteroaryl imines: theoretical and experimental approach to the determination of
}

\section{configuration of $\mathrm{C}=\mathrm{N}$ double bond}

\author{
Mario Amati, Carlo Bonini, Maurizio D’Auria, * Maria Funicello, Francesco Lelj and Rocco \\ Racioppi.
}

\section{Table of contents}

Synthesi of imines and photolysis - general procedures

S2

${ }^{1} \mathrm{H}$ NMR, ${ }^{13} \mathrm{C}$ NMR of compound 3a-d, 5a-b, 6a-b, 7a-b, 8, 9

S3-S23

Cartesian coordinates, frequencies, total energy of compound 3a-d, 5a-b, 6a-b, 7a-b

S24-S66 
Column chromatography was carried out on silica gel $(0.063-0.200 \mathrm{~mm}$ particle size) by progressive elution with petroleum ether/ethyl acetate mixtures. ${ }^{1} \mathrm{H}$ and ${ }^{13} \mathrm{C}$ NMR spectra were normally carried out in $\mathrm{CDCl}_{3}$ solutions on a $300 \mathrm{MHz}$ and/or on $500 \mathrm{MHz}$ instruments. Mass spectra were obtained with a mass-selective detector on a gas chromatograph (OV-1 capillary column between $70-250{ }^{\circ} \mathrm{C}\left(20^{\circ} \mathrm{C} / \mathrm{min}\right)$. Toluene was dried on $\mathrm{Na}$ /benzophenone. All the heteroaromatic aldehydes used in this work were purchased.

$N$-(2-benzothienyl)diphenylmethyliminophosphorane and the opportune azidic precursor were synthesized as previously reported. ${ }^{4}$

Synthesis of imines: general procedure at $60^{\circ} \mathrm{C}$

Equimolar amounts of benzothienyliminophosphorane and of the opportune heteroaryl aldehyde were stirred in dry toluene and in nitrogen atmosphere at $60{ }^{\circ} \mathrm{C}$ for about 24 hours. After this time the reaction mixture was purified on silica gel column (eluent 8:2 hexanes/ethyl acetate). The purification furnished an iminic product identified by ${ }^{1} \mathrm{H}$ and ${ }^{13} \mathrm{C}$ NMR, UV spectra in chloroform solution and mass spectroscopy.

Synthesis of imines: general procedure at $100{ }^{\circ} \mathrm{C}$

Equimolar amounts of benzothienyliminophosphorane and of the opportune heteroaryl aldehyde were stirred in dry toluene and in nitrogen atmosphere at $100{ }^{\circ} \mathrm{C}$ for about 24 hours. After this time the reaction mixture was purified on silica gel column (eluent 8:2 hexanes/ethyl acetate). The purification furnished two iminic products identified by ${ }^{1} \mathrm{H}$ and ${ }^{13} \mathrm{C} N M R$, UV spectra in chloroform solution and mass spectroscopy.

Photolysis of iminic products: general procedure

A chloroform solution of the imine (10 $\mathrm{mg}$ in $20 \mathrm{ml}$ of chloroform) was flushed with nitrogen for 1 h. The mixture was then irradiated in Pyrex recipient at room temperature by a $125 \mathrm{~W}$ high pressure mercuric lamp (Helios-Italquatz, Milan) until disappeareance of substrate. The reaction was monitored by tlc (eluent: 8/2 hexanes/ethyl acetate). After solvent removal the crude mixture was purified by column chromatography on silica gel (eluent 8/2 hexanes/ethyl acetate) to obtain a tetracyclic compound characterized by GC/MS and NMR spectroscopy. 


\section{Compound $3 a-{ }^{1} \mathrm{H}$ NMR spectrum}

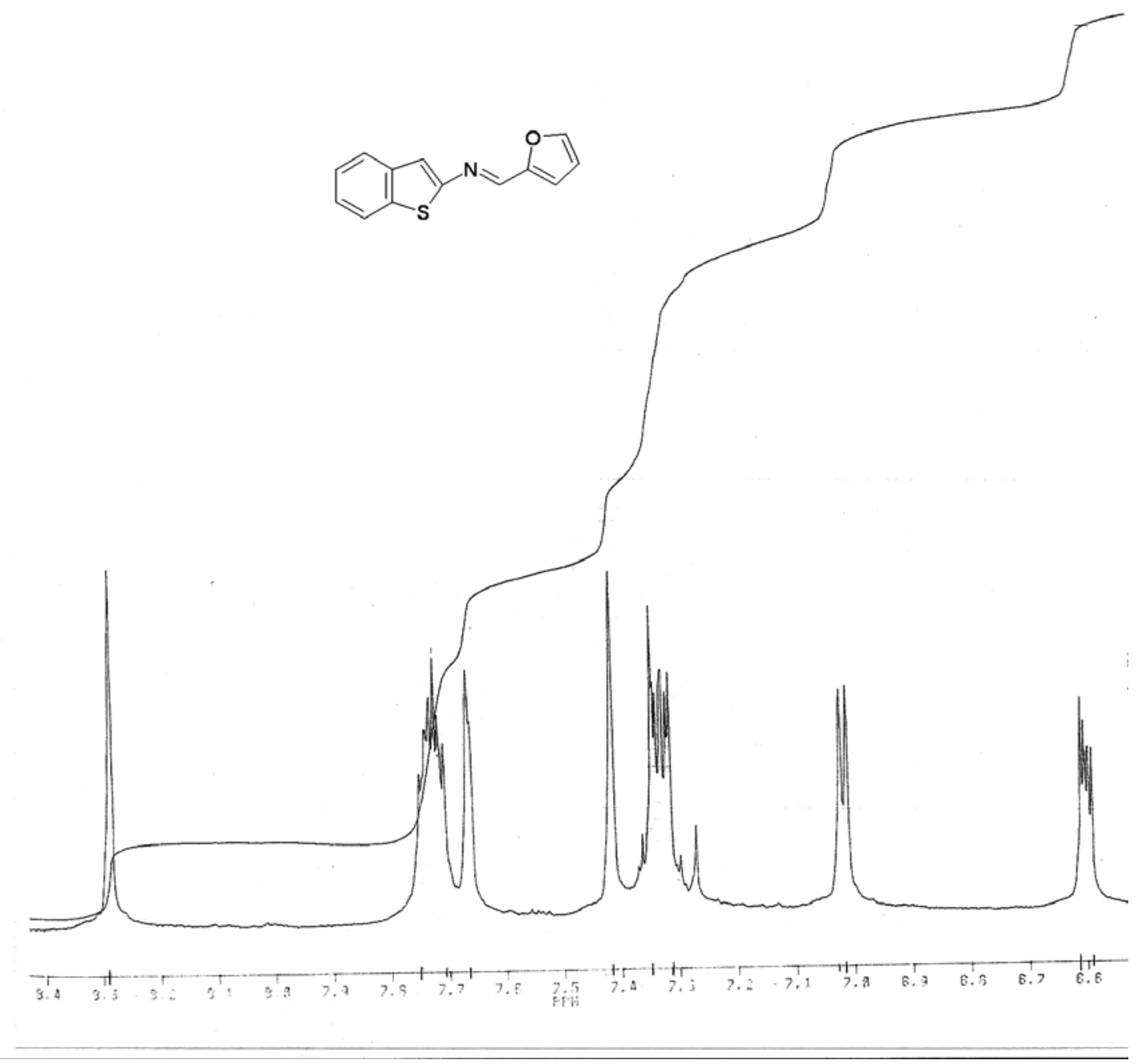




\section{Compound 3a $-{ }^{13} \mathrm{C}$ NMR spectrum}

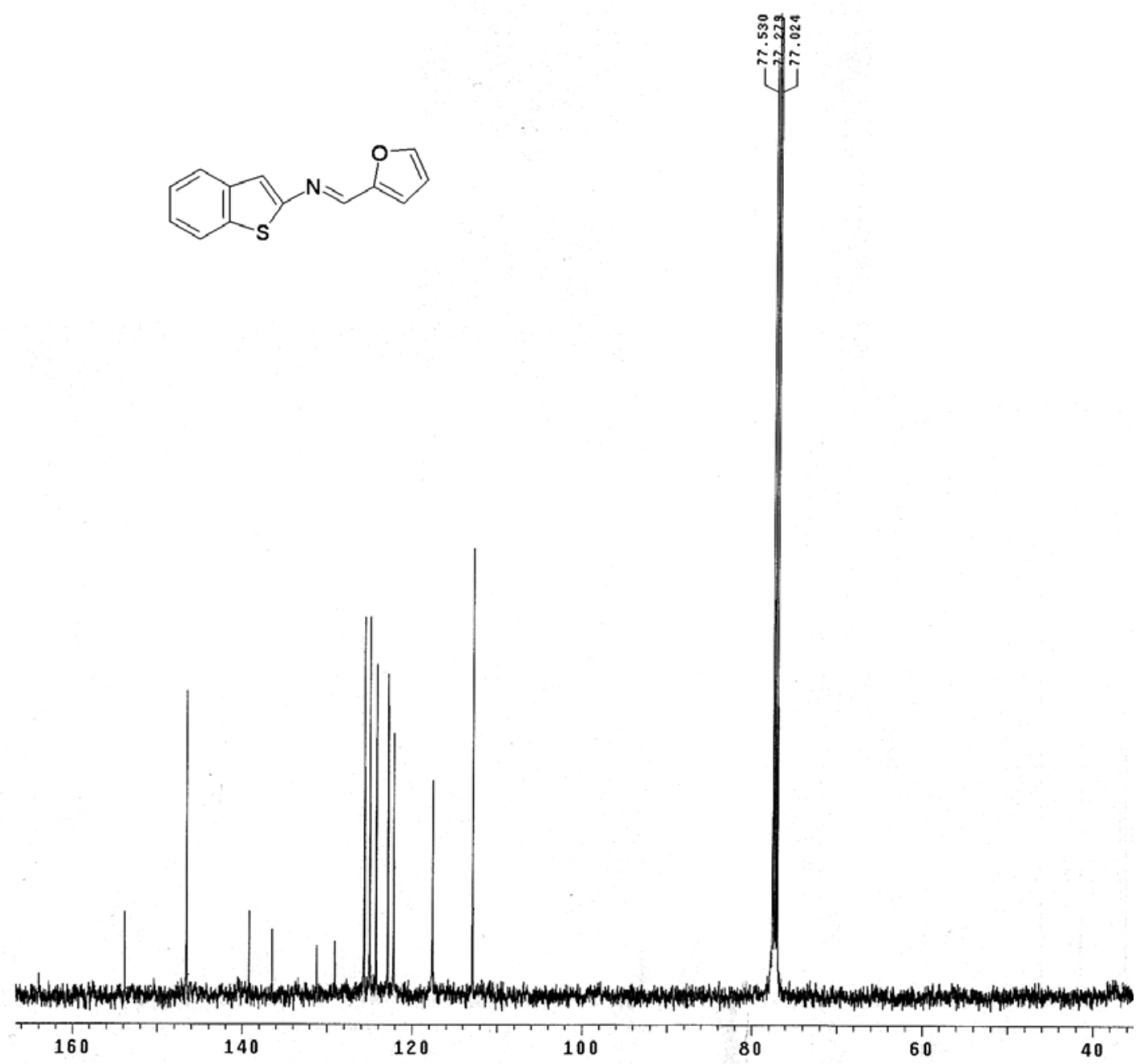




\section{Compound $3 b$ - ${ }^{1}$ H NMR spectrum}

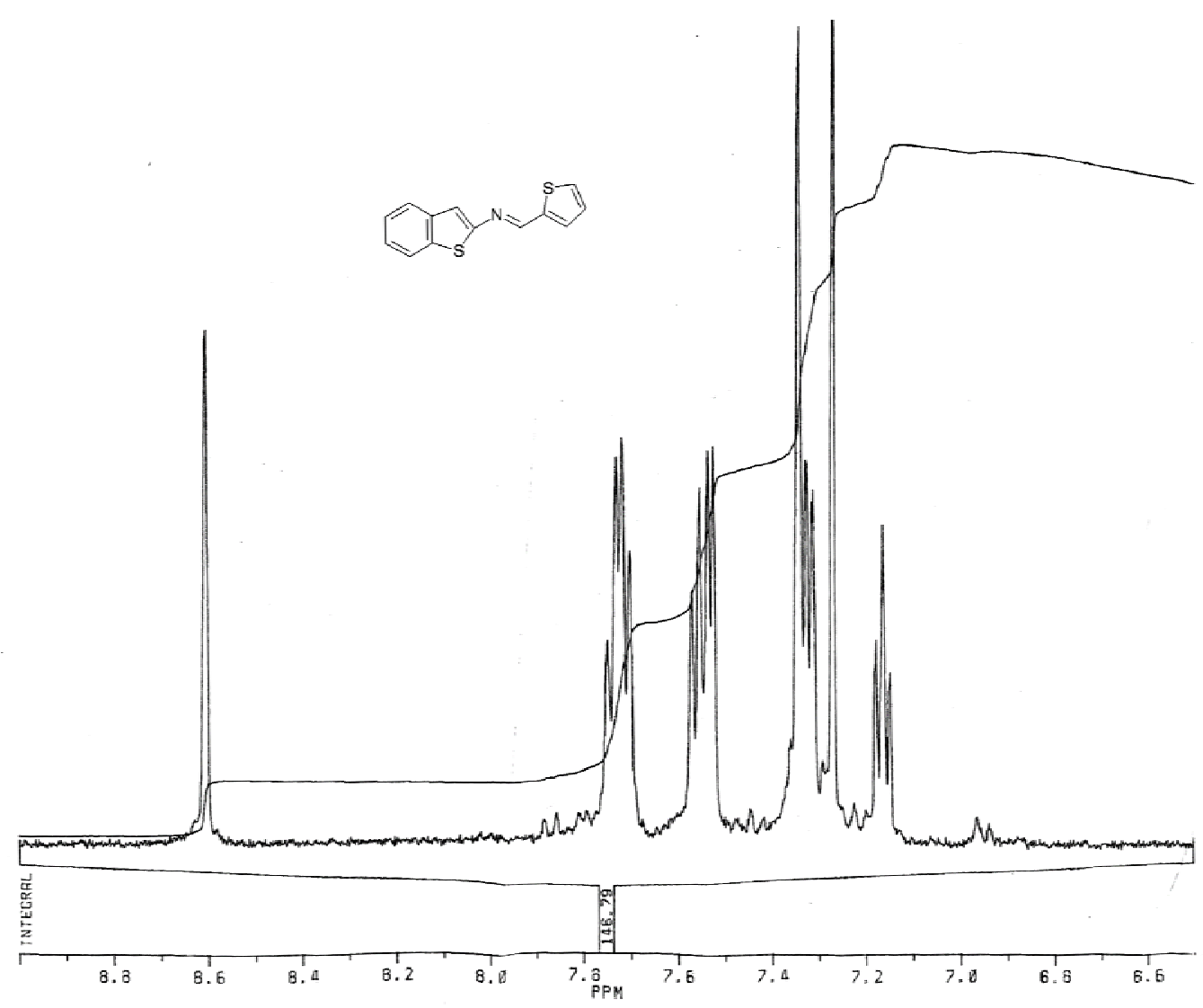




\section{Compound $3 b-{ }^{13} \mathrm{C}$ NMR spectrum}

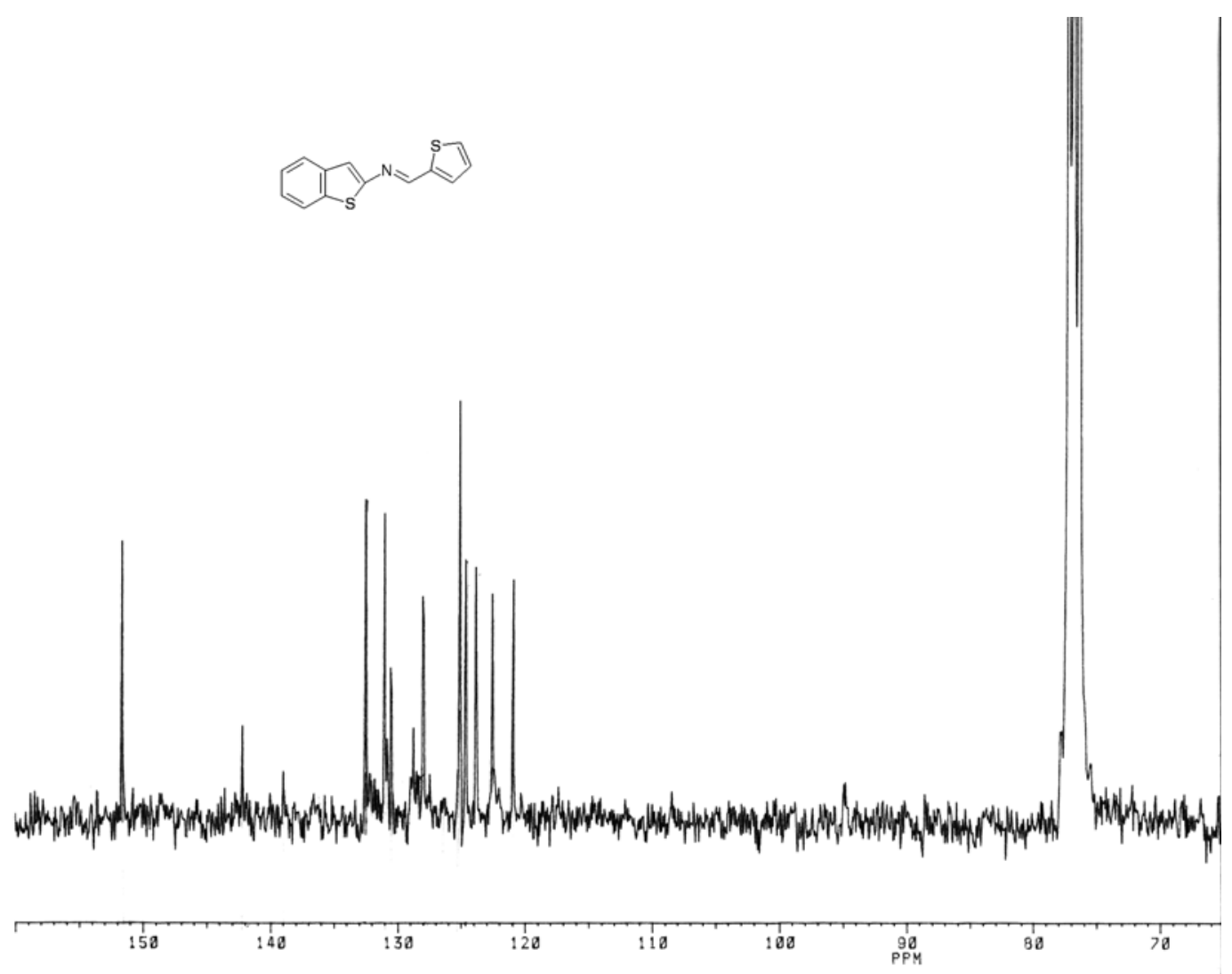




\section{Compound $5 a-{ }^{1} \mathrm{H}$ NMR spectrum}

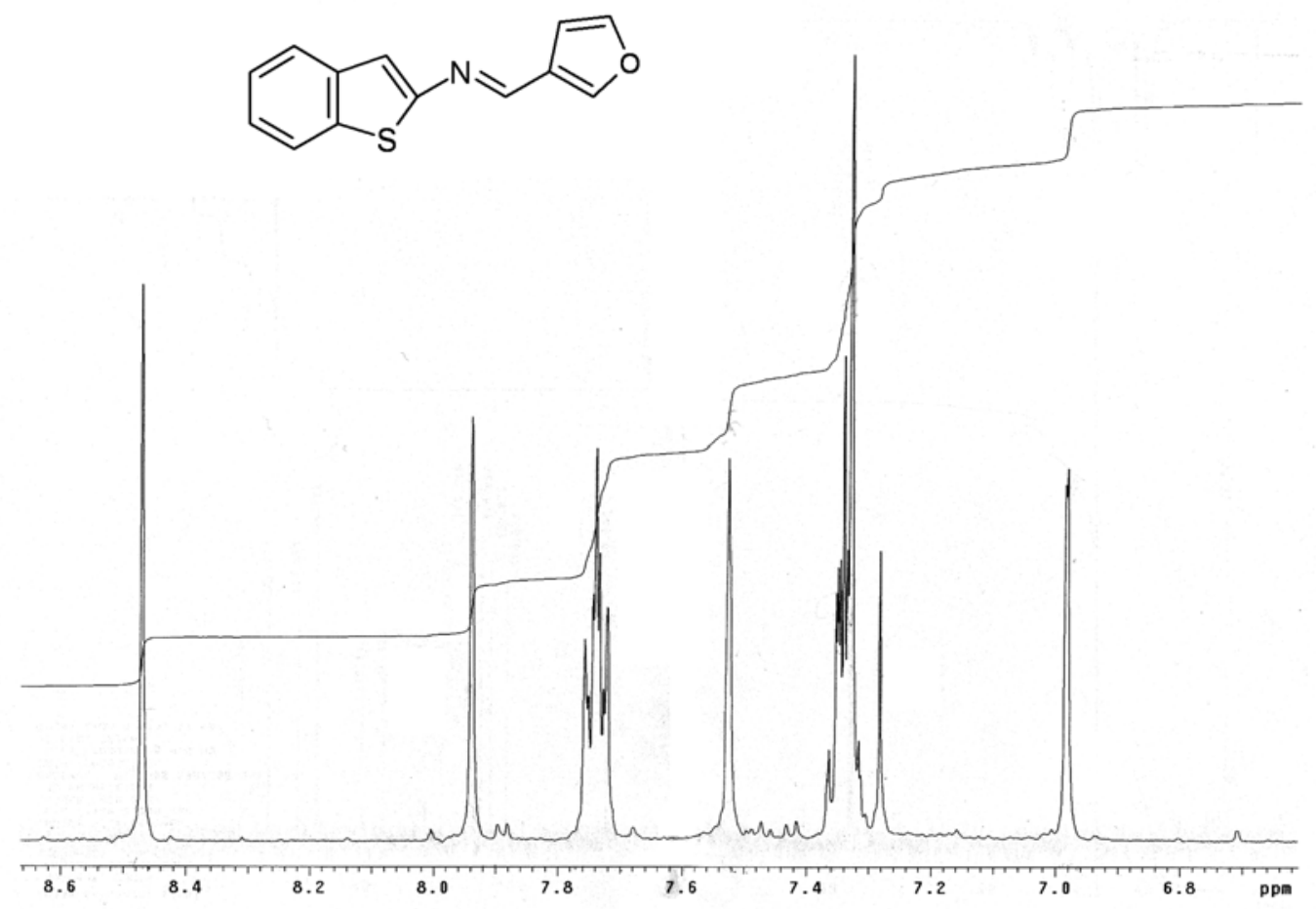




\section{Compound 5a $-{ }^{13} \mathrm{C}$ NMR spectrum}<smiles>C(=N/c1cc2ccccc2s1)\c1ccoc1</smiles>

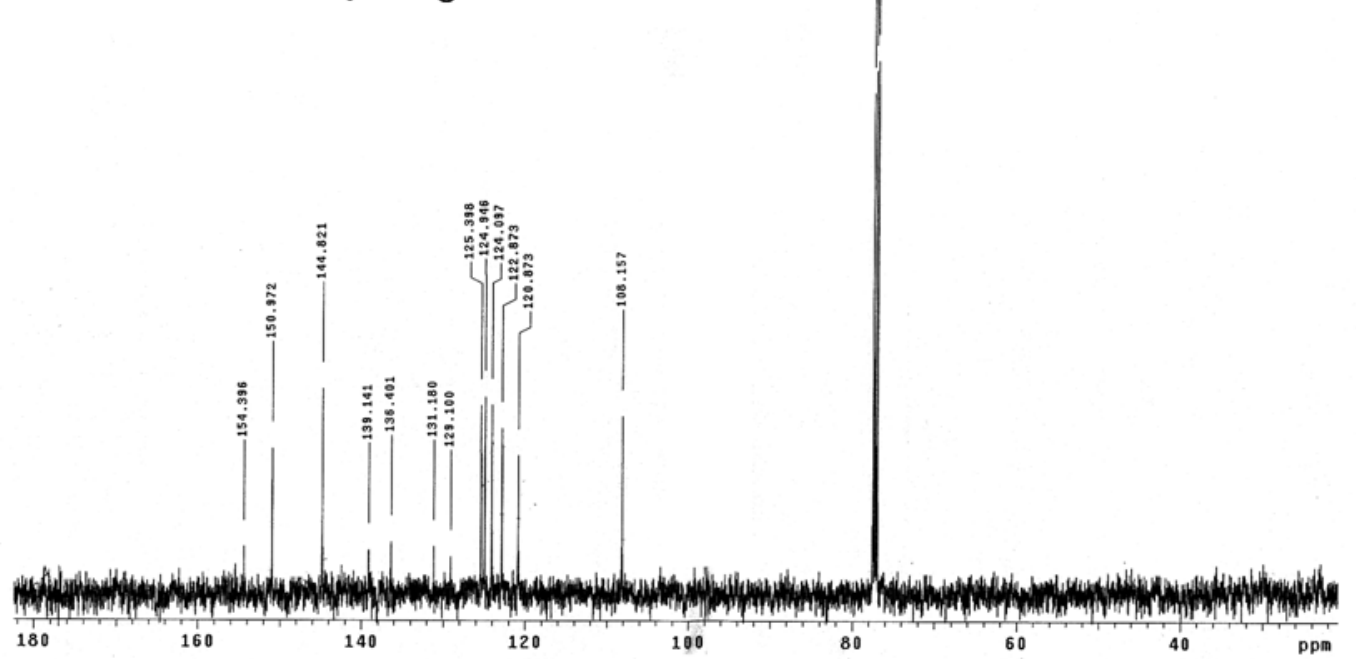




\section{Compound $5 b-{ }^{1} \mathrm{H}$ NMR spectrum}

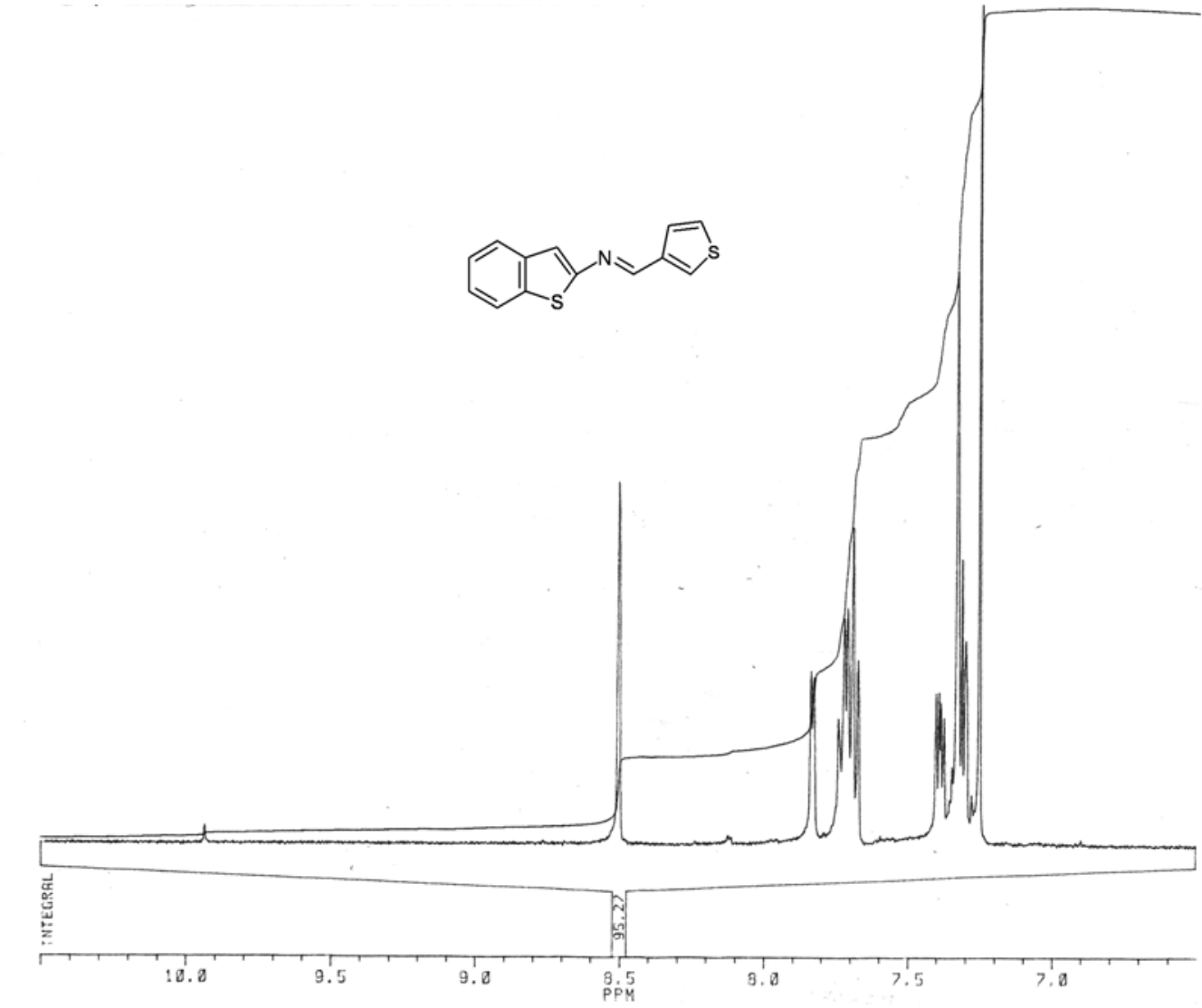




\section{Compound 5b $-{ }^{13} \mathrm{C}$ NMR spectrum}

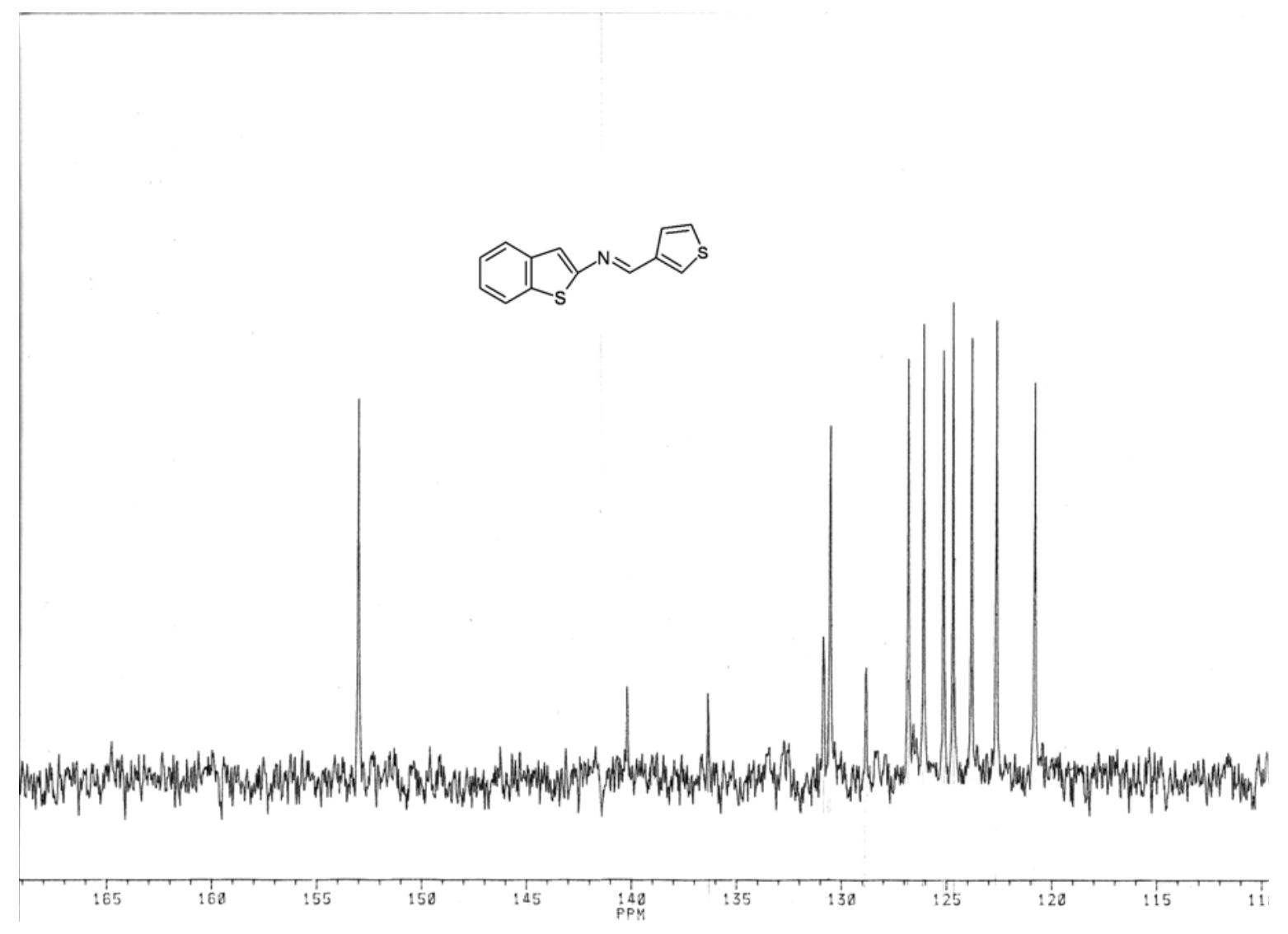




\section{Compound $3 c-{ }^{1} \mathrm{H}$ NMR spectrum}

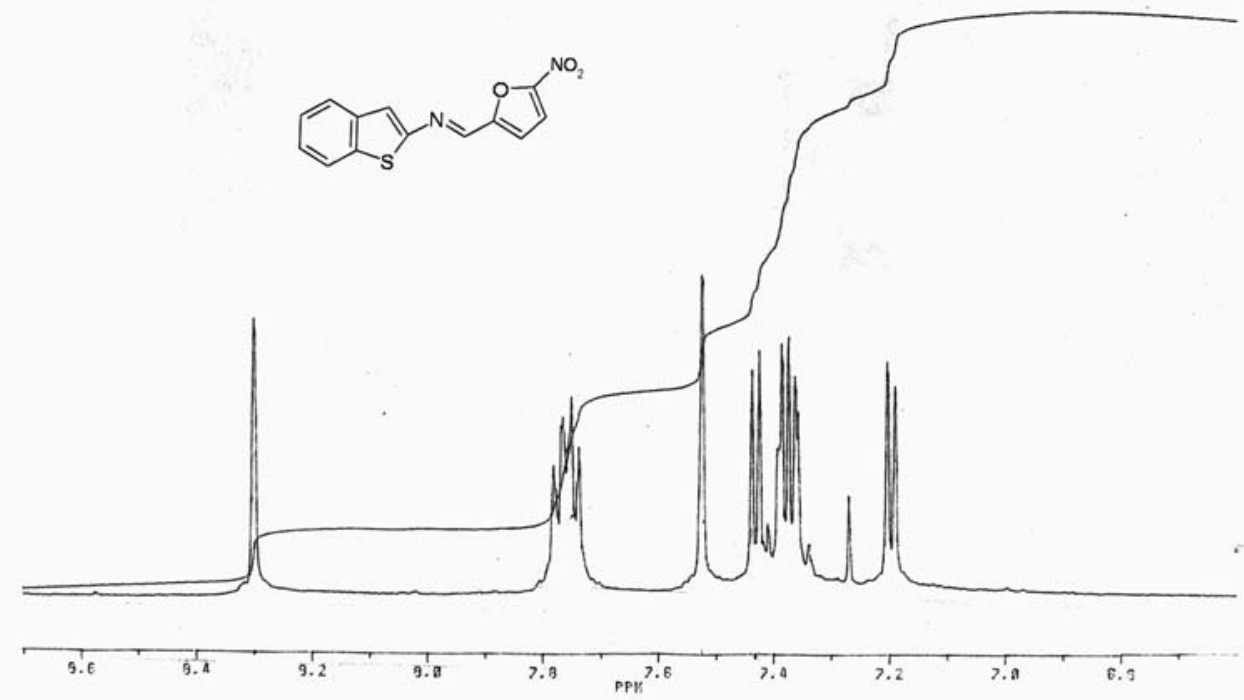




\section{Compound $3 c-{ }^{13} \mathrm{C}$ NMR spectrum}
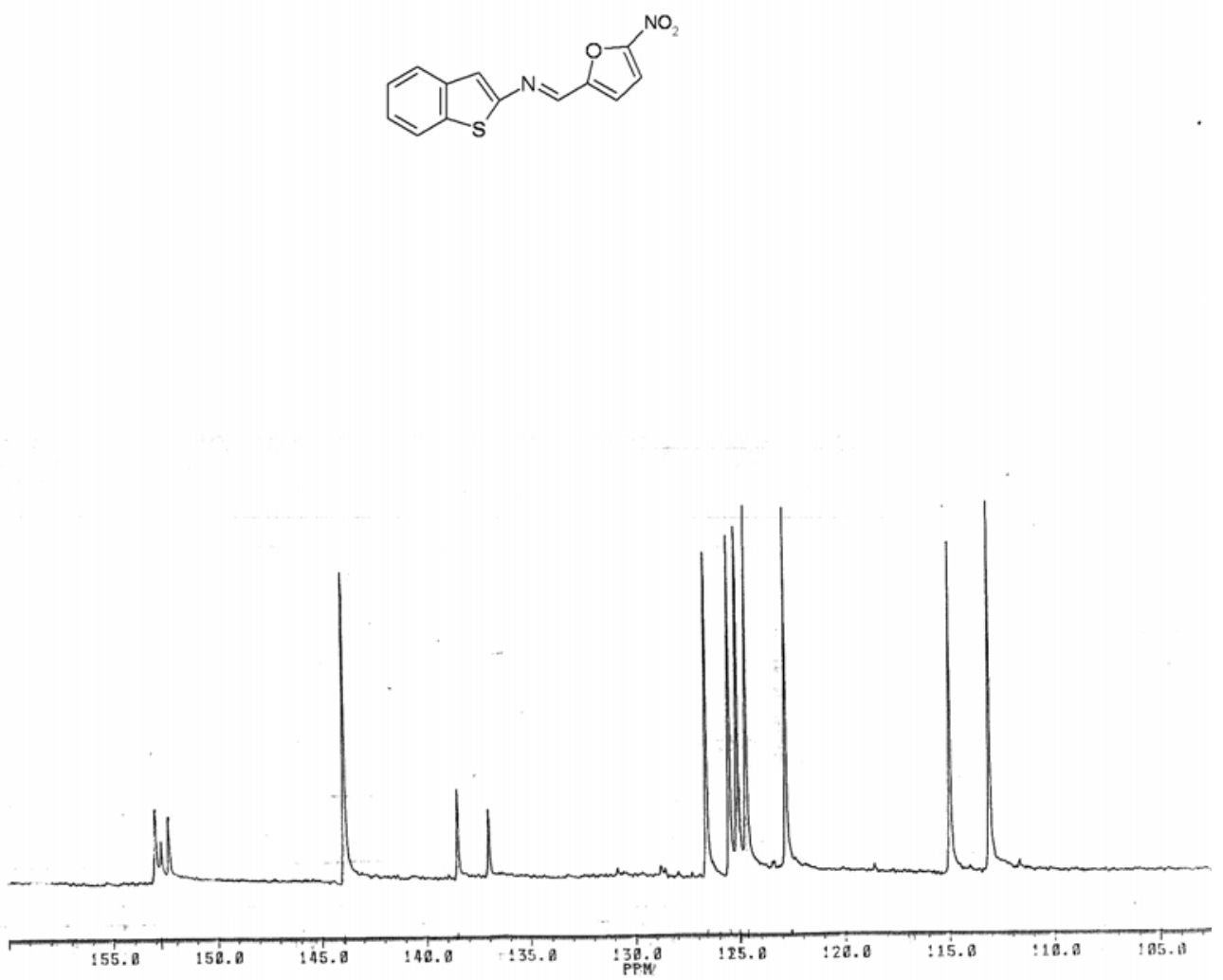


\section{Compound 3d - ${ }^{1} \mathrm{H}$ NMR spectrum}
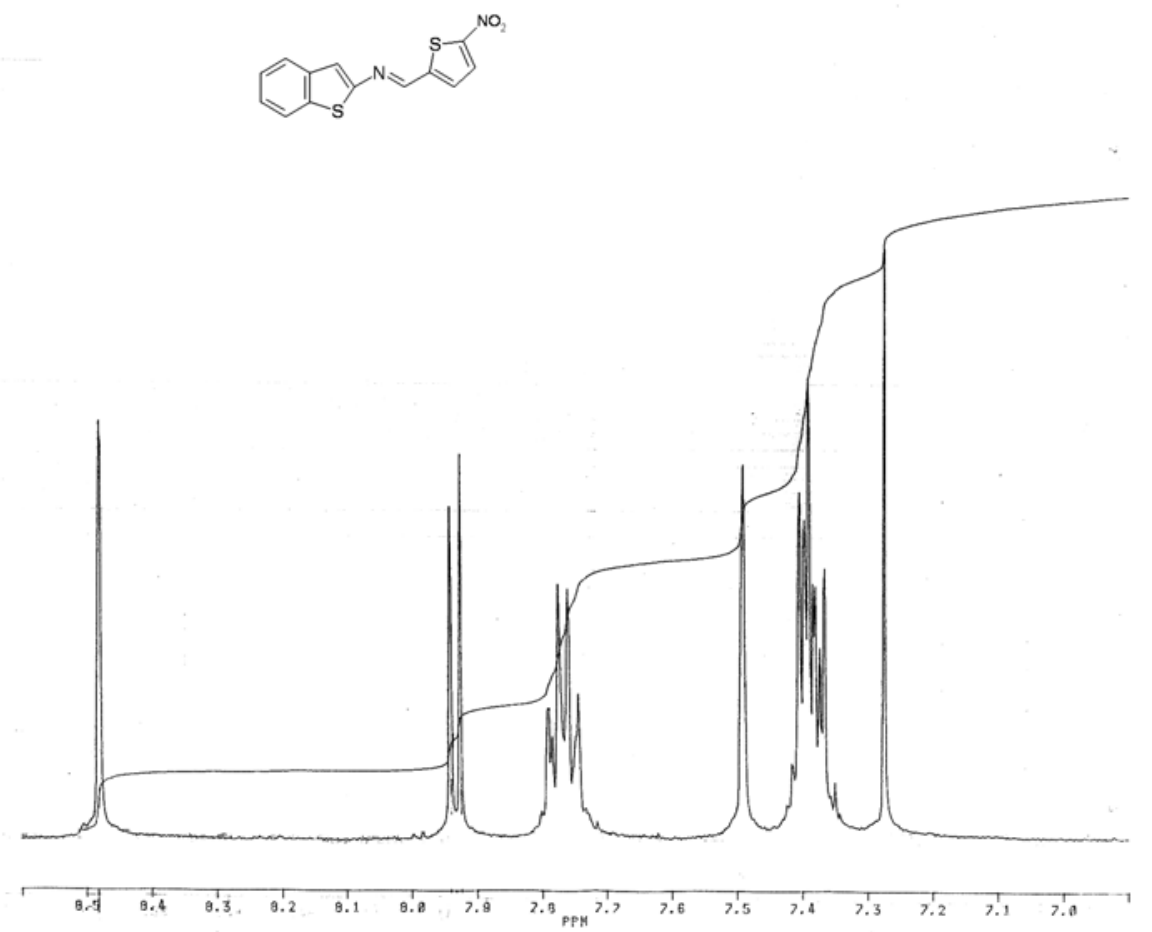


\section{Compound 3d $-{ }^{13} \mathrm{C}$ NMR spectrum}

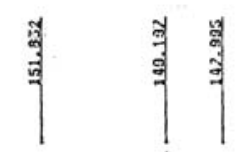
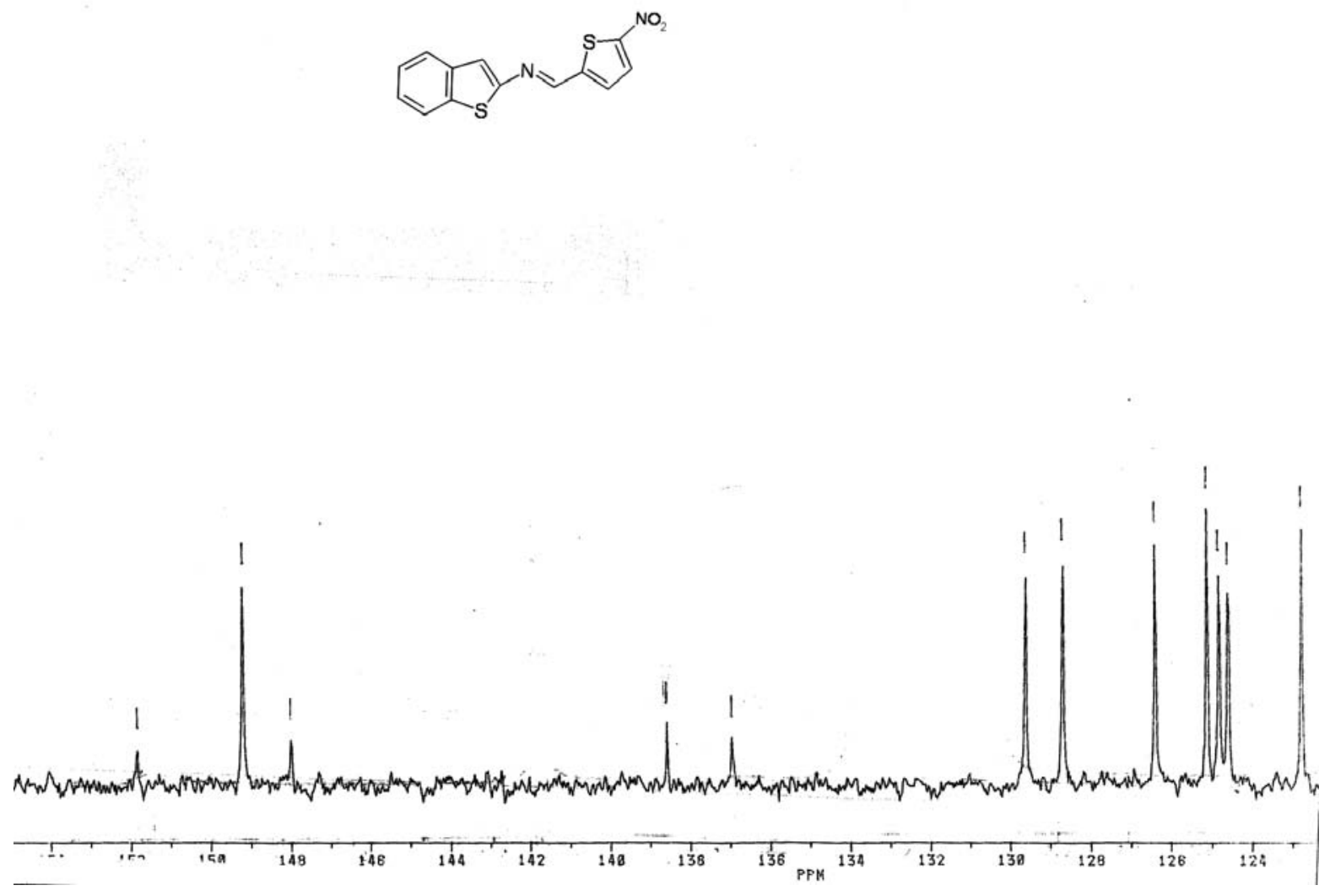


\section{Compound 6a $-{ }^{1} \mathrm{H}$ NMR spectrum}

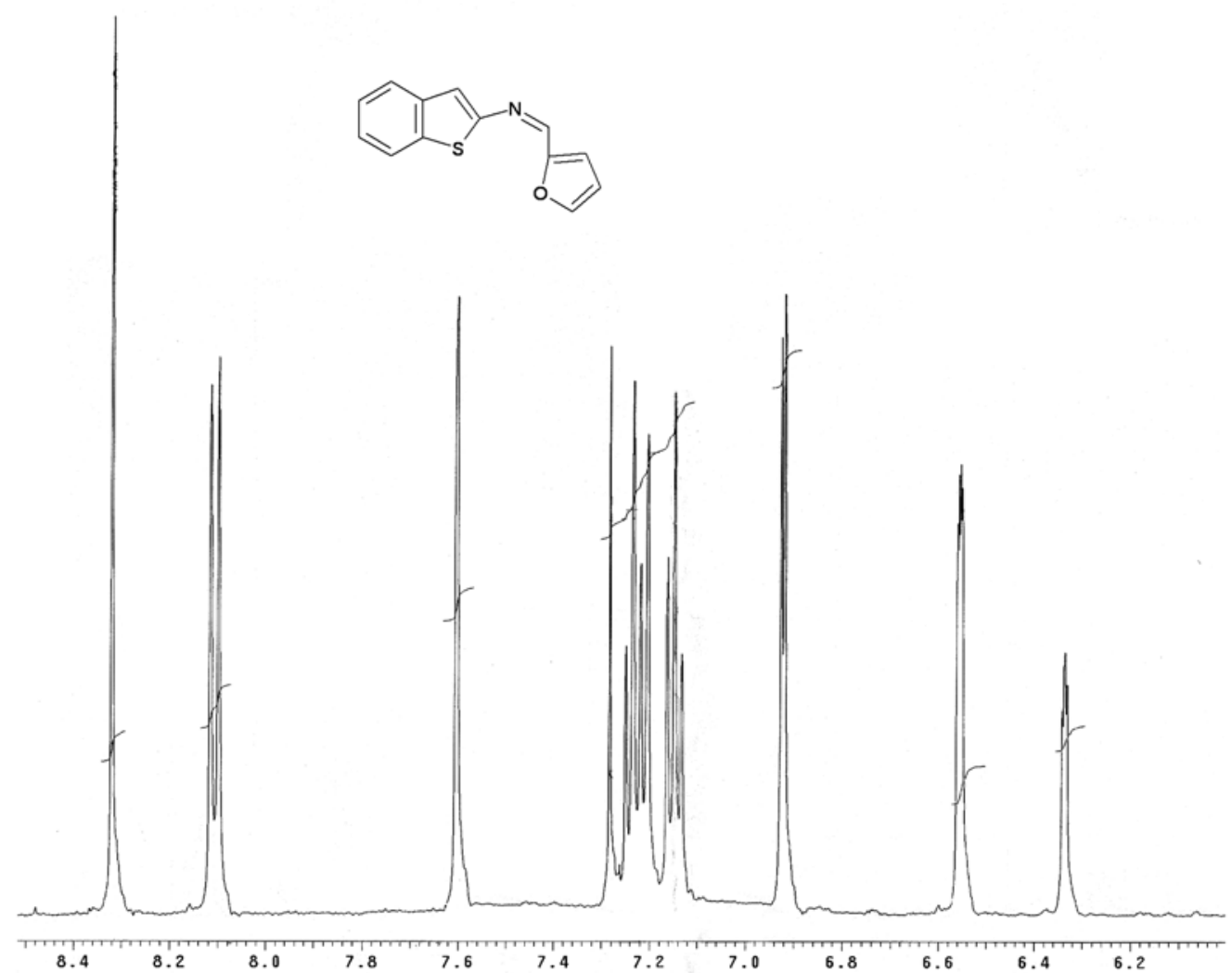




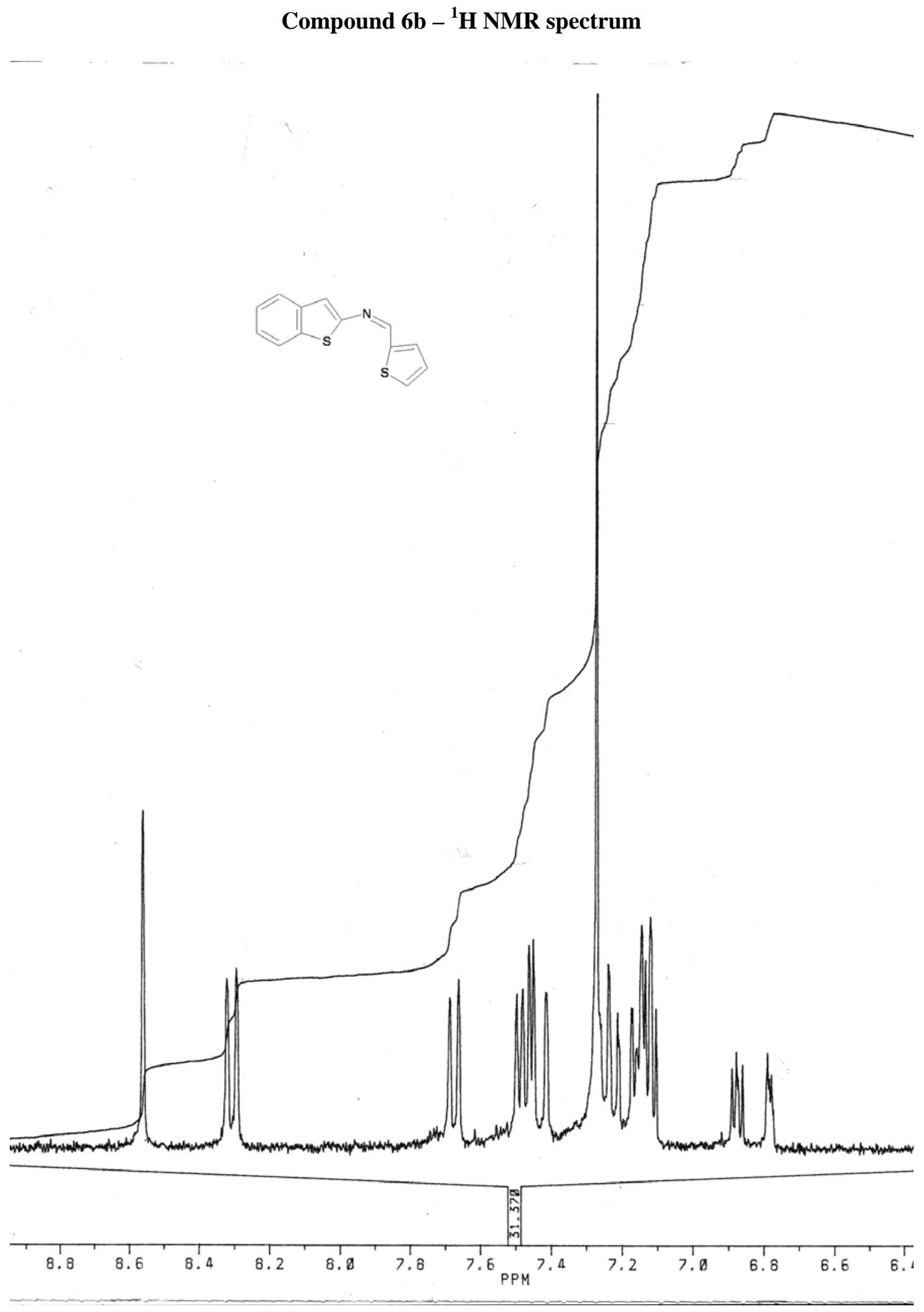

S16 


\section{Compound 6b $-{ }^{13} \mathrm{C}$ NMR spectrum}

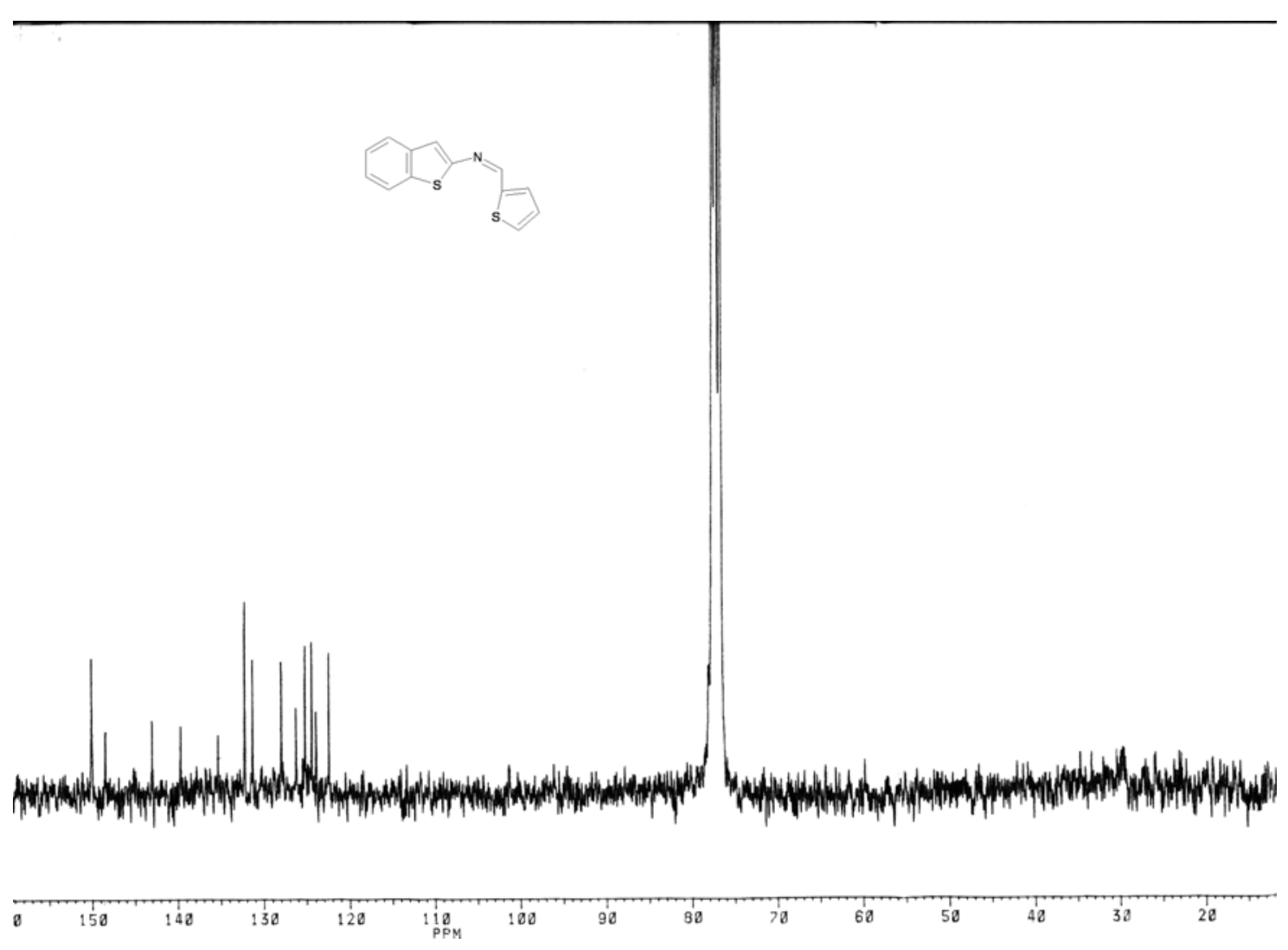




\section{Compound 7a $-{ }^{1} \mathrm{H}$ NMR spectrum}

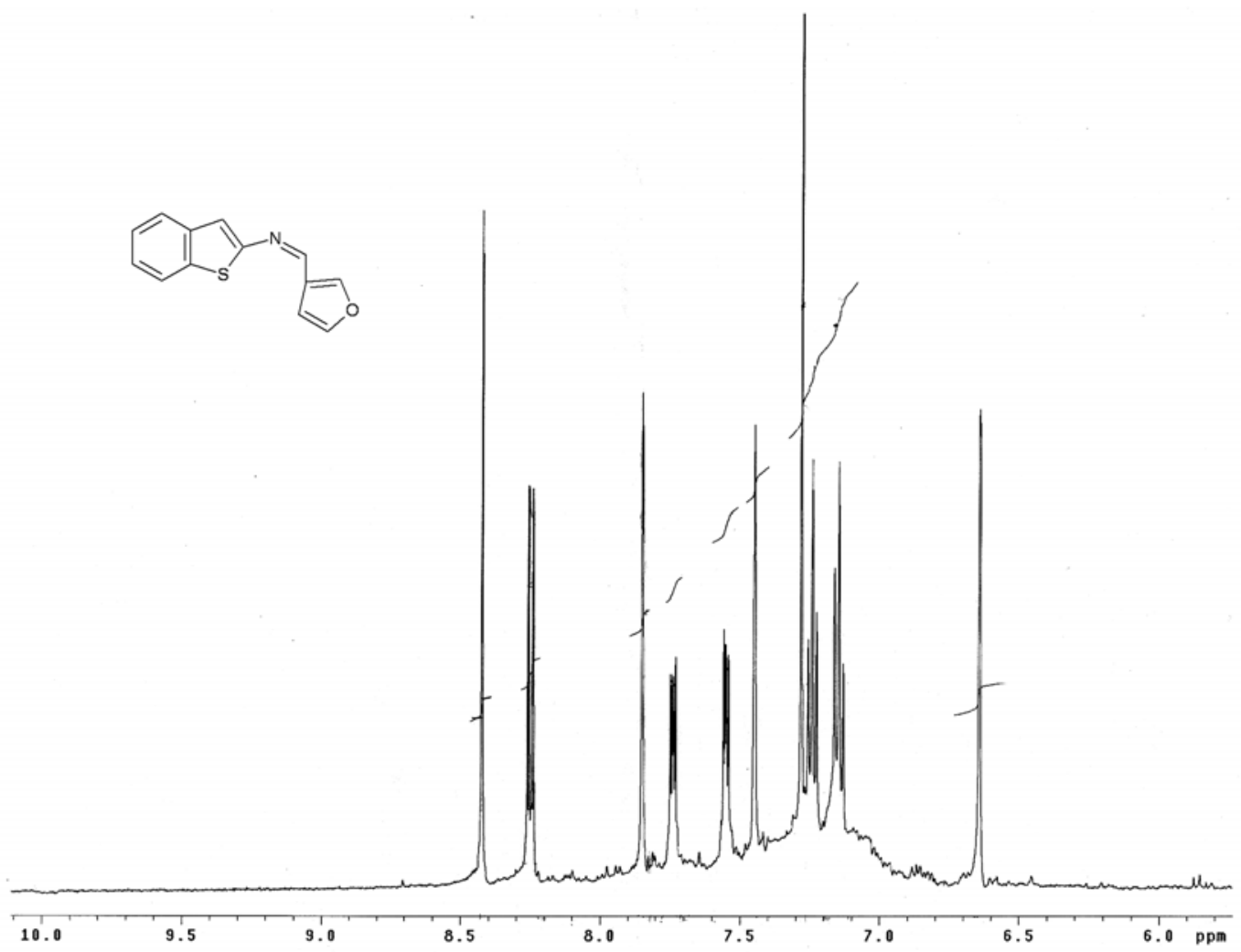




\section{Compound $7 b-{ }^{1} H$ NMR spectrum}
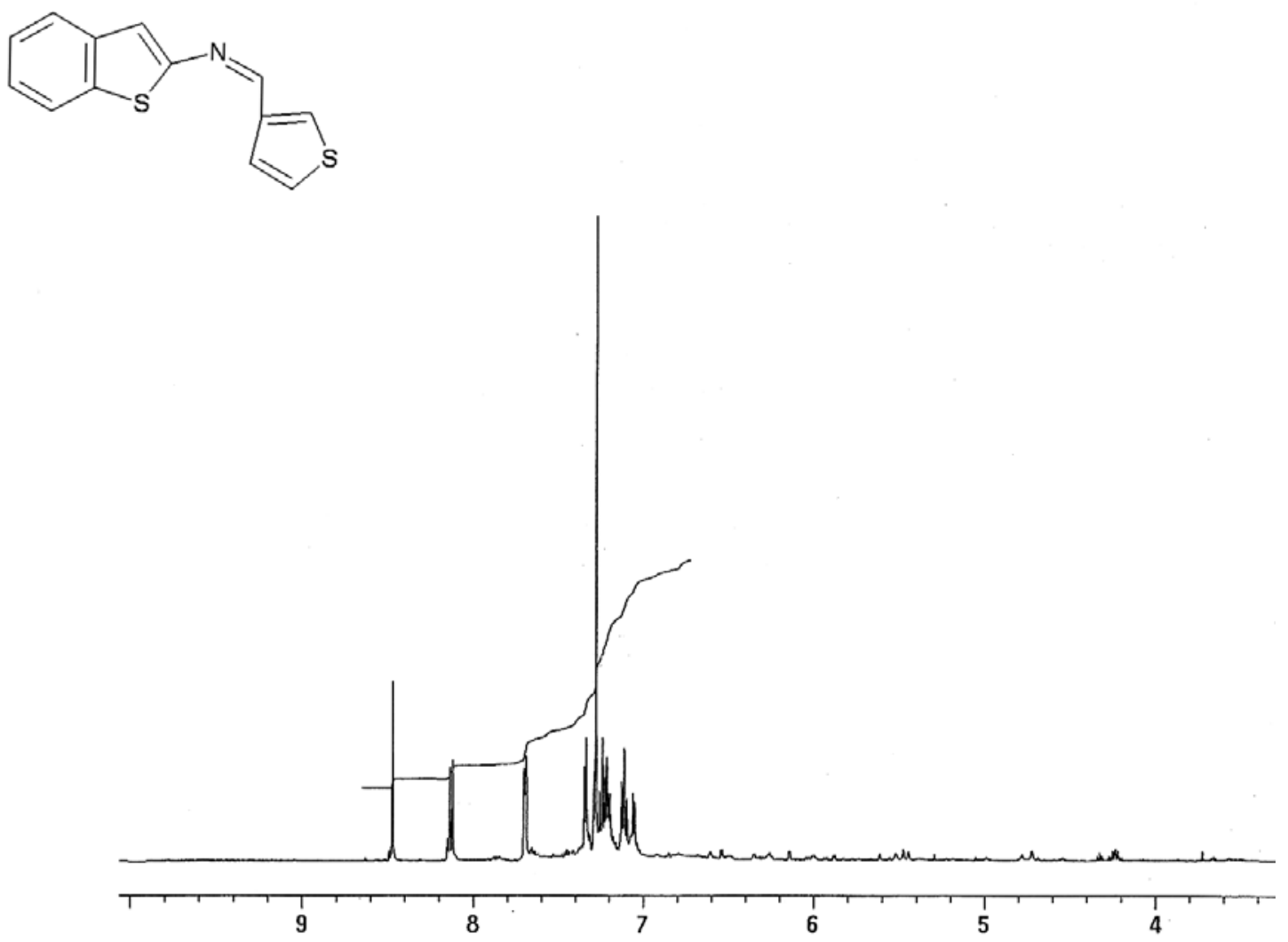


\section{Compound $7 \mathrm{~b}-{ }^{13} \mathrm{C}$ NMR spectrum}

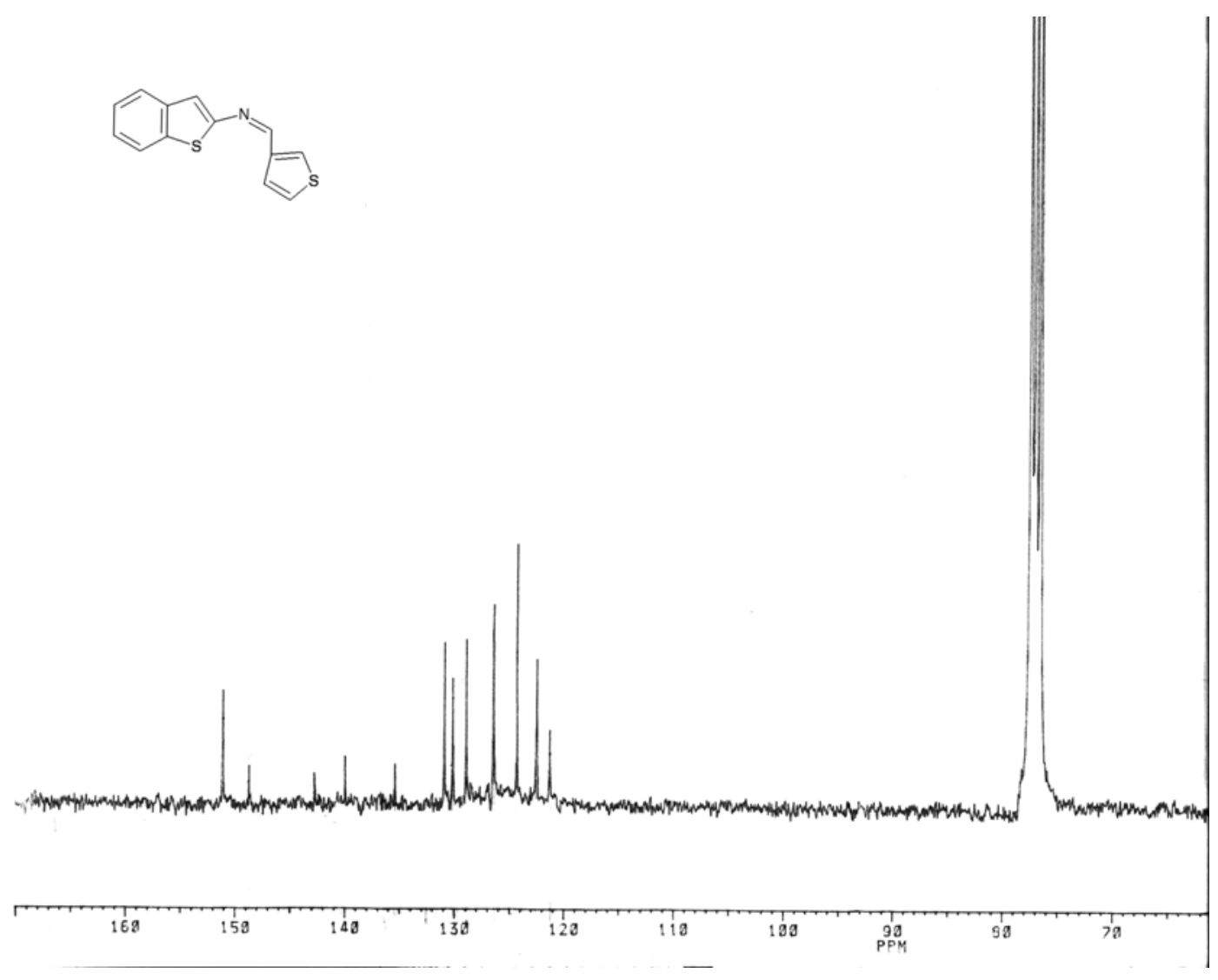




\section{Compound $8-{ }^{1} \mathrm{H}$ NMR spectrum}

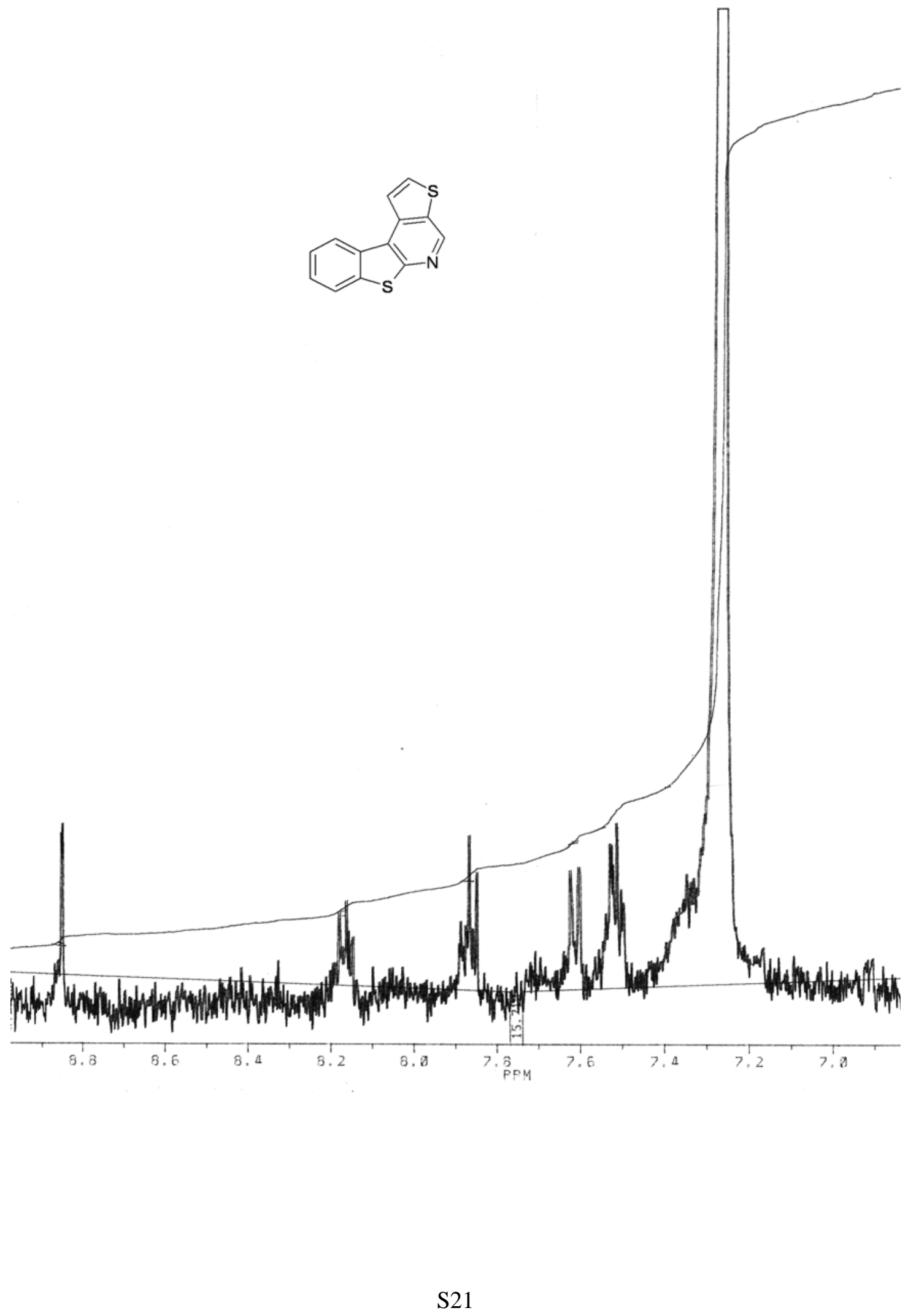




\section{Compound $9-{ }^{1} \mathrm{H}$ NMR spectrum}

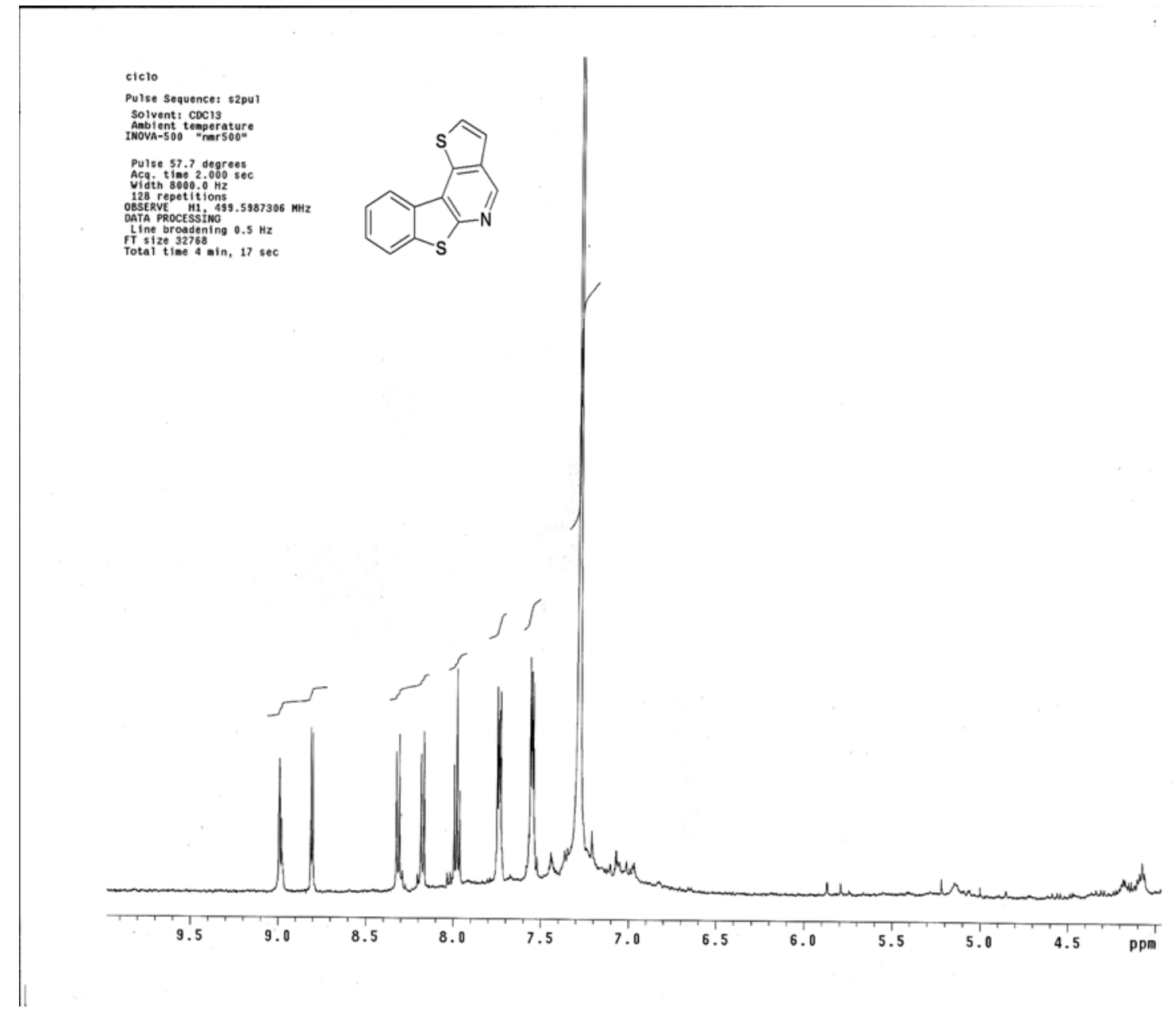




\section{Compound $9-{ }^{13} \mathrm{C}$ NMR spectrum}

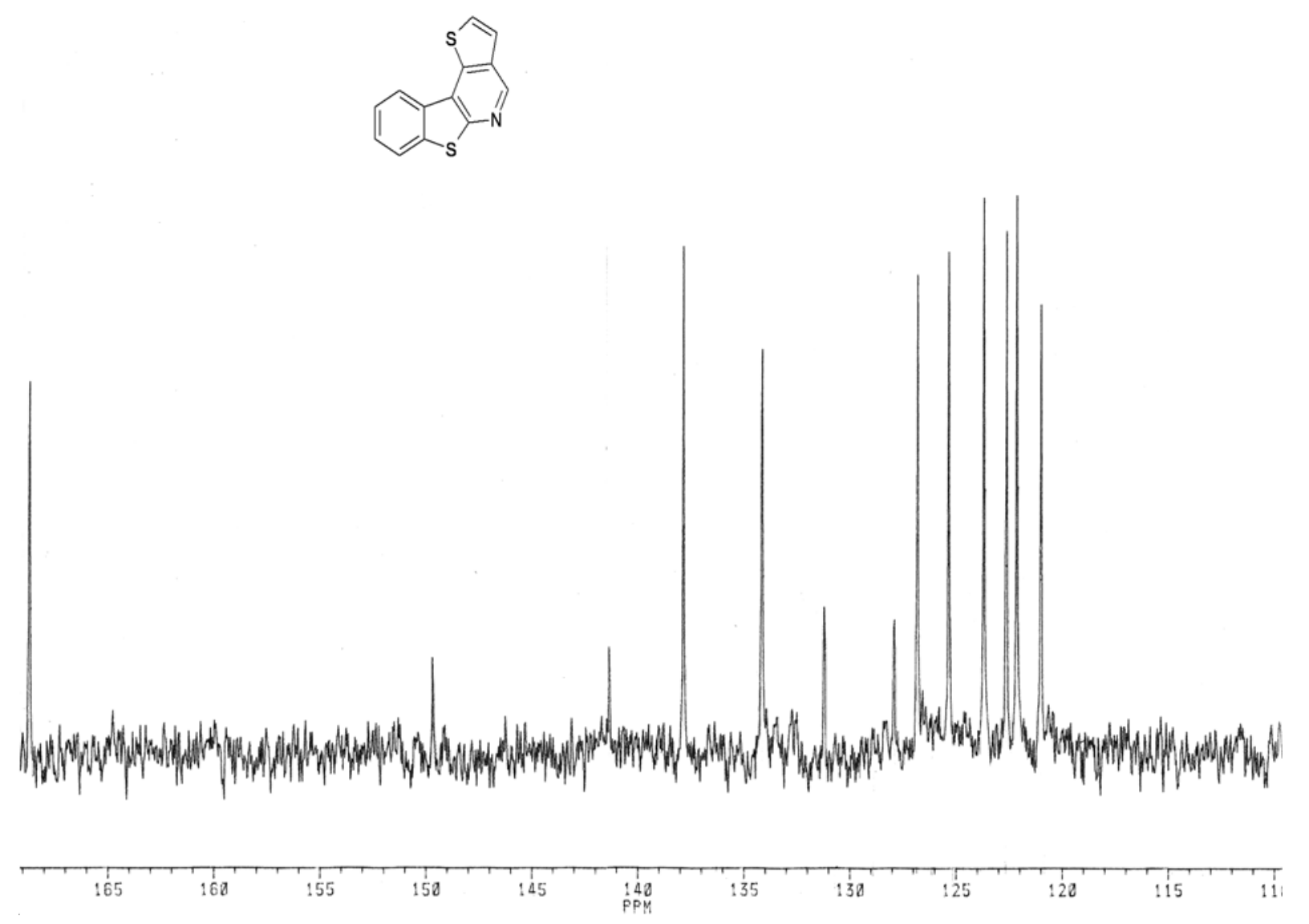




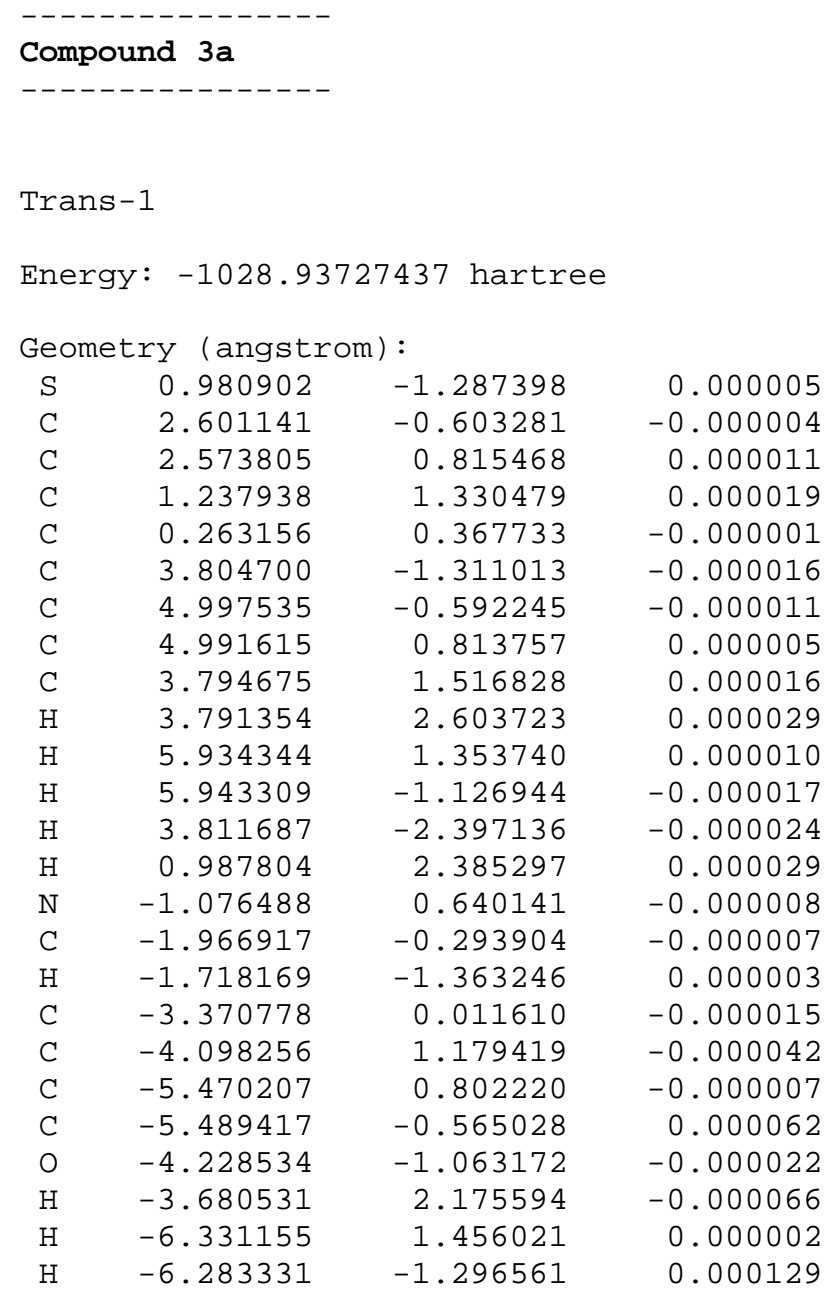




$\begin{array}{rrr}\text { Frequencies }(\mathrm{cm}-1): & \\ 32.1995 & 45.4936 & 59.5952 \\ 119.1361 & 173.2256 & 183.3180 \\ 208.9377 & 249.5395 & 305.9798 \\ 359.6438 & 361.2164 & 444.1968 \\ 471.9550 & 491.1429 & 538.3744 \\ 539.2606 & 571.8329 & 607.1889 \\ 651.4438 & 671.6002 & 673.2108 \\ 716.7387 & 733.6532 & 757.0719 \\ 761.0205 & 809.0346 & 828.7337 \\ 847.1515 & 875.6137 & 884.6017 \\ 888.0321 & 900.4599 & 939.0567 \\ 963.9542 & 978.6204 & 979.3812 \\ 1043.8333 & 1049.4041 & 1078.1851 \\ 1125.5576 & 1156.6395 & 1163.5253 \\ 1190.4084 & 1195.7412 & 1215.3151 \\ 1245.4263 & 1285.1296 & 1285.9497 \\ 1341.6865 & 1373.7499 & 1403.7823 \\ 1449.6935 & 1480.5814 & 1506.1635 \\ 1516.0709 & 1577.6139 & 1615.9603 \\ 1635.4763 & 1647.2357 & 1675.3452 \\ 3066.9016 & 3185.6134 & 3194.0413 \\ 3204.2105 & 3213.4712 & 3236.2791 \\ 3270.2893 & 3290.6025 & 3303.4967\end{array}$

Trans-2

Energy: -1028.93579293 hartree

Geometry (angstrom):

\begin{tabular}{|c|c|c|c|}
\hline 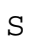 & -1.010376 & -1.333793 & $-0.000 \odot 22$ \\
\hline C & -2.608453 & -0.599415 & 0.000013 \\
\hline & -2.536694 & 0.817821 & $-\odot .000018$ \\
\hline & -1.185567 & 1.291320 & $-\odot .0 \odot \odot \odot 46$ \\
\hline$C$ & -0.240529 & $\odot .299696$ & -0.000016 \\
\hline & -3.833622 & -1.269062 & $\odot .00 \odot \odot 47$ \\
\hline & -5.003374 & $-\odot .513299$ & $\odot .00 \odot 051$ \\
\hline & -4.953484 & 0.891809 & 0.000021 \\
\hline & -3.735046 & 1.556977 & $-\odot .0 \odot \odot \odot 14$ \\
\hline & -3.697510 & 2.643217 & -0.000037 \\
\hline & -5.878837 & 1.461061 & $\odot .000023$ \\
\hline & -5.965430 & -1.018149 & $\odot . \odot \odot \odot \odot 74$ \\
\hline & -3.874689 & -2.354449 & $\odot .0 \odot \odot \odot 63$ \\
\hline & -0.901940 & 2.337588 & $-\odot .00 \odot \odot 66$ \\
\hline & 1.106207 & 0.532899 & -0.000013 \\
\hline & 1.967244 & $-\odot .427069$ & $-\odot .00 \odot \odot \odot 4$ \\
\hline & 1.677231 & -1.486799 & $-\odot .0 \odot \odot \odot \odot 6$ \\
\hline & 3.386130 & $-\odot .205922$ & $-\odot .0 \odot \odot \odot 2 \odot$ \\
\hline C & 4.434809 & -1.099421 & $-\odot .0 \odot \odot \odot 67$ \\
\hline & 5.630072 & $-\odot .325758$ & $\odot .00 \odot \odot 53$ \\
\hline & 5.228107 & $\odot .980250$ & ๑. .00๑124 \\
\hline & 3.875863 & 1.073141 & $-0.00 \odot \odot 76$ \\
\hline & 4.349533 & -2.177539 & $-\odot .0 \odot \odot 101$ \\
\hline & 6.648908 & -0.686601 & 0.000122 \\
\hline & 5.760833 & 1.919386 & 0.000246 \\
\hline
\end{tabular}


45.3774

165.4785

258.1831

363.4367

491.0132

572.4028

669.5379

733.8135

818.0982

876.3790

900.5325

956.8119

1052.5709

1158.3624

1200.7164

1285.5249

1372.8426

1477.7293

1577.5894

1648.7555

3185.5090

3213.2649

3278.1252
60.4842

183.5327

322.2788

444.3350

539.2983

605.8054

684.6427

761.1445

819.0198

878.9376

939.0278

979.3478

1077.9281

1169.9535

1215.3827

1319.3310

1395.5086

1504.0185

1614.4765

1686.3073

3193.9857

3237.2353

3302.7140

Trans - 3

Energy: -1028.93329770 hartree

Geometry (angstrom) :

\begin{tabular}{|c|c|c|c|}
\hline S & -1.324448 & -1.655090 & -0.145384 \\
\hline C & -2.734653 & -0.611256 & -0.079420 \\
\hline C & -2.365109 & ๑. 740724 & ๑. 151963 \\
\hline C & -0.944434 & 0.905760 & 0.252334 \\
\hline C & -0.247459 & -0.265612 & ○. . 098496 \\
\hline C & -4.072047 & $-\odot .989513$ & -0.223652 \\
\hline C & -5.054527 & $-\odot .008904$ & -0.125989 \\
\hline C & -4.709081 & 1.334170 & ๑. 109844 \\
\hline C & -3.380918 & 1.711540 & 0.249503 \\
\hline $\mathrm{H}$ & -3.118015 & 2.750968 & ○. 428790 \\
\hline $\mathrm{H}$ & -5.492393 & 2.083655 & 0.182505 \\
\hline $\mathrm{H}$ & -6.099733 & -0.285998 & -0.231371 \\
\hline $\mathrm{H}$ & -4.340013 & -2.026697 & -0.403793 \\
\hline $\mathrm{H}$ & -0.465341 & 1.853779 & ○. 472984 \\
\hline $\mathrm{N}$ & 1.101590 & -0.537141 & 0.171691 \\
\hline C & 1.954394 & ๑. 360999 & -0.187421 \\
\hline $\mathrm{H}$ & 1.658443 & 1.321583 & -0.628329 \\
\hline C & 3.369356 & $\odot .152134$ & $-\odot .039554$ \\
\hline C & 4.139223 & -0.867880 & 0.468626 \\
\hline C & 5.495598 & -0.466264 & 0.316527 \\
\hline C & 5.463348 & ๑.768905 & -0.269433 \\
\hline 0 & 4.185872 & 1.161521 & -0.493779 \\
\hline $\mathrm{H}$ & 3.758466 & -1.785077 & ๑. 893756 \\
\hline $\mathrm{H}$ & 6.380048 & -1.017157 & 0.604356 \\
\hline $\mathrm{H}$ & 6.229446 & 1.465382 & -0.575308 \\
\hline
\end{tabular}




$\begin{array}{rrr}\text { Frequencies }(\mathrm{cm}-1): & \\ 39.3510 & 45.4986 & 56.9208 \\ 125.1678 & 163.4277 & 196.2814 \\ 198.8592 & 256.1396 & 295.5887 \\ 341.0000 & 378.2344 & 441.3510 \\ 456.5584 & 493.4721 & 542.0317 \\ 546.3927 & 580.6360 & 607.7720 \\ 654.0901 & 672.3778 & 705.9352 \\ 720.1997 & 733.7991 & 757.2907 \\ 759.8703 & 815.7622 & 832.5726 \\ 841.1105 & 869.6120 & 890.7731 \\ 892.0619 & 90 \odot .5473 & 936.9852 \\ 977.6409 & 979.6328 & 992.6400 \\ 1043.8346 & 1049.2498 & 1079.0878 \\ 1126.1320 & 1150.6118 & 1164.0761 \\ 1191.4987 & 1194.5769 & 1220.6772 \\ 1241.9722 & 1283.7197 & 1288.5030 \\ 1348.9923 & 1379.2776 & 1398.3060 \\ 1447.9191 & 1475.6526 & 1505.3114 \\ 1517.9456 & 1560.4284 & 1613.5219 \\ 1630.7145 & 1648.5367 & 1669.1148 \\ 3071.7927 & 3183.4267 & 3192.2032 \\ 3201.9805 & 3212.1693 & 3221.3975 \\ 3271.0364 & 3290.3529 & 3303.2329\end{array}$

Trans - 4

Energy: -1028.93160875 hartree

Geometry (angstrom) :

\begin{tabular}{|c|c|c|c|}
\hline $\mathrm{S}$ & 1.272158 & -1.625249 & 0.157845 \\
\hline C & 2.708972 & -0.619238 & ๑. .078479 \\
\hline C & 2.373993 & ๑. 742018 & -0.150989 \\
\hline C & ๑. 957070 & 0.944847 & -0.236814 \\
\hline C & 0.230652 & -0.207492 & -0.075357 \\
\hline C & 4.037040 & -1.033101 & ๑. 210885 \\
\hline C & 5.044747 & -0.079555 & 0.102738 \\
\hline C & 4.733486 & 1.272048 & -0.131625 \\
\hline C & 3.414617 & 1.684902 & -0.259327 \\
\hline $\mathrm{H}$ & 3.178278 & 2.730892 & -0.437667 \\
\hline $\mathrm{H}$ & 5.535995 & $2 . \odot \odot \odot \odot 74$ & -0.212767 \\
\hline $\mathrm{H}$ & 6.083051 & -0.384734 & ๑. 198415 \\
\hline $\mathrm{H}$ & 4.278591 & -2.077017 & 0. 389595 \\
\hline $\mathrm{H}$ & 0.501069 & 1.904735 & -0.455315 \\
\hline $\mathrm{N}$ & -1.125991 & -0.441670 & -0.138310 \\
\hline C & -1.946049 & $\odot .472464$ & ๑. 251696 \\
\hline $\mathrm{H}$ & -1.606301 & 1.411369 & 0.709526 \\
\hline C & -3.373353 & 0.346679 & 0.137858 \\
\hline C & -4.383774 & 1.198895 & 0.524696 \\
\hline C & -5.608984 & $\odot .567736$ & ๑.167895 \\
\hline C & -5.260218 & -0.620706 & -0.409648 \\
\hline 0 & -3.914396 & -0.771556 & -0.438120 \\
\hline $\mathrm{H}$ & -4.254959 & 2.158239 & 1.007144 \\
\hline $\mathrm{H}$ & -6.611791 & ๑. 941665 & ๑. 318461 \\
\hline $\mathrm{H}$ & -5.830547 & -1.434095 & -0.832696 \\
\hline
\end{tabular}




$\begin{array}{rrr}\text { Frequencies }(\mathrm{cm}-1): & \\ 38.4378 & 44.7319 & 57.2340 \\ 118.6392 & 156.3403 & 195.1283 \\ 199.7298 & 266.4805 & 308.9969 \\ 346.0686 & 381.5424 & 441.7595 \\ 456.6717 & 493.5772 & 542.9448 \\ 547.0025 & 582.0523 & 606.3372 \\ 653.8824 & 683.2469 & 707.3680 \\ 721.1951 & 733.8840 & 757.1540 \\ 765.9243 & 816.3505 & 822.8824 \\ 846.5342 & 869.3904 & 880.4170 \\ 892.5765 & 90 \odot .5174 & 936.6700 \\ 956.0276 & 977.2365 & 986.8477 \\ 1048.9132 & 1053.1855 & 1079.2444 \\ 1118.7973 & 1155.8312 & 1168.3802 \\ 1191.3856 & 1201.9062 & 1219.7934 \\ 1259.3327 & 1285.3885 & 1324.8964 \\ 1348.4851 & 1378.1606 & 1387.9227 \\ 1445.9912 & 1472.0276 & 1503.9294 \\ 1520.9297 & 1560.5910 & 1611.6253 \\ 1618.2576 & 1649.7329 & 1680.3903 \\ 3058.5950 & 3182.9891 & 3191.8078 \\ 3201.6500 & 3211.9246 & 3220.3704 \\ 3266.3715 & 3278.4882 & 3303.0424\end{array}$

\section{Compound $6 a$}

Cis-1

Energy: -1028.92588006 hartree

Geometry (angstrom) :

\begin{tabular}{|c|c|c|c|}
\hline 5 & -1.233161 & -1.811125 & -0.587692 \\
\hline C & -2.346211 & $-\odot .477209$ & $-\odot .314686$ \\
\hline c & -1.793844 & 0.477689 & 0.578011 \\
\hline $\mathrm{C}$ & -0.464790 & $\odot .127301$ & ๑. 996558 \\
\hline C & -0.013060 & -1.045131 & 0.452849 \\
\hline C & -3.615801 & -0.311487 & $-\odot .872262$ \\
\hline C & -4.354764 & 0.814759 & $-\odot .519570$ \\
\hline C & -3.829936 & 1.766617 & 0.371646 \\
\hline C & -2.563205 & 1.606270 & ๑. 918181 \\
\hline $\mathrm{H}$ & -2.160566 & 2.347283 & 1.604217 \\
\hline $\mathrm{H}$ & -4.422809 & 2.638428 & 0.634620 \\
\hline $\mathrm{H}$ & -5.348224 & $\odot .955333$ & -0.936170 \\
\hline $\mathrm{H}$ & -4.022556 & -1.048627 & -1.558774 \\
\hline$H$ & 0.119169 & 0.710371 & 1.700373 \\
\hline $\mathrm{N}$ & 1.120577 & -1.784452 & ๑.728749 \\
\hline C & 2.325493 & -1.347024 & 0.628328 \\
\hline $\mathrm{H}$ & 3.108985 & -2.035897 & ๑. 953175 \\
\hline C & 2.857625 & -0.103822 & 0.105765 \\
\hline C & 2.378676 & 1.043559 & -0.487765 \\
\hline C & 3.511209 & 1.825366 & -0.848353 \\
\hline C & 4.607055 & 1.113218 & -0.448375 \\
\hline 0 & 4.237615 & $-\odot .052080$ & 0.126816 \\
\hline $\mathrm{H}$ & 1.339205 & 1.289754 & -0.639912 \\
\hline$H$ & 3.511901 & 2.790889 & -1.334298 \\
\hline $\mathrm{H}$ & 5.669831 & 1.297652 & $-\odot .497896$ \\
\hline
\end{tabular}




$\begin{array}{rrr}\text { Frequencies }(\mathrm{cm}-1): & \\ 24.7525 & 50.2431 & 56.3950 \\ 127.5364 & 148.1856 & 199.4353 \\ 214.9662 & 251.5987 & 305.8981 \\ 371.1888 & 426.9557 & 458.3471 \\ 478.5374 & 495.2069 & 544.1667 \\ 565.1470 & 601.4314 & 609.0589 \\ 634.6534 & 670.3749 & 704.3317 \\ 720.7689 & 733.2919 & 757.1453 \\ 768.8589 & 804.3528 & 831.2623 \\ 845.5578 & 868.9236 & 881.4717 \\ 901.4195 & 903.5902 & 934.7836 \\ 965.4653 & 967.1868 & 976.3339 \\ 1048.2406 & 1052.6862 & 1076.8921 \\ 1124.6793 & 1131.2894 & 1161.8739 \\ 1186.2217 & 1190.7347 & 1217.5197 \\ 1219.9953 & 1282.5158 & 1286.3345 \\ 1343.6076 & 1377.1274 & 1420.8657 \\ 1457.7998 & 1485.5467 & 1504.9612 \\ 1509.1718 & 1566.2155 & 1614.9704 \\ 1618.7535 & 1648.8194 & 1698.0416 \\ 3135.6015 & 3182.2957 & 3191.3874 \\ 3200.9763 & 3211.4926 & 3225.0382 \\ 3273.3224 & 3299.8714 & 3305.0354\end{array}$

Cis - 2

Energy: -1028.92484997 hartree

Geometry (angstrom):

$\begin{array}{lrrr}\text { C } & -2.376053 & -\Theta .496425 & -0.237227 \\ \text { C } & -3.694287 & -0.342866 & -0.673832 \\ \text { C } & -4.353969 & 0.849943 & -0.391910 \\ \text { C } & -3.704777 & 1.881865 & 0.309428 \\ \text { C } & -2.391584 & 1.734040 & 0.734083 \\ \text { C } & -1.699298 & 0.538135 & 0.461607 \\ \text { H } & -4.197417 & -1.140078 & -1.213706 \\ \text { H } & -5.382727 & 0.982139 & -0.715413 \\ \text { H } & -4.238204 & 2.805054 & 0.519049 \\ \text { H } & -1.891379 & 2.535814 & 1.271499 \\ \text { C } & -0.345122 & 0.189215 & 0.777263 \\ \text { C } & 0.013426 & -1.061142 & 0.346200 \\ \text { S } & -1.343665 & -1.899711 & -0.446721 \\ \text { N } & 1.131503 & -1.832694 & 0.566237 \\ \text { C } & 2.361536 & -1.457241 & 0.522452 \\ \text { H } & 3.088387 & -2.223504 & 0.806282 \\ \text { C } & 3.019677 & -\Theta .233867 & 0.119855 \\ \text { C } & 4.372031 & 0.021091 & 0.044293 \\ \text { C } & 4.516352 & 1.339456 & -0.471058 \\ \text { C } & 3.246947 & 1.799646 & -0.676464 \\ \text { O } & 2.330029 & 0.864340 & -0.324488 \\ \text { H } & 0.329390 & 0.840254 & 1.319244 \\ \text { H } & 2.845043 & 2.730017 & -1.048740 \\ \text { H } & 5.434850 & 1.875207 & -0.664433 \\ \text { H } & 5.160668 & -\Theta .666490 & 0.317858\end{array}$




$\begin{array}{rrr}\text { Frequencies }(\mathrm{cm}-1): & \\ 26.9603 & 47.8525 & 59.8285 \\ 120.3458 & 148.5992 & 196.6411 \\ 204.5737 & 256.2711 & 307.5401 \\ 374.3888 & 424.7146 & 456.8251 \\ 474.0167 & 496.2067 & 549.7404 \\ 566.2225 & 595.5813 & 606.2351 \\ 635.6157 & 680.1553 & 705.1668 \\ 721.1047 & 733.6894 & 757.6559 \\ 765.6840 & 809.1055 & 825.4985 \\ 839.2809 & 869.9379 & 883.6486 \\ 885.2435 & 901.2787 & 935.5372 \\ 946.1466 & 950.2434 & 975.8628 \\ 1048.2656 & 1053.4726 & 1077.2233 \\ 1112.5700 & 1130.7950 & 1161.9784 \\ 1191.4892 & 1196.3178 & 1222.1073 \\ 1256.7129 & 1285.9931 & 1307.8743 \\ 1347.8706 & 1378.5287 & 1423.5088 \\ 1451.0551 & 1472.1843 & 1503.2173 \\ 1519.6734 & 1562.3105 & 1608.8707 \\ 1616.6315 & 1648.5523 & 1689.3340 \\ 3117.5833 & 3181.8864 & 3190.9158 \\ 320 \odot .7409 & 3211.1658 & 3245.6206 \\ 3268.0281 & 3279.6810 & 3303.5646\end{array}$

Cis -3

Energy: -1028.92561522 hartree

Geometry (angstrom):

\begin{tabular}{|c|c|c|c|}
\hline 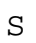 & -0.712409 & $\odot .0 \odot \odot \odot \odot \odot$ & $\odot .251663$ \\
\hline C & -0.639283 & $\odot . \odot \odot \odot \odot \odot \odot$ & 2.002243 \\
\hline C & $\odot .697150$ & $\odot .00 \odot \odot \odot \odot$ & 2.479602 \\
\hline C & 1.642836 & $\odot . \odot \odot \odot \odot \odot \odot$ & 1.412062 \\
\hline c & 1.096677 & $\odot . \odot \odot \odot \odot \odot \odot$ & 0.147884 \\
\hline c & -1.725111 & $\odot . \odot \odot \odot \odot \odot \odot$ & 2.884124 \\
\hline & -1.471848 & $\odot . \odot \odot \odot \odot \odot \odot$ & 4.251802 \\
\hline c & -0.152090 & $\odot . \odot \odot \odot \odot \odot \odot$ & 4.742677 \\
\hline C & $\odot .925864$ & $\odot . \odot \odot \odot \odot \odot \odot$ & 3.870803 \\
\hline $\mathrm{H}$ & 1.944758 & $\odot . \odot \odot \odot \odot \odot \odot$ & 4.249460 \\
\hline $\mathrm{H}$ & $\odot .020340$ & $\odot . \odot \odot \odot \odot \odot \odot$ & 5.815378 \\
\hline 1 & -2.304623 & $\odot . \odot \odot \odot \odot \odot \odot$ & 4.949797 \\
\hline $\mathrm{H}$ & -2.744866 & $\odot . \odot \odot \odot \odot \odot \odot$ & 2.509564 \\
\hline $\mathrm{H}$ & 2.718446 & $\odot . \odot \odot \odot \odot \odot \odot$ & 1.546726 \\
\hline N & 1.910580 & $\odot . ๑ \odot \odot \odot \odot \odot$ & -0.944686 \\
\hline c & 1.709027 & $\odot . \odot \odot \odot \odot \odot \odot$ & -2.217284 \\
\hline $\mathrm{H}$ & 2.625106 & $\odot . ๑ \odot \odot \odot \odot \odot$ & -2.815827 \\
\hline C & $\odot .54820 \odot$ & $\odot . \odot \odot \odot \odot \odot \odot$ & -3.075914 \\
\hline C & $\odot .504531$ & $\odot . \odot \odot \odot \odot \odot \odot$ & -4.454262 \\
\hline C & -0.869385 & $\odot . \odot \odot \odot \odot \odot \odot$ & -4.823965 \\
\hline & -1.573028 & $\odot . \odot \odot \odot \odot \odot \odot$ & -3.653551 \\
\hline b & -0.731088 & $\odot . \odot \odot \odot \odot \odot \odot$ & -2.589383 \\
\hline & 1.362087 & $\odot . \odot \odot \odot \odot \odot \odot$ & -5.112958 \\
\hline & -1.283382 & $\odot . \odot \odot \odot \odot \odot \odot$ & -5.822399 \\
\hline & -2.625916 & $\odot .0000 \odot \odot$ & -3.415804 \\
\hline
\end{tabular}




$\begin{array}{rrr}\text { Frequencies }(\mathrm{cm}-1): & \\ 24.0575 & 41.0976 & 85.4584 \\ 105.5737 & 144.6665 & 188.8854 \\ 212.7683 & 261.1754 & 311.3016 \\ 374.4594 & 428.3312 & 465.6004 \\ 471.7422 & 494.9322 & 544.0536 \\ 559.1030 & 592.1260 & 604.6509 \\ 643.9182 & 652.3311 & 684.5688 \\ 719.4458 & 736.6383 & 759.6476 \\ 761.8370 & 786.9338 & 818.5177 \\ 853.6223 & 879.2539 & 882.3824 \\ 886.7858 & 901.8343 & 928.0854 \\ 940.6534 & 952.0026 & 979.6891 \\ 1046.3852 & 1053.2472 & 1080.1227 \\ 1111.0203 & 1131.9290 & 1161.4873 \\ 1191.1677 & 1194.1955 & 1211.5275 \\ 1256.4702 & 1284.5628 & 1310.2927 \\ 1352.3201 & 1378.4660 & 1428.1555 \\ 1454.9005 & 1468.2258 & 1503.1801 \\ 1525.8830 & 1559.1921 & 1609.3910 \\ 1616.1955 & 1647.4273 & 1692.7913 \\ 3104.0912 & 3184.0794 & 3192.0503 \\ 3202.2527 & 3211.7864 & 3237.3869 \\ 3267.8167 & 3279.4949 & 3306.4139\end{array}$

\section{Compound $3 b$}

Trans-1

Energy: -1351.91537820 hartree

Geometry (angstrom):

\begin{tabular}{|c|c|c|c|}
\hline S & 1. 290286 & -1.267098 & -0.000382 \\
\hline C & 2.918858 & -0.602934 & 0.000036 \\
\hline C & 2.908921 & ๑. 815944 & $-\odot . \odot \odot \odot 19 \odot$ \\
\hline C & 1.579535 & 1.347421 & -0.000385 \\
\hline C & 0.592845 & 0.397009 & -0.000114 \\
\hline C & 4.113615 & -1.325432 & 0.000334 \\
\hline C & 5.315093 & -0.621230 & 0.000411 \\
\hline C & 5.326420 & 0.784757 & ๑. . 000182 \\
\hline C & 4.138220 & 1.502412 & -0.000115 \\
\hline $\mathrm{H}$ & 4.148153 & 2.589255 & $-\odot .0 \odot \odot 266$ \\
\hline $\mathrm{H}$ & 6.275694 & 1.313120 & 0.000233 \\
\hline $\mathrm{H}$ & 6.254255 & -1.167440 & ๑. . 000600 \\
\hline $\mathrm{H}$ & 4.107407 & -2.411545 & ๑. . $00 \odot 427$ \\
\hline $\mathrm{H}$ & 1.342678 & 2.405287 & -0.000465 \\
\hline $\mathrm{N}$ & $-\odot .743289$ & $\odot .687050$ & $\odot .0 \odot \odot \odot 75$ \\
\hline C & -1.649335 & -0.231408 & ๑. .000154 \\
\hline $\mathrm{H}$ & -1.394918 & -1.300491 & -0.000035 \\
\hline C & -3.056077 & $\odot .098967$ & $\odot .0 \odot \odot 13 \odot$ \\
\hline C & -3.656249 & 1.343498 & ๑ . 000191 \\
\hline C & -5.072507 & 1.278949 & ๑ . 000148 \\
\hline C & -5.548520 & -0.009336 & 0.000038 \\
\hline S & -4.266755 & -1.168932 & -0.000017 \\
\hline $\mathrm{H}$ & -3.075529 & 2.258151 & 0.000274 \\
\hline $\mathrm{H}$ & -5.718058 & 2.150479 & ๑ . 000194 \\
\hline $\mathrm{H}$ & -6.578072 & -0.341374 & -0.000014 \\
\hline
\end{tabular}




$\begin{array}{rrr}\text { Frequencies }(\mathrm{cm}-1): & \\ 28.5160 & 43.8339 & 56.7589 \\ 110.1869 & 168.3619 & 179.0614 \\ 206.1330 & 221.6119 & 279.0852 \\ 344.9483 & 355.6174 & 443.3997 \\ 450.0952 & 481.5521 & 491.0312 \\ 518.3720 & 541.7951 & 568.7374 \\ 595.1078 & 603.2754 & 657.0971 \\ 660.7100 & 715.6572 & 717.0858 \\ 733.7739 & 747.6561 & 761.2356 \\ 847.3005 & 849.4447 & 853.6346 \\ 869.0517 & 876.1592 & 885.7509 \\ 926.8214 & 939.4787 & 961.6629 \\ 979.7363 & 1048.6663 & 1061.3706 \\ 1078.4934 & 1111.8342 & 1145.6017 \\ 1160.1038 & 1190.2454 & 1203.2880 \\ 1222.7669 & 1277.0659 & 1285.8012 \\ 1343.4097 & 1375.6541 & 1405.3181 \\ 1425.4509 & 1468.9829 & 1488.3065 \\ 1506.7633 & 1565.5897 & 1594.8131 \\ 1620.7219 & 1645.1678 & 1665.1656 \\ 3048.2054 & 3185.8725 & 3194.2603 \\ 3204.4753 & 3213.6729 & 3226.4510 \\ 3236.5930 & 3250.3610 & 3271.9719\end{array}$

Trans-2

Energy: -1351.91814770 hartree

Geometry (angstrom):

\begin{tabular}{|c|c|c|c|}
\hline i & 0.774984 & -0.017868 & 2.883066 \\
\hline C & $\odot .775414$ & 0.036848 & 4.278167 \\
\hline C & 1.982811 & -0.141438 & 4.948905 \\
\hline C & 3.174481 & -0.371277 & 4.239034 \\
\hline C & 3.173223 & -0.425532 & 2.851931 \\
\hline C & 1.966699 & $-\odot .248694$ & 2.148090 \\
\hline H & -0.143900 & 0.214272 & 4.828732 \\
\hline $\mathrm{H}$ & 2.001584 & -0.101987 & 6.034476 \\
\hline H & 4.104800 & $-\odot .5 \odot 7569$ & 4.783293 \\
\hline H & 4.095023 & -0.603260 & 2.304207 \\
\hline C & 1.736380 & -0.265138 & 0.735078 \\
\hline C & 0.431047 & $-\odot .058046$ & 0.375588 \\
\hline S & -0.616472 & 0.176398 & 1.824676 \\
\hline N & $-\odot .0 \odot 79 \odot 3$ & -0.034458 & $-\odot .919119$ \\
\hline C & -1.244042 & 0.162803 & -1.230641 \\
\hline $\mathrm{H}$ & -2.025337 & 0.324745 & -0.474626 \\
\hline C & -1.696985 & 0.185499 & -2.596483 \\
\hline C & -2.988781 & $\odot .386384$ & -3.045209 \\
\hline C & -3.101771 & 0.349499 & -4.459978 \\
\hline C & -1.896318 & 0.121225 & -5.074119 \\
\hline S & -0.599354 & -0.053352 & -3.940131 \\
\hline $\mathrm{H}$ & 2.507121 & -0.425015 & $-\odot .010286$ \\
\hline $\mathrm{H}$ & -1.697489 & 0.045659 & -6.135241 \\
\hline $\mathrm{H}$ & -4.033145 & 0.485832 & -4.998331 \\
\hline & -3.822973 & $\odot .554198$ & -2.371675 \\
\hline
\end{tabular}




$\begin{array}{rrr}\text { Frequencies }(\mathrm{cm}-1): & \\ 30.8987 & 43.4560 & 55.0468 \\ 107.3518 & 142.8084 & 183.0808 \\ 207.8974 & 249.8800 & 300.3005 \\ 354.0928 & 356.6717 & 443.0764 \\ 458.1495 & 490.0715 & 490.9180 \\ 517.4787 & 545.8866 & 570.6224 \\ 597.8569 & 623.6015 & 644.1609 \\ 698.6588 & 712.6251 & 720.6545 \\ 721.6892 & 733.8116 & 761.2967 \\ 776.0515 & 836.6863 & 849.0199 \\ 872.2156 & 876.5439 & 885.0459 \\ 915.9559 & 939.3846 & 961.3423 \\ 979.7639 & 1049.1430 & 1077.6211 \\ 1079.1471 & 1117.5911 & 1157.8893 \\ 1168.2665 & 1191.1631 & 1215.5992 \\ 1260.6361 & 1268.9374 & 1285.8225 \\ 1341.5617 & 1367.1614 & 1379.5126 \\ 1423.2049 & 1471.8579 & 1490.7433 \\ 1506.2930 & 1566.9361 & 1598.8615 \\ 1620.6229 & 1646.2758 & 1670.4884 \\ 3049.2789 & 3185.7345 & 3194.2652 \\ 3204.1781 & 3213.4407 & 3218.7528 \\ 3233.7262 & 3236.9488 & 3266.8366\end{array}$

Trans - 3

Energy: -1351.91142903 hartree

Geometry (angstrom):

\begin{tabular}{|c|c|c|c|}
\hline & -2.346916 & $-\odot .273744$ & $\odot .233953$ \\
\hline ; & -2.394762 & -0.304913 & 1.989036 \\
\hline C & -1.085833 & -0.305195 & 2.540644 \\
\hline 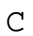 & -0.070265 & -0.260489 & 1.529211 \\
\hline & -0.573155 & $-\odot .220767$ & 0.253821 \\
\hline C & -3.530987 & -0.329111 & 2.801942 \\
\hline C & -3.359999 & $-\odot .364244$ & 4.182514 \\
\hline & -2.071888 & -0.372429 & 4.747653 \\
\hline C & -0.942788 & -0.344734 & 3.941198 \\
\hline H & 0.050894 & -0.348047 & 4.381948 \\
\hline $\mathrm{H}$ & -1.961400 & -0.400016 & 5.828204 \\
\hline H & -4.232496 & -0.387794 & 4.829443 \\
\hline $\mathrm{H}$ & -4.526170 & -0.325237 & 2.366506 \\
\hline $\mathrm{H}$ & 0.992218 & -0.301470 & 1.744505 \\
\hline $\mathrm{N}$ & ๑.058953 & $-\odot .220151$ & -0.971247 \\
\hline C & 1.205498 & 0.355039 & -1.099778 \\
\hline $\mathrm{H}$ & 1.662942 & ๑. 917853 & $-\odot .274464$ \\
\hline C & 1.944478 & $\odot .296595$ & -2.341855 \\
\hline C & 1.615696 & -0.350856 & -3.516782 \\
\hline C & 2.584235 & -0.177260 & -4.537582 \\
\hline C & 3.646657 & 0.596681 & -4.139191 \\
\hline S & 3.477269 & 1.130243 & -2.503975 \\
\hline $\mathrm{H}$ & 0.702423 & $-\odot .925141$ & -3.617222 \\
\hline $\mathrm{H}$ & 2.502636 & $-\odot .608679$ & -5.529253 \\
\hline $\mathbf{H}$ & 4.518233 & 0.881467 & -4.713259 \\
\hline
\end{tabular}




\begin{tabular}{|c|c|c|}
\hline $\begin{array}{r}\text { quencles } \\
31.8396\end{array}$ & 43.5039 & 52.5204 \\
\hline 118.1432 & 154.8801 & 190.0804 \\
\hline 198.1642 & 231.6297 & 278.3042 \\
\hline 326.8040 & 364.1008 & 435.2408 \\
\hline 449.0510 & 486.2935 & 493.8025 \\
\hline 515.8210 & 544.6302 & 573.6553 \\
\hline 588.5621 & 606.4811 & 649.4230 \\
\hline 711.5826 & 718.0505 & 727.6946 \\
\hline 733.9343 & 747.5912 & 757.9287 \\
\hline 821.8274 & 856.3430 & 861.0749 \\
\hline 868.6175 & 872.4635 & 902.7517 \\
\hline 929.9280 & 937.2662 & 977.9918 \\
\hline 992.5697 & 1048.2181 & 1060.1537 \\
\hline 1079.3671 & 1110.6843 & 1138.6590 \\
\hline 1161.3423 & 1191.5363 & 1202.9606 \\
\hline 1223.9928 & 1278.5118 & 1287.5606 \\
\hline 1348.8057 & 1379.9340 & 1406.1196 \\
\hline 1419.5244 & 1468.5912 & 1485.4380 \\
\hline 1505.3761 & 1558.0675 & 1584.3605 \\
\hline 1614.5618 & 1646.8567 & 1659.4485 \\
\hline 3052.7569 & 3183.3702 & 3192.2539 \\
\hline 3201.9545 & 3212.2260 & 3221.9235 \\
\hline 3227.9116 & 3251.2182 & 3272.4269 \\
\hline
\end{tabular}

Trans - 4

Energy: -1351.91400469 hartree

Geometry (angstrom):

\begin{tabular}{|c|c|c|c|}
\hline 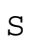 & -2.173035 & -0.118328 & ๑.420918 \\
\hline C & -2.148282 & $-\odot .098707$ & 2.176429 \\
\hline & -0.817787 & $-\odot .095627$ & 2.673874 \\
\hline & ๑.155166 & $-\odot . \odot 89780$ & 1.620551 \\
\hline 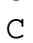 & $-\odot .400172$ & $-\odot .082286$ & $\odot .366547$ \\
\hline & -3.250262 & $-\odot . \odot 8970 \odot$ & 3.035597 \\
\hline & -3.022872 & $-\odot .087559$ & 4.408379 \\
\hline ר & -1.712685 & $-\odot .09168 \odot$ & 4.920413 \\
\hline & -0.617531 & $-\odot . \odot 96856$ & 4.068015 \\
\hline & 0.393388 & $-\odot .097285$ & 4.467696 \\
\hline & -1.558094 & $-\odot .0902 \odot 4$ & 5.995897 \\
\hline & -3.868140 & $-\odot .084936$ & 5.090906 \\
\hline & -4.262492 & $-\odot .08889 \odot$ & 2.641374 \\
\hline 4 & 1.225388 & -0.133709 & 1.792871 \\
\hline & 0.180880 & $-\odot .121572$ & $-\odot .882968$ \\
\hline & 1.329158 & 0.430134 & -1.079227 \\
\hline & 1.841838 & 1.006546 & $-\odot .296596$ \\
\hline & 2.019005 & $\odot .344386$ & -2.340957 \\
\hline C & 3.235753 & $\odot .913301$ & -2.665908 \\
\hline & 3.640046 & 0.644978 & -3.999975 \\
\hline & 2.727565 & -0.125624 & -4.675752 \\
\hline & 1.359171 & -0.540480 & -3.699746 \\
\hline & 3.812672 & 1.504275 & -1.961838 \\
\hline & 4.563059 & 1.006473 & -4.439447 \\
\hline & 2.783939 & -0.473230 & -5.699133 \\
\hline
\end{tabular}




$\begin{array}{rrr}\text { Frequencies }(\mathrm{cm}-1): & \\ 31.5094 & 43.7877 & 52.6986 \\ 111.4116 & 140.7103 & 190.8053 \\ 198.7590 & 258.3952 & 297.1789 \\ 333.2743 & 367.3881 & 437.0253 \\ 450.2045 & 491.3616 & 499.8008 \\ 518.4324 & 547.8394 & 574.5697 \\ 599.4205 & 624.3155 & 666.3863 \\ 684.9625 & 716.4963 & 724.6712 \\ 733.7991 & 743.0999 & 757.6765 \\ 802.6101 & 830.1704 & 840.8071 \\ 869.4247 & 873.2494 & 891.9362 \\ 917.5881 & 937.2630 & 977.9235 \\ 989.5583 & 1049.0327 & 1078.7975 \\ 1079.3410 & 1117.7413 & 1152.5568 \\ 1165.3384 & 1191.4544 & 1217.4470 \\ 1258.5261 & 1273.4863 & 1288.0055 \\ 1348.8883 & 1366.0226 & 1380.2356 \\ 1420.3895 & 1469.7375 & 1489.3043 \\ 1505.2993 & 1555.5826 & 1590.8816 \\ 1614.8871 & 1648.0008 & 1664.2943 \\ 3053.6976 & 3183.4073 & 3192.0520 \\ 3201.9092 & 3212.2077 & 3218.5881 \\ 3221.1777 & 3234.2120 & 3266.7284\end{array}$

\section{Compound $6 \mathrm{~b}$}

\section{Cis-1}

Energy: -1351.90265008 hartree

Geometry (angstrom):

$\begin{array}{rrrr}\text { S } & -2.121389 & -1.107014 & -0.487456 \\ \mathrm{C} & -2.129298 & -1.099230 & 1.271745 \\ \mathrm{C} & -0.808591 & -1.090330 & 1.790507 \\ \mathrm{C} & 0.184875 & -1.055333 & 0.752833 \\ \mathrm{C} & -0.345568 & -1.038096 & -0.508784 \\ \mathrm{C} & -3.246860 & -1.095412 & 2.108886 \\ \mathrm{C} & -3.045031 & -1.102001 & 3.486725 \\ \mathrm{C} & -1.744870 & -1.104004 & 4.020585 \\ \mathrm{C} & -0.633746 & -1.097712 & 3.187102 \\ \mathrm{H} & 0.369669 & -1.095509 & 3.605375 \\ \mathrm{H} & -1.608331 & -1.109502 & 5.098575 \\ \mathrm{H} & -3.902696 & -1.108900 & 4.153525 \\ \mathrm{H} & -4.251647 & -1.096531 & 1.695892 \\ \mathrm{H} & 1.253302 & -1.067242 & 0.938790 \\ \mathrm{~N} & 0.268622 & -1.173741 & -1.738472 \\ \mathrm{C} & 1.162356 & -0.390341 & -2.227392 \\ \mathrm{H} & 1.577934 & -0.725117 & -3.182557 \\ \mathrm{C} & 1.692934 & 0.888521 & -1.768321 \\ \mathrm{C} & 1.367234 & 1.720175 & -0.712179 \\ \mathrm{C} & 2.103093 & 2.932616 & -0.704064 \\ \mathrm{C} & 2.991854 & 3.026449 & -1.746427 \\ \mathrm{~S} & 2.938551 & 1.635590 & -2.762090 \\ \mathrm{H} & 0.623960 & 1.461576 & 0.030201 \\ \mathrm{H} & 1.982068 & 3.704906 & 0.047584 \\ \mathrm{H} & 3.677375 & 3.833990 & -1.966781\end{array}$




Frequencies $(\mathrm{cm}-1):$
21.6286
126.6026
199.2746
363.9585
457.8365
528.8012
588.1637
707.5167
733.2275
819.8928
869.3305
934.1751
976.1276
1077.2977
1155.8679
1217.8987
1343.4609
1466.7402
1505.3505
1616.0452
3110.0065
3200.9452
3229.8239

34.7560

137.5438

239.9336

417.6102

476.9609

552.8336

627.4130

724.1413

745.4637

854.0166

870.9262

935.5213

1046.8427

1105.1051

1165.8280

1280.7865

1377.6565

1468.7664

1565.9091

1648.3329

3182.4411

3211.5032

3265.4304
51.3672

189.1291

296.6595

446.4840

494.3987

571.8276

638.6550

726.9892

757.6641

864.1746

883.5703

963.8864

1056.7470

1124.0236

1190.6266

1286.0507

1400.4394

1491.8402

1579.1421

1691.1779

3191.4665

3225.7246

3271.8244

Cis-2

Energy: -1351.90429661 hartree

Geometry (angstrom) :

$\begin{array}{lrrr}\mathrm{C} & 1.731005 & -0.668020 & 1.672055 \\ \mathrm{C} & 2.186721 & -0.772623 & 2.987609 \\ \mathrm{C} & 2.684572 & 0.368991 & 3.610738 \\ \mathrm{C} & 2.719402 & 1.598883 & 2.931713 \\ \mathrm{C} & 2.257628 & 1.703334 & 1.625702 \\ \mathrm{C} & 1.749462 & 0.565631 & 0.972197 \\ \mathrm{H} & 2.161060 & -1.723707 & 3.511920 \\ \mathrm{H} & 3.051261 & 0.305017 & 4.631412 \\ \mathrm{H} & 3.111267 & 2.478303 & 3.435504 \\ \mathrm{H} & 2.282604 & 2.657702 & 1.105917 \\ \mathrm{C} & 1.205739 & 0.448786 & -0.353419 \\ \mathrm{C} & 0.782352 & -0.816112 & -0.657269 \\ \mathrm{~S} & 1.072996 & -1.957963 & 0.671943 \\ \mathrm{~N} & 0.354158 & -1.341602 & -1.863586 \\ \mathrm{C} & -0.718740 & -0.980486 & -2.469940 \\ \mathrm{H} & -0.875219 & -1.451292 & -3.445417 \\ \mathrm{C} & -1.821130 & -0.101623 & -2.107241 \\ \mathrm{C} & -2.941863 & 0.045191 & -2.906149 \\ \mathrm{C} & -3.947975 & 0.866563 & -2.338241 \\ \mathrm{C} & -3.588650 & 1.342428 & -1.103415 \\ \mathrm{~S} & -2.016152 & 0.806776 & -0.619406 \\ \mathrm{H} & 1.159426 & 1.268442 & -1.061797 \\ \mathrm{H} & -4.158462 & 1.990266 & -0.449523 \\ \mathrm{H} & -4.892128 & 1.093604 & -2.820335 \\ \mathrm{H} & -3.030916 & -0.443367 & -3.871313\end{array}$




$\begin{array}{rrr}\text { Frequencies }(\mathrm{cm}-1): & \\ 25.1357 & 43.3507 & 46.9627 \\ 117.4025 & 135.4933 & 193.7755 \\ 200.3258 & 251.7204 & 308.6928 \\ 367.1293 & 421.4353 & 447.3675 \\ 457.3879 & 487.8640 & 494.6936 \\ 536.2026 & 555.9795 & 576.3316 \\ 598.4592 & 629.6195 & 658.5800 \\ 687.7388 & 714.3519 & 728.2510 \\ 732.6774 & 741.6146 & 757.5403 \\ 787.0773 & 828.9893 & 847.4401 \\ 868.6879 & 870.4645 & 882.4062 \\ 922.6259 & 934.4048 & 968.7782 \\ 975.8326 & 1048.9152 & 1076.2382 \\ 1085.4413 & 1113.4627 & 1124.8669 \\ 1161.6955 & 1190.5283 & 1218.0733 \\ 1238.7202 & 1259.0953 & 1285.8325 \\ 1343.0400 & 1377.5699 & 1381.9497 \\ 1453.7150 & 1473.2054 & 1489.8664 \\ 1504.9719 & 1561.4621 & 1581.8117 \\ 1617.1104 & 1648.8314 & 1696.1141 \\ 3110.7042 & 3183.0418 & 3192.0231 \\ 3201.5062 & 3211.7420 & 3218.4946 \\ 3228.1155 & 3236.0595 & 3265.5510\end{array}$

Cis -4

Energy: -1351.90179235 hartree

Geometry (angstrom) :

$\begin{array}{lrrr}\text { S } & -0.748082 & 0.481883 & 0.461821 \\ \mathrm{C} & -0.745244 & 0.459592 & 2.21930 \odot \\ \mathrm{C} & 0.573506 & 0.437371 & 2.741231 \\ \mathrm{C} & 1.561783 & 0.488188 & 1.707888 \\ \mathrm{C} & 1.055277 & 0.497472 & 0.432319 \\ \mathrm{C} & -1.862661 & 0.459371 & 3.057392 \\ \mathrm{C} & -1.659740 & 0.426937 & 4.434627 \\ \mathrm{C} & -0.360078 & 0.399896 & 4.971049 \\ \mathrm{C} & 0.750183 & 0.406052 & 4.137946 \\ \mathrm{H} & 1.754218 & 0.389592 & 4.553939 \\ \mathrm{H} & -0.225593 & 0.375389 & 6.048860 \\ \mathrm{H} & -2.517717 & 0.421490 & 5.101192 \\ \mathrm{H} & -2.867552 & 0.480638 & 2.645442 \\ \mathrm{H} & 2.629959 & 0.536530 & 1.886713 \\ \mathrm{~N} & 1.854895 & 0.645851 & -0.669507 \\ \mathrm{C} & 1.637458 & 0.362101 & -1.905984 \\ \mathrm{H} & 2.413873 & 0.722462 & -2.587630 \\ \mathrm{C} & 0.576004 & -0.391705 & -2.567371 \\ \mathrm{C} & -0.217368 & -1.435205 & -2.131186 \\ \mathrm{C} & -1.046576 & -1.978503 & -3.147814 \\ \mathrm{C} & -0.881205 & -1.357171 & -4.359229 \\ \mathrm{~S} & 0.301546 & -\Theta .101656 & -4.281061 \\ \mathrm{H} & -0.185329 & -1.814701 & -1.118191 \\ \mathrm{H} & -1.734550 & -2.801338 & -2.987591 \\ \mathrm{H} & -1.395008 & -1.561030 & -5.289355\end{array}$




$\begin{array}{rrr}\text { Frequencies }(\mathrm{cm}-1): & \\ 13.2562 & 30.6689 & 72.6645 \\ 125.9284 & 149.3668 & 189.6236 \\ 200.3289 & 221.8380 & 290.1214 \\ 367.5329 & 410.8800 & 442.1696 \\ 464.0327 & 478.3979 & 492.4854 \\ 519.1437 & 555.8999 & 571.0327 \\ 602.0666 & 609.2859 & 638.3731 \\ 671.1227 & 717.8489 & 719.9437 \\ 733.8458 & 743.5745 & 760.8208 \\ 834.5609 & 848.8194 & 853.1505 \\ 866.3519 & 874.3874 & 886.2741 \\ 919.9842 & 933.6608 & 938.5867 \\ 978.7630 & 1045.4253 & 1056.4249 \\ 1079.4398 & 1102.6635 & 1122.8768 \\ 1158.5006 & 1164.5935 & 1190.4105 \\ 1212.0358 & 1280.2565 & 1285.5176 \\ 1346.0332 & 1376.8293 & 1399.3430 \\ 1467.7579 & 1473.3639 & 1486.9790 \\ 1505.4856 & 1565.8713 & 1580.1495 \\ 1617.9159 & 1645.8365 & 1678.6153 \\ 3104.4832 & 3184.9321 & 3192.9872 \\ 3203.1012 & 3212.6464 & 3228.9277 \\ 3235.7940 & 3259.5067 & 3271.0796\end{array}$

\section{Compound $5 \mathbf{a}$}

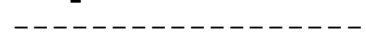

Trans-1

Energy: -1028.93397908 hartree

Geometry (angstrom):

$\begin{array}{rrrr}\text { S } & -1.558665 & 0.370622 & 0.465442 \\ \text { C } & -1.573243 & 0.013094 & 2.187510 \\ \text { C } & -0.313761 & -0.460178 & 2.637316 \\ \text { C } & 0.648380 & -0.526843 & 1.578116 \\ \text { C } & 0.171994 & -0.127177 & 0.358899 \\ \text { C } & -2.654651 & 0.154940 & 3.059422 \\ \text { C } & -2.475538 & -\Theta .180553 & 4.399047 \\ \text { C } & -1.235151 & -\Theta .651333 & 4.864333 \\ \text { C } & -0.160534 & -\Theta .792046 & 3.996762 \\ \text { H } & 0.797915 & -1.156086 & 4.357628 \\ \text { H } & -1.118261 & -0.907099 & 5.913729 \\ \text { H } & -3.306278 & -0.076353 & 5.091446 \\ \text { H } & -3.613818 & 0.518422 & 2.702178 \\ \text { H } & 1.672452 & -0.860645 & 1.701056 \\ \text { N } & 0.924051 & -0.112347 & -0.786462 \\ \text { C } & 0.443333 & 0.270781 & -1.918896 \\ \text { H } & -0.597707 & 0.606876 & -2.027630 \\ \text { C } & 1.247380 & 0.281339 & -3.120532 \\ \text { C } & 2.630274 & -0.102212 & -3.274394 \\ \text { C } & 2.929596 & 0.082323 & -4.582759 \\ \text { O } & 1.838403 & 0.554525 & -5.268465 \\ \text { C } & 0.831491 & 0.668843 & -4.370024\end{array}$




$\begin{array}{rrrr}H & 3.280935 & -0.462217 & -2.491551 \\ H & 3.820131 & -0.061233 & -5.175042 \\ H & -0.109730 & 1.032738 & -4.754737\end{array}$

$\begin{array}{rrr}\text { Frequencies }(\mathrm{cm}-1): & \\ 35.0526 & 46.0930 & 59.4223 \\ 113.1038 & 166.4959 & 183.9519 \\ 207.9013 & 253.1927 & 321.2159 \\ 359.5882 & 366.8491 & 444.7425 \\ 467.8150 & 491.1676 & 537.0661 \\ 540.6515 & 572.6207 & 614.7482 \\ 650.1787 & 655.9731 & 668.8685 \\ 716.7452 & 734.0605 & 737.8164 \\ 761.2982 & 793.2602 & 804.8822 \\ 848.8832 & 870.5692 & 876.5656 \\ 884.3537 & 887.6087 & 939.2551 \\ 967.5663 & 979.5931 & 996.0648 \\ 1047.2491 & 1049.5162 & 1078.2883 \\ 1113.6398 & 1157.8347 & 1168.0064 \\ 1191.0099 & 1205.2126 & 1215.1330 \\ 1267.1767 & 1285.4951 & 1319.7043 \\ 1341.9887 & 1374.5018 & 1407.0096 \\ 1439.5191 & 1481.4409 & 1506.0893 \\ 1557.8530 & 1583.1889 & 1618.1557 \\ 1629.1045 & 1649.0621 & 1687.8561 \\ 3043.9893 & 3185.5408 & 3193.9698 \\ 3204.1078 & 3213.4005 & 3235.7909 \\ 3288.5456 & 3294.1987 & 3310.9562\end{array}$

Trans - 2

Energy: -1028.93238479 hartree

Geometry (angstrom) :

\begin{tabular}{|c|c|c|c|}
\hline & -1.627503 & ๑.๑०८९९९ & ๑. 383430 \\
\hline C & -1.637286 & $\odot . \odot \odot \odot \odot \odot \odot$ & 2.142404 \\
\hline & -0.322741 & $\odot . \odot \odot \odot \odot \odot \odot$ & 2.675825 \\
\hline & 0.677115 & $\odot .00 \odot \odot \odot \odot$ & 1.650114 \\
\hline$c$ & $\odot .176582$ & $\odot . \odot \odot \odot \odot \odot \odot$ & 0.376103 \\
\hline & -2.761848 & $\odot . \odot \odot \odot \odot \odot \odot$ & 2.970198 \\
\hline & -2.570705 & $\odot . \odot \odot \odot \odot \odot \odot$ & 4.349626 \\
\hline ? & -1.275975 & $\odot . \odot \odot \odot \odot \odot \odot$ & 4.897591 \\
\hline & -0.158471 & $\odot .00 \odot \odot \odot \odot$ & 4.073877 \\
\hline & 0.841910 & $\odot . \odot \odot \odot \odot \odot \odot$ & 4.498865 \\
\hline$H$ & -1.150627 & $\odot . \odot \odot \odot \odot \odot \odot$ & 5.976761 \\
\hline & -3.434546 & $\odot .00 \odot \odot \odot \odot$ & 5.008550 \\
\hline H & -3.763091 & $\odot . \odot \odot \odot \odot \odot \odot$ & 2.549139 \\
\hline $\mathrm{H}$ & 1.745101 & $\odot . \odot \odot \odot \odot \odot \odot$ & 1.836229 \\
\hline & ๑.956447 & $\odot .0000 \odot \odot$ & -0.750024 \\
\hline C & $\odot .452550$ & $\odot . \odot \odot \odot \odot \odot \odot$ & -1.935928 \\
\hline H & -0.633142 & $\odot . \odot \odot \odot \odot \odot \odot$ & -2.107299 \\
\hline C & 1.281645 & $\odot . \odot \odot \odot \odot \odot \odot$ & -3.124815 \\
\hline C & $\odot .833660$ & $\odot . \odot \odot \odot \odot \odot \odot$ & -4.496582 \\
\hline C & 1.952326 & $\odot .00 \odot \odot \odot \odot$ & -5.264254 \\
\hline & 3.071769 & $\odot . \odot \odot \odot \odot \odot \odot$ & -4.475185 \\
\hline C & 2.653701 & $\odot . \odot \odot \odot \odot \odot \odot$ & -3.188003 \\
\hline & -0.189958 & $\odot .00 \odot \odot \odot \odot$ & -4.845497 \\
\hline & 2.132106 & $\odot . \odot \odot \odot \odot \odot \odot$ & -6.328077 \\
\hline & 3.419698 & $\odot . \diamond \odot \odot \odot \odot \odot$ & -2.428848 \\
\hline
\end{tabular}

S39 


\begin{tabular}{|c|c|c|}
\hline $\begin{array}{r}\text { quencles } \\
32.3481\end{array}$ & 44.5695 & 59.6335 \\
\hline 112.8214 & 165.3953 & 174.7496 \\
\hline 203.8434 & 253.4785 & 312.4794 \\
\hline 358.6873 & 364.6167 & 444.1840 \\
\hline 466.4522 & 490.9066 & 536.6386 \\
\hline 539.0464 & 572.0319 & 619.5404 \\
\hline 649.9294 & 650.9643 & 667.7297 \\
\hline 716.7719 & 733.4478 & 734.2674 \\
\hline 761.7500 & 808.3976 & 809.8027 \\
\hline 847.9789 & 850.8417 & 876.2071 \\
\hline 884.4429 & 889.3256 & 939.3844 \\
\hline 972.5029 & 980.0742 & 1012.0611 \\
\hline 1049.1376 & 1059.2989 & 1078.3344 \\
\hline 1116.2614 & 1156.0976 & 1164.0508 \\
\hline 1190.5468 & 1207.2340 & 1219.0779 \\
\hline 1261.4906 & 1285.4879 & 1305.4143 \\
\hline 1342.9468 & 1374.5416 & 1393.7889 \\
\hline 1443.8695 & 1483.3985 & 1506.8219 \\
\hline 1548.6234 & 1579.8887 & 1615.7136 \\
\hline 1634.2643 & 1647.8461 & 1683.5796 \\
\hline 3046.8337 & 3185.5006 & 3193.8224 \\
\hline 3204.0153 & 3213.2187 & 3235.9766 \\
\hline 3267.9770 & 3308.1244 & 3316.3025 \\
\hline
\end{tabular}

Trans-3

Energy: -1028.93011247 hartree

Geometry (angstrom):

\begin{tabular}{|c|c|c|c|}
\hline 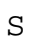 & -2.092083 & -0.127292 & -0.014633 \\
\hline C & -2.195386 & $-\odot .176999$ & 1.738151 \\
\hline & -0.905303 & -0.231534 & 2.329181 \\
\hline & 0.142106 & $-\odot .211509$ & 1.348620 \\
\hline$C$ & $-\odot .319961$ & -0.138685 & $\odot .060591$ \\
\hline & -3.356626 & $-\odot .169396$ & 2.515168 \\
\hline & -3.230185 & $-\odot .227378$ & 3.899826 \\
\hline ר & -1.961527 & $-\odot .289032$ & 4.503790 \\
\hline & -0.807434 & $-\odot .292605$ & 3.732548 \\
\hline & 0.171240 & -0.337424 & 4.203624 \\
\hline & -1.885600 & $-\odot .333620$ & 5.586767 \\
\hline & -4.122701 & -0.227034 & 4.519304 \\
\hline & -4.336977 & -0.123929 & 2.049459 \\
\hline 4 & 1.195765 & $-\odot .292579$ & 1.593965 \\
\hline & $\odot .353533$ & $-\odot .15 \odot 930$ & -1.145263 \\
\hline & 1.504413 & 0.418921 & -1.237088 \\
\hline $\mathrm{H}$ & 1.937172 & $\odot .982450$ & $-\odot .398617$ \\
\hline & 2.287942 & ๑.358132 & -2.452218 \\
\hline C & 1.966900 & $-\odot .309804$ & -3.690395 \\
\hline C & 3.007459 & $-\odot .075017$ & -4.525498 \\
\hline & 3.963893 & $\odot .690783$ & -3.905705 \\
\hline C & 3.512566 & 0.943492 & -2.654987 \\
\hline & 1.070775 & $-\odot .877297$ & -3.892415 \\
\hline & 3.230624 & -0.357583 & -5.542705 \\
\hline & 4.157370 & 1.542425 & -2.028903 \\
\hline
\end{tabular}




$\begin{array}{rrr}\text { Frequencies }(\mathrm{cm}-1): & \\ 39.4323 & 44.6817 & 55.5501 \\ 121.0362 & 157.8787 & 194.1667 \\ 199.4650 & 262.3712 & 307.0211 \\ 347.8901 & 382.8614 & 442.6168 \\ 455.8505 & 493.4917 & 540.0154 \\ 547.7333 & 582.4713 & 615.7227 \\ 652.6101 & 656.0106 & 700.3405 \\ 719.0270 & 734.1220 & 740.5358 \\ 757.5680 & 796.4061 & 815.1397 \\ 841.7766 & 869.7716 & 873.8961 \\ 886.4464 & 893.2684 & 936.8785 \\ 977.5382 & 994.2342 & 999.4126 \\ 1045.6817 & 1049.4972 & 1079.1526 \\ 1113.7194 & 1154.2526 & 1167.8647 \\ 1191.2519 & 1205.0348 & 1220.7958 \\ 1266.2870 & 1285.6760 & 1320.3874 \\ 1347.9633 & 1379.5100 & 1405.3460 \\ 1436.3334 & 1476.4065 & 1505.1175 \\ 1554.3888 & 1568.7486 & 1614.0866 \\ 1626.3322 & 1650.0344 & 1683.6661 \\ 3049.7295 & 3183.2057 & 3191.9894 \\ 3201.7968 & 3212.0989 & 3221.2884 \\ 3289.0463 & 3293.7396 & 3312.1374\end{array}$

Trans - 4

Energy: -1028.92852374 hartree

Geometry (angstrom):

\begin{tabular}{|c|c|c|c|}
\hline & -2.094014 & -0.103089 & -0.004549 \\
\hline C & -2.189583 & -0.153029 & 1.748799 \\
\hline & -0.897410 & -0.222107 & 2.333683 \\
\hline & $\odot .145619$ & $-\odot .213165$ & 1.348297 \\
\hline$C$ & -0.321626 & -0.134236 & 0.062471 \\
\hline & -3.347023 & -0.133409 & 2.531190 \\
\hline & -3.214769 & $-\odot .193936$ & 3. 915227 \\
\hline & -1.944072 & -0.270092 & 4.513167 \\
\hline & -0.793695 & $-\odot .285615$ & 3.736514 \\
\hline & 0.186625 & -0.341615 & 4.202940 \\
\hline & -1.863566 & $-\odot .316560$ & 5.595737 \\
\hline & -4.104331 & -0.184288 & 4.538864 \\
\hline & -4.328975 & -0.076784 & 2.070106 \\
\hline 4 & 1.199431 & $-\odot .306891$ & 1.588299 \\
\hline & ๑.347151 & $-\odot .152043$ & -1.145498 \\
\hline & 1.501088 & 0.410989 & -1.241999 \\
\hline $\mathrm{H}$ & 1.936221 & $\odot .975702$ & -0.405699 \\
\hline & 2.293413 & 0.344322 & -2.455276 \\
\hline C & 3.570426 & $\odot .971561$ & -2.696499 \\
\hline & 3.924862 & 0.640810 & -3.963625 \\
\hline & 2.963683 & $-\odot .149969$ & -4.536070 \\
\hline ; & 1.987020 & -0.321814 & -3.616310 \\
\hline & 4.133251 & 1.585478 & -2.006678 \\
\hline & 4.775848 & 0.864924 & -4.587768 \\
\hline & 1.144694 & -0.928160 & -3.909803 \\
\hline
\end{tabular}




$\begin{array}{rrr}\text { Frequencies }(\mathrm{cm}-1): & \\ 34.3212 & 44.5627 & 55.1649 \\ 122.7159 & 152.9844 & 188.8373 \\ 198.8533 & 258.1634 & 299.5158 \\ 345.9043 & 382.6816 & 441.5738 \\ 454.3025 & 493.3140 & 537.6942 \\ 547.7119 & 581.8047 & 620.2524 \\ 649.7947 & 652.4513 & 701.9669 \\ 719.4260 & 734.0547 & 735.2912 \\ 757.5259 & 814.0919 & 816.1235 \\ 842.9711 & 850.9620 & 869.7850 \\ 887.5235 & 893.9323 & 936.8357 \\ 977.5140 & 1005.1522 & 1012.2104 \\ 1048.9444 & 1058.0765 & 1078.9681 \\ 1116.4451 & 1150.5323 & 1164.9564 \\ 1191.2730 & 1208.9004 & 1221.3472 \\ 1260.2694 & 1285.5385 & 1307.5311 \\ 1347.8923 & 1379.5225 & 1392.3207 \\ 1440.4465 & 1478.8130 & 1505.4542 \\ 1548.2223 & 1565.2072 & 1614.5238 \\ 1625.4540 & 1649.7537 & 1680.5559 \\ 3051.7925 & 3183.1985 & 3191.9839 \\ 3201.7666 & 3212.0820 & 3221.7035 \\ 3268.0940 & 3309.5229 & 3316.3091\end{array}$

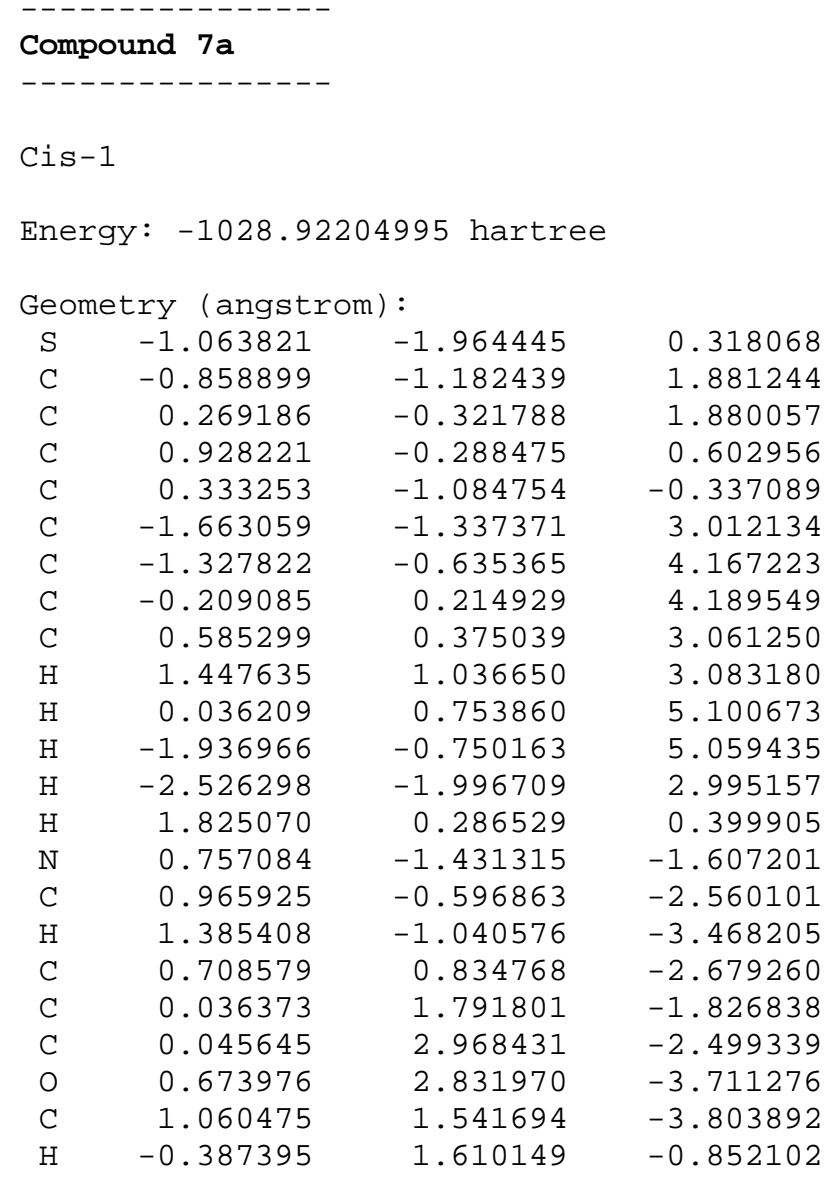




$\begin{array}{rrrr}H & -0.328270 & 3.956503 & -2.279706 \\ \mathrm{H} & 1.569806 & 1.260690 & -4.714182\end{array}$

Frequencies $(\mathrm{cm}-1)$ :

25.6632
130.1733
212.1814
372.8843
478.2358
562.7708
633.8866
719.5365
757.0219
834.7053
881.4489
970.9074
1048.9203
1118.6606
1190.5703
1266.5274
1343.0764
1464.8862
1551.0008
1619.5543
3109.2872
3200.6270
3291.8494

40.7997

57.6373

144.2649

261.9516

427.5195

494.9029

598.1914

656.2494

733.1363

800.9617

868.2368

887.5175

976.0241

1052.2725

1133.2099

1213.1343

1285.7814

1377.2475

1485.0075

1566.9232

1649.1189

3182.1450

3211.2537

3298.8309

199.3286

309.6507

456.7341

538.1769

618.7328

702.4150

743.0493

808.6458

878.8650

934.3325

985.3952

1076.3761

1162.4307

1218.1644

1311.4740

1408.3142

1504.7877

1612.5920

1709.9216

3191.1906

3226.0885

3318.3661

Cis - 2

Energy: -1028.92119411 hartree

Geometry (angstrom):

$\begin{array}{lrrr}\mathrm{S} & -1.820041 & -0.824263 & -0.782318 \\ \mathrm{C} & -1.882090 & -0.927945 & 0.974505 \\ \mathrm{C} & -0.585028 & -1.093705 & 1.524192 \\ \mathrm{C} & 0.438469 & -1.108482 & 0.512980 \\ \mathrm{C} & -0.049720 & -0.957404 & -0.754653 \\ \mathrm{C} & -3.019060 & -0.856632 & 1.781590 \\ \mathrm{C} & -2.862272 & -0.967522 & 3.160987 \\ \mathrm{C} & -1.586996 & -1.140367 & 3.725075 \\ \mathrm{C} & -0.455676 & -1.203174 & 2.921001 \\ \mathrm{H} & 0.528641 & -1.334174 & 3.363456 \\ \mathrm{H} & -1.485097 & -1.225119 & 4.803565 \\ \mathrm{H} & -3.736285 & -0.922086 & 3.804611 \\ \mathrm{H} & -4.004831 & -0.724989 & 1.344494 \\ \mathrm{H} & 1.493272 & -1.251416 & 0.721238 \\ \mathrm{~N} & 0.602727 & -1.069759 & -1.972529 \\ \mathrm{C} & 1.460992 & -0.220302 & -2.405600 \\ \mathrm{H} & 1.920531 & -0.483501 & -3.362712 \\ \mathrm{C} & 1.914842 & 1.065055 & -1.877065 \\ \mathrm{C} & 2.840855 & 1.924806 & -2.579214 \\ \mathrm{C} & 2.982939 & 3.040724 & -1.824078 \\ \mathrm{O} & 2.215540 & 2.946203 & -0.693932 \\ \mathrm{C} & 1.578571 & 1.754117 & -0.734490 \\ \mathrm{H} & 3.318770 & 1.716932 & -3.526838 \\ \mathrm{H} & 3.553630 & 3.950567 & -1.925688 \\ \mathrm{H} & 0.938439 & 1.530788 & 0.102752\end{array}$




$\begin{array}{rrr}\text { Frequencies }(\mathrm{cm}-1): & \\ 14.1152 & 43.7202 & 50.5662 \\ 125.8275 & 140.0155 & 197.7262 \\ 214.7231 & 260.9326 & 310.1228 \\ 371.1036 & 428.6343 & 458.0981 \\ 481.2309 & 494.2588 & 535.3959 \\ 561.6995 & 599.8498 & 620.5200 \\ 632.5707 & 648.0813 & 704.6827 \\ 721.2572 & 732.5797 & 741.4613 \\ 756.5501 & 801.2618 & 822.7007 \\ 837.4947 & 854.7946 & 868.3498 \\ 879.6416 & 890.7644 & 933.9961 \\ 975.9697 & 981.9345 & 1003.6783 \\ 1048.8627 & 1058.4177 & 1075.8306 \\ 1115.4710 & 1123.5341 & 1162.1139 \\ 1189.8431 & 1205.3850 & 1216.2908 \\ 1258.1627 & 1285.0773 & 1292.8479 \\ 1341.9476 & 1376.7600 & 1395.0246 \\ 1462.6757 & 1488.3955 & 1505.0794 \\ 1546.3310 & 1575.7910 & 1613.5321 \\ 1617.5029 & 1649.0897 & 1716.0829 \\ 3117.1511 & 3182.8573 & 3191.7806 \\ 3201.2906 & 3211.9170 & 3223.9688 \\ 3267.9390 & 3310.5920 & 3329.1040\end{array}$

\section{Compound $5 b$}

Trans-1

Energy: -1351.91628172 hartree

Geometry (angstrom):

\begin{tabular}{|c|c|c|c|}
\hline b & -1.756870 & -0.000175 & 0.771701 \\
\hline 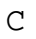 & -1.738231 & $-\odot .00 \odot 230$ & 2.530348 \\
\hline$C$ & -0.415268 & -0.000324 & 3.042776 \\
\hline C & 0.567787 & $-\odot . \odot \odot \odot 258$ & 2.001097 \\
\hline C & 0.046452 & $-\odot .00 \odot \odot 5 \odot$ & $\odot .735366$ \\
\hline C & -2.849446 & $-\odot .0 \odot \odot 249$ & 3.375972 \\
\hline C & -2.636188 & $-\odot .000365$ & 4.752076 \\
\hline C & -1.332833 & $-\odot .0 \odot \odot 462$ & 5.279463 \\
\hline C & -0.228635 & $-\odot .0 \odot \odot 44 \odot$ & 4.438153 \\
\hline $\mathrm{H}$ & 0.778478 & -0.000508 & 4.846895 \\
\hline $\mathrm{H}$ & -1.190391 & $-\odot .0 \odot \odot 556$ & 6.356493 \\
\hline $\mathrm{H}$ & -3.489373 & -0.000395 & 5.424733 \\
\hline $\mathrm{H}$ & -3.857254 & $-\odot . \odot \odot \odot 199$ & 2.970951 \\
\hline H & 1.638707 & $-\odot . \odot \odot \odot 277$ & 2.169610 \\
\hline $\mathrm{N}$ & 0.807979 & $\odot .0 \odot 0163$ & -0.403238 \\
\hline C & ๑. 285839 & $\odot .0 \odot \odot 323$ & -1.581130 \\
\hline H & $-\odot .802226$ & $\odot .000212$ & -1.737115 \\
\hline C & 1.103094 & $\odot .000333$ & -2.780818 \\
\hline C & 2.541858 & $\odot .000325$ & -2.797171 \\
\hline C & 3.054840 & $\odot .00 \odot 374$ & -4.057691 \\
\hline s & 1.799418 & $\odot . \odot \odot \odot 4 \odot \odot$ & -5.267410 \\
\hline C & 0.572415 & $\odot .000346$ & -4.053940 \\
\hline H & 3.135184 & ๑. 000307 & -1.891152 \\
\hline
\end{tabular}




$\begin{array}{rrrr}H & 4.093360 & 0.000396 & -4.360475 \\ H & -0.473215 & 0.000344 & -4.332948\end{array}$

$\begin{array}{rrr}\text { Frequencies }(\mathrm{cm}-1): & \\ 32.9631 & 43.8587 & 55.6915 \\ 100.3421 & 164.4074 & 182.2631 \\ 207.0948 & 235.6897 & 302.9339 \\ 355.3851 & 360.3351 & 443.7961 \\ 458.7200 & 462.1412 & 491.1289 \\ 516.8818 & 538.1767 & 570.9017 \\ 618.7587 & 645.7235 & 652.3942 \\ 664.8023 & 697.2328 & 716.7614 \\ 734.1105 & 761.3973 & 773.0039 \\ 796.3714 & 831.5279 & 849.1987 \\ 874.6347 & 876.6643 & 886.5481 \\ 916.4432 & 939.5797 & 966.1321 \\ 973.2413 & 979.8607 & 1049.1763 \\ 1078.1969 & 1110.4174 & 1156.8626 \\ 1163.4356 & 1190.9482 & 1207.9834 \\ 1218.2793 & 1276.1353 & 1285.5182 \\ 1342.1748 & 1374.2290 & 1398.7208 \\ 1443.1867 & 1474.7212 & 1492.5628 \\ 1507.1656 & 1570.5812 & 1599.3843 \\ 1622.5245 & 1647.4580 & 1677.0304 \\ 3046.0021 & 3185.7636 & 3194.1562 \\ 3204.3455 & 3213.5818 & 3235.8840 \\ 3249.3995 & 3264.9916 & 3274.1973\end{array}$

Trans - 2

Energy: -1351.91462937 hartree

Geometry (angstrom):

\begin{tabular}{|c|c|c|c|}
\hline S & -1.835251 & ๑. . 000000 & ๑. 688075 \\
\hline C & -1.860166 & $\odot .0 \odot \odot \odot \odot \odot$ & 2.446882 \\
\hline C & -0.550232 & $\odot .00000 \odot$ & 2.991705 \\
\hline C & ๑. 458298 & ०. . 000000 & 1.974732 \\
\hline C & -0.031265 & $\odot .000000$ & ๑. 696266 \\
\hline C & -2.991654 & $\odot . ๑ \odot \odot \odot \odot \odot$ & 3.265110 \\
\hline C & -2.812082 & $\odot .00 \odot \odot \odot \odot$ & 4.646113 \\
\hline C & -1.522099 & $\odot . \odot \odot \odot \odot \odot \odot$ & 5.205288 \\
\hline C & -0.397682 & 0.000000 & 4.391186 \\
\hline $\mathrm{H}$ & ๑. 599207 & ๑. . 000000 & 4.824262 \\
\hline $\mathrm{H}$ & -1.406149 & ๑. . 000000 & 6.285490 \\
\hline $\mathrm{H}$ & -3.681490 & $\odot .00000 \odot$ & 5.297682 \\
\hline $\mathrm{H}$ & -3.989342 & ๑. . & 2.835762 \\
\hline $\mathrm{H}$ & 1.524671 & 0.000000 & 2.169902 \\
\hline $\mathrm{N}$ & 0.757809 & ๑. . 000000 & -0.422782 \\
\hline C & ๑. 265971 & $\odot .00 \odot \odot \odot \odot$ & -1.613152 \\
\hline $\mathrm{H}$ & -0.818740 & $\odot .000000$ & -1.792658 \\
\hline C & 1.103144 & $\odot . \odot \odot \odot \odot \odot \odot$ & -2.803068 \\
\hline C & $\odot .575869$ & 0.000000 & -4.140717 \\
\hline C & 1.546657 & ๑. . 000000 & -5.098098 \\
\hline S & 3.134949 & $\odot . ๑ \odot \odot \odot \odot \odot$ & -4.388818 \\
\hline C & 2.483018 & $\odot .000000$ & -2.795417 \\
\hline $\mathrm{H}$ & -0.486116 & ०. . 000000 & -4.363906 \\
\hline $\mathrm{H}$ & 1.431829 & ๑. . 000000 & -6.173467 \\
\hline $\mathrm{H}$ & 3.124983 & 0.000000 & -1.925942 \\
\hline
\end{tabular}




$\begin{array}{rrr}\text { Frequencies }(\mathrm{cm}-1): & \\ 29.6326 & 40.5118 & 55.6183 \\ 107.4308 & 154.3355 & 176.4591 \\ 204.2001 & 245.7865 & 287.2320 \\ 357.3447 & 358.3244 & 443.4269 \\ 465.7009 & 466.0386 & 490.8291 \\ 516.7501 & 537.0970 & 570.7888 \\ 610.2596 & 642.8753 & 652.2562 \\ 670.2435 & 694.6666 & 716.0798 \\ 734.1801 & 749.1845 & 761.6468 \\ 809.0791 & 845.7791 & 848.9687 \\ 876.3968 & 883.5183 & 890.7543 \\ 894.2363 & 939.4669 & 973.9758 \\ 979.9621 & 990.5677 & 1049.2176 \\ 1077.7790 & 1116.6286 & 1155.4247 \\ 1162.4286 & 1191.1379 & 1207.9004 \\ 1215.0027 & 1252.8473 & 1285.6408 \\ 1343.4331 & 1374.8817 & 1389.2138 \\ 1446.6295 & 1474.4127 & 1485.0649 \\ 1506.8481 & 1566.7068 & 1597.2172 \\ 1623.4799 & 1647.4028 & 1679.4515 \\ 3042.9229 & 3185.8195 & 3194.1582 \\ 3204.3751 & 3213.5341 & 3221.4464 \\ 3236.5771 & 3275.3609 & 3286.1875\end{array}$

Trans - 3

Energy: -1351.91234856 hartree

Geometry (angstrom):

\begin{tabular}{|c|c|c|c|}
\hline S & -2.336034 & -0.244117 & ๑. 384942 \\
\hline C & -2.351790 & -0.249675 & 2.141223 \\
\hline C & -1.033229 & -0.238331 & 2.668613 \\
\hline C & -0.036494 & -0.204592 & 1.637349 \\
\hline C & -0.563207 & -0.184726 & ○. 372212 \\
\hline C & -3.473095 & -0.265188 & 2.974793 \\
\hline C & -3.276943 & -0.280109 & 4.352396 \\
\hline C & -1.978828 & -0.276489 & 4.893890 \\
\hline C & -0.864582 & -0.257086 & 4.066545 \\
\hline $\mathrm{H}$ & 0.136936 & -0.251317 & 4.489200 \\
\hline $\mathrm{H}$ & -1.848528 & -0.288313 & 5.972526 \\
\hline $\mathrm{H}$ & -4.137437 & -0.296925 & 5.015413 \\
\hline $\mathrm{H}$ & -4.476025 & -0.270276 & 2.557522 \\
\hline $\mathrm{H}$ & 1.030178 & -0.237876 & 1.832259 \\
\hline $\mathrm{N}$ & ๑. . 050380 & -0.203407 & -0.864660 \\
\hline C & 1.177888 & 0.396342 & -1.027428 \\
\hline $\mathrm{H}$ & 1.636173 & ๑. 990411 & -0.224577 \\
\hline C & 1.906271 & 0.332147 & -2.283024 \\
\hline C & 1.475776 & -0.391775 & -3.449053 \\
\hline C & 2.347059 & -0.280840 & -4.488736 \\
\hline S & 3.722871 & 0.708249 & -4.077654 \\
\hline C & 3.109583 & 0.973918 & -2.487181 \\
\hline $\mathrm{H}$ & 0.552721 & -0.957643 & -3.476673 \\
\hline $\mathrm{H}$ & 2.274331 & -0.717290 & -5.475850 \\
\hline $\mathrm{H}$ & 3.659150 & 1.585396 & -1.783385 \\
\hline
\end{tabular}




$\begin{array}{rrr}\text { Frequencies }(\mathrm{cm}-1): & \\ 35.5387 & 41.2802 & 50.2779 \\ 111.2964 & 153.0756 & 189.0887 \\ 198.6927 & 248.8199 & 297.6050 \\ 337.0410 & 374.1188 & 439.3613 \\ 453.6412 & 462.4711 & 492.7132 \\ 515.0856 & 545.9393 & 579.6028 \\ 616.1725 & 646.0724 & 654.2411 \\ 691.2393 & 698.7389 & 717.8799 \\ 734.1019 & 757.6914 & 798.2717 \\ 803.1523 & 822.1713 & 842.6029 \\ 869.8743 & 876.3885 & 891.8656 \\ 918.2983 & 937.1491 & 963.9585 \\ 977.8230 & 1003.2029 & 1049.0536 \\ 1078.9564 & 1110.5584 & 1149.6067 \\ 1163.6569 & 1191.3224 & 1206.2538 \\ 1224.1234 & 1277.3872 & 1287.3352 \\ 1348.3143 & 1379.5466 & 1393.5120 \\ 1442.7882 & 1471.2922 & 1491.6825 \\ 1505.6035 & 1558.1365 & 1592.1758 \\ 1615.5145 & 1649.2999 & 1672.3151 \\ 3051.8984 & 3183.2382 & 3192.0936 \\ 3201.8426 & 3212.1810 & 3221.9519 \\ 3250.3461 & 3263.8344 & 3274.4653\end{array}$

Trans - 4

Energy: -1351.91073094 hartree

Geometry (angstrom):

\begin{tabular}{|c|c|c|c|}
\hline 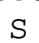 & -2.278963 & -0.173455 & $\odot .460465$ \\
\hline 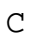 & -2.261011 & $-\odot .157446$ & 2.216773 \\
\hline$C$ & $-\odot .932523$ & $-\odot .153267$ & 2.718730 \\
\hline C & 0.044627 & -0.142582 & 1.668291 \\
\hline C & -0.505964 & -0.132848 & 0.413190 \\
\hline C & -3.366283 & -0.151602 & 3. 071531 \\
\hline C & -3.144088 & -0.152283 & 4.445284 \\
\hline C & -1.835930 & -0.155620 & 4.961907 \\
\hline C & -0.737517 & -0.157263 & 4.113340 \\
\hline $\mathrm{H}$ & 0.271890 & -0.156789 & 4.516838 \\
\hline $\mathrm{H}$ & -1.685133 & -0.156242 & 6.037938 \\
\hline H & -3.991917 & $-\odot .152510$ & 5.124635 \\
\hline $\mathrm{H}$ & -4.377011 & -0.151339 & 2.673449 \\
\hline $\mathrm{H}$ & 1.114473 & -0.185528 & 1.842840 \\
\hline $\mathrm{N}$ & 0.083867 & -0.172972 & -0.834060 \\
\hline C & 1.209268 & 0.420498 & -1.028006 \\
\hline $\mathrm{H}$ & 1.679510 & 1.028098 & -0.241913 \\
\hline C & 1.928042 & $\odot .338180$ & -2.291916 \\
\hline C & 3.164229 & 1.025914 & -2.546563 \\
\hline C & 3.649278 & 0.804888 & -3.801717 \\
\hline S & 2.605137 & -0.252179 & -4.706283 \\
\hline C & 1.513216 & $-\odot .395847$ & -3.383937 \\
\hline $\mathrm{H}$ & 3.658487 & 1.657413 & -1.815423 \\
\hline $\mathrm{H}$ & 4.550887 & 1.195291 & -4.253661 \\
\hline H & $\odot .620892$ & -1.001761 & -3.454622 \\
\hline
\end{tabular}




$\begin{array}{rrr}\text { Frequencies }(\mathrm{cm}-1): & \\ 32.0578 & 41.9560 & 51.8299 \\ 115.0310 & 145.9937 & 184.7752 \\ 198.1782 & 253.0774 & 285.6746 \\ 335.5777 & 374.6968 & 438.0908 \\ 452.2433 & 469.4890 & 493.3344 \\ 523.6210 & 546.2977 & 579.6688 \\ 604.4544 & 643.1083 & 656.2552 \\ 686.5398 & 696.1033 & 717.0566 \\ 734.0178 & 757.4769 & 787.9304 \\ 813.1774 & 825.7401 & 850.0701 \\ 869.7977 & 884.9365 & 894.4993 \\ 896.5971 & 937.0343 & 977.7309 \\ 991.6552 & 1004.9586 & 1049.0932 \\ 1078.9221 & 1115.1827 & 1146.2982 \\ 1162.8337 & 1191.1014 & 1205.8008 \\ 1226.8924 & 1251.0025 & 1286.3155 \\ 1347.9987 & 1379.6102 & 1386.1882 \\ 1444.4750 & 1474.5698 & 1480.3638 \\ 1505.5755 & 1559.9289 & 1585.6275 \\ 1615.3106 & 1649.4663 & 1676.0771 \\ 3048.3587 & 3183.2140 & 3191.9331 \\ 3201.7185 & 3212.0822 & 3221.1664 \\ 3222.0392 & 3275.0783 & 3286.2979\end{array}$

\section{Compound $7 \mathrm{~b}$}

\begin{tabular}{|c|c|c|c|}
\hline \multicolumn{4}{|c|}{ Cis -1} \\
\hline \multicolumn{4}{|c|}{ Energy: -1351.90289053 hartree } \\
\hline \multicolumn{4}{|c|}{ Geometry (angstrom): } \\
\hline $\mathrm{s}$ & -2.144277 & -1.141434 & -0.580233 \\
\hline c & -2.136468 & -1.161893 & 1.179694 \\
\hline $\mathrm{c}$ & -0.811476 & -1.189659 & 1.686364 \\
\hline $\mathrm{c}$ & 0.173462 & -1.162209 & 0.639805 \\
\hline C & -0.368335 & -1.112818 & $-0.61570 \odot$ \\
\hline c & -3.246417 & -1.148313 & 2.026526 \\
\hline c & -3.032940 & -1.180163 & 3.402370 \\
\hline c & -1.728641 & -1.217097 & 3.924350 \\
\hline $\mathrm{c}$ & -0.624845 & -1.221534 & 3.080944 \\
\hline $\mathrm{H}$ & 0.382072 & -1.246624 & 3.489855 \\
\hline $\mathrm{H}$ & -1.582726 & -1.241513 & 5.000846 \\
\hline $\mathrm{H}$ & -3.884813 & -1.178963 & 4.076589 \\
\hline $\mathrm{H}$ & -4.254562 & -1.121762 & 1.622647 \\
\hline $\mathrm{H}$ & 1.243187 & -1.200105 & 0.814177 \\
\hline $\mathrm{N}$ & 0.239643 & -1.234701 & -1.850964 \\
\hline $\mathrm{c}$ & 1.141826 & -0.451007 & -2.318595 \\
\hline $\mathrm{H}$ & 1.569683 & -0.773772 & -3.272412 \\
\hline c & 1.690469 & $\odot .823316$ & -1.840489 \\
\hline C & 1.236354 & 1.666854 & $-\odot .763307$ \\
\hline c & 1.960701 & 2.814745 & -0.650289 \\
\hline $\mathrm{s}$ & 3.222646 & 2.909448 & -1.846066 \\
\hline C & 2.757817 & 1.389705 & -2.509253 \\
\hline
\end{tabular}




$\begin{array}{rrrr}H & 0.409369 & 1.420715 & -0.111698 \\ H & 1.832103 & 3.616582 & 0.064289 \\ H & 3.285244 & 0.980578 & -3.361299\end{array}$

Frequencies (cm-1):

24.8220
124.7425
206.6729
367.3355
460.7185
514.4714
631.6321
697.0520
732.6237
805.9126
868.7260
912.3734
975.2824
1076.0654
1161.8344
1218.9099
1343.0901
1452.9112
1504.8670
1616.5444
3111.4325
3201.3835
3263.3344

31.6997

133.2883

249 . 0047

420.9484

467.4281

553.7410

635.0218

701.7156

756.5883

822.1796

879.8516

934.3311

976.3973

1119.1270

1190.7427

1277.4377

1377.4137

1472.5411

1557.7187

1648.5457

3182.9478

3211.9046

3263.6373
53.8085

198.4319

303.7192

451.6140

494.4282

581.1827

647.6223

717.9003

792.7338

832.4651

887.7510

948.8894

1048.9491

1130.4130

1197.5805

1286.2853

1411.8138

1492.7281

1583.8319

1704.8603

3191.9797

3226.6483

3280.0582

Cis - 2

Energy: -1351.90194340 hartree

Geometry (angstrom):

$\begin{array}{lrrr}\text { S } & -1.955465 & -1.076833 & -0.691775 \\ \mathrm{C} & -2.028049 & -1.177046 & 1.065111 \\ \mathrm{C} & -0.732809 & -1.325823 & 1.623495 \\ \mathrm{C} & 0.297986 & -1.330258 & 0.619618 \\ \mathrm{C} & -0.183044 & -1.186994 & -0.651871 \\ \mathrm{C} & -3.171467 & -1.118934 & 1.863887 \\ \mathrm{C} & -3.023035 & -1.226488 & 3.244596 \\ \mathrm{C} & -1.749588 & -1.382524 & 3.817421 \\ \mathrm{C} & -0.611864 & -1.431586 & 3.021356 \\ \mathrm{H} & 0.371031 & -1.549093 & 3.470601 \\ \mathrm{H} & -1.653948 & -1.464672 & 4.896709 \\ \mathrm{H} & -3.901933 & -1.191385 & 3.882169 \\ \mathrm{H} & -4.155721 & -1.000354 & 1.419658 \\ \mathrm{H} & 1.353366 & -1.456390 & 0.835324 \\ \mathrm{~N} & 0.476959 & -1.303395 & -1.864397 \\ \mathrm{C} & 1.314198 & -0.452676 & -2.332128 \\ \mathrm{H} & 1.773591 & -0.747031 & -3.280736 \\ \mathrm{C} & 1.754067 & 0.868588 & -1.865259 \\ \mathrm{C} & 2.705387 & 1.622114 & -2.640542 \\ \mathrm{C} & 2.982223 & 2.848344 & -2.115476 \\ \mathrm{~S} & 2.085348 & 3.122708 & -0.653245 \\ \mathrm{C} & 1.337694 & 1.573834 & -0.752333 \\ \mathrm{H} & 3.151865 & 1.247876 & -3.556080 \\ \mathrm{H} & 3.652954 & 3.607255 & -2.494456 \\ \mathrm{H} & 0.641768 & 1.260249 & 0.010668\end{array}$




$\begin{array}{rrr}\text { Frequencies }(\mathrm{cm}-1): & \\ 13.4274 & 35.2313 & 45.6378 \\ 122.2987 & 126.9586 & 195.9225 \\ 205.2094 & 249.0933 & 307.5120 \\ 364.9712 & 423.5523 & 452.0775 \\ 457.3807 & 479.2891 & 494.1349 \\ 520.5133 & 551.0899 & 576.7043 \\ 628.2632 & 633.3303 & 652.8279 \\ 697.2750 & 703.2026 & 715.9422 \\ 732.1859 & 753.6651 & 768.6233 \\ 810.3618 & 829.3017 & 847.0970 \\ 868.2848 & 879.3202 & 895.6846 \\ 897.8680 & 933.5490 & 975.7786 \\ 975.9698 & 982.9123 & 1049.0377 \\ 1075.3790 & 1114.6691 & 1122.9875 \\ 1161.1778 & 1190.5117 & 1193.2644 \\ 1217.7047 & 1246.9260 & 1286.0036 \\ 1342.3298 & 1377.1502 & 1394.2226 \\ 1456.8714 & 1479.7600 & 1492.6556 \\ 1504.9287 & 1572.5486 & 1576.8046 \\ 1617.1351 & 1648.7742 & 1716.4078 \\ 3110.6223 & 3183.0456 & 3192.1327 \\ 3201.5001 & 3212.1273 & 3221.6390 \\ 3225.8313 & 3276.4999 & 3302.5098\end{array}$

\section{Compound $3 \mathrm{c}$}

Trans - 1

Energy: -1233.43112674 hartree

Geometry (angstrom):

\begin{tabular}{|c|c|c|c|}
\hline & -1.860218 & $-0.0 \odot \odot 7 \odot 2$ & 1.407014 \\
\hline 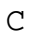 & $-1.93590 \odot$ & $-\odot .001201$ & 3.163157 \\
\hline C & -0.642572 & -0.001057 & 3.748929 \\
\hline & 0.394054 & $-\odot .0 \odot \odot 568$ & 2.764902 \\
\hline & -0.061841 & 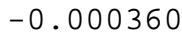 & 1.470576 \\
\hline C & -3.091636 & $-\odot .00167 \odot$ & 3.947035 \\
\hline & -2.951768 & $-\odot . \odot \odot 2 \odot \odot \odot$ & 5.332129 \\
\hline & -1.678946 & $-\odot .001863$ & 5.931660 \\
\hline C & -0.530930 & $-\odot .001397$ & 5.153341 \\
\hline H & 0.452487 & -0.001293 & 5.615747 \\
\hline $\mathrm{H}$ & -1.597179 & $-\odot .0 \odot 2125$ & 7.014733 \\
\hline $\mathrm{H}$ & -3.840117 & -0.002363 & 5.957391 \\
\hline $\mathrm{H}$ & -4.076155 & $-\odot .001770$ & 3.488857 \\
\hline $\mathrm{H}$ & 1.454636 & $-\odot .000392$ & 2.989389 \\
\hline $\mathrm{N}$ & 0.763275 & $\odot .0 \odot \odot \odot 78$ & ๑. 383194 \\
\hline C & ๑.315741 & $\odot .0 \odot \odot 292$ & -0.826727 \\
\hline $\mathrm{H}$ & -0.749637 & $\odot .00 \odot 150$ & -1.086003 \\
\hline C & 1.233073 & $\odot .00 \odot 769$ & -1.935845 \\
\hline C & 2.612202 & $\odot .001092$ & -2.033758 \\
\hline C & 2.919952 & $\odot .001456$ & -3.413510 \\
\hline
\end{tabular}




$\begin{array}{llll}\mathrm{C} & 1.707645 & 0.001335 & -4.055860 \\ \mathrm{O} & 0.676188 & 0.001012 & -3.185163 \\ \mathrm{H} & 3.296620 & 0.001028 & -1.198398 \\ \mathrm{H} & 3.887720 & 0.001737 & -3.891241 \\ \mathrm{~N} & 1.398371 & 0.001532 & -5.448575 \\ \mathrm{O} & 0.213086 & 0.001504 & -5.779070 \\ 0 & 2.369370 & 0.001986 & -6.213419\end{array}$

Frequencies $(\mathrm{cm}-1)$ :

23.9542
75.3046
173.9308
221.5591
356.4693
445.4971
532.9648
580.0758
680.1183
733.4669
810.9757
854.8349
915.3031
985.1186
1047.5212
1159.7459
1217.1068
1286.4511
1370.7730
1444.3408
1517.2013
1624.1642
1667.0196
3197.4538
3238.9262

40.1498
100.0554
184.0861
260.9127
366.3292
474.7674
534.9401
596.3045
680.8257
744.4053
820.5631
880.8480
945.0844
990.6101
1049.3398
1170.8567
1218.0402
1296.8054
1390.3815
1472.2148
1576.8124
1635.7221
3086.2839
3207.9770
3292.6939

42.1729

140.5343

197.9444

319.6534

432.0250

490.9194

569.3432

655.3498

716.7678

763.6164

830.9881

885.2102

955.4210

1004.5020

1078.1091

1193.3928

1246.9499

1344.1467

1404.7393

1505.6053

1611.2357

1645.4427

3189.6499

3216.5092

3308.1828

Trans-2

Energy: -1233.42867302 hartree

Geometry (angstrom):

$\begin{array}{lrrr}\text { S } & -2.385628 & -0.000822 & 1.160348 \\ \mathrm{C} & -2.302309 & -0.001244 & 2.916419 \\ \mathrm{C} & -0.961167 & -0.001047 & 3.382516 \\ \mathrm{C} & -0.016822 & -0.000558 & 2.309549 \\ \mathrm{C} & -0.586087 & -0.000389 & 1.061218 \\ \mathrm{C} & -3.381986 & -0.001713 & 3.802021 \\ \mathrm{C} & -3.116919 & -0.001990 & 5.168799 \\ \mathrm{C} & -1.795008 & -0.001803 & 5.650361 \\ \mathrm{C} & -0.722457 & -0.001336 & 4.770898 \\ \mathrm{H} & 0.299004 & -0.001189 & 5.141578 \\ \mathrm{H} & -1.615160 & -0.002025 & 6.721524 \\ \mathrm{H} & -3.944864 & -0.002355 & 5.872146 \\ \mathrm{H} & -4.404148 & -0.001857 & 3.435310 \\ \mathrm{H} & 1.059803 & -0.000337 & 2.436063 \\ \mathrm{~N} & 0.139486 & 0.000065 & -0.093361 \\ \mathrm{C} & -0.411044 & 0.00 \odot 224 & -1.258489 \\ \mathrm{H} & -1.497715 & 0.0000 \odot 9 & -1.413253 \\ \mathrm{C} & 0.364206 & 0.000709 & -2.470670\end{array}$




$\begin{array}{rrrr}\mathrm{C} & -0.035750 & 0.000946 & -3.795348 \\ \mathrm{C} & 1.143262 & 0.001401 & -4.578240 \\ \mathrm{C} & 2.170336 & 0.001415 & -3.671423 \\ \mathrm{O} & 1.726628 & 0.001021 & -2.397258 \\ \mathrm{H} & -1.057411 & 0.000794 & -4.149125 \\ \mathrm{H} & 1.245379 & 0.001678 & -5.652429 \\ \mathrm{~N} & 3.583699 & 0.001781 & -3.882172 \\ \mathrm{O} & 4.317487 & 0.001648 & -2.895896 \\ 0 & 3.947551 & 0.002059 & -5.063635\end{array}$

$\begin{array}{rrr}\text { Frequencies }(\mathrm{cm}-1): & & \\ 27.0568 & 37.1768 & 41.4590 \\ 68.0581 & 116.2368 & 119.4614 \\ 176.6157 & 183.0864 & 209.4760 \\ 215.4103 & 268.5005 & 339.9178 \\ 366.6224 & 367.4162 & 431.3644 \\ 444.6509 & 467.5516 & 490.9328 \\ 535.2491 & 539.2080 & 557.3524 \\ 569.5178 & 596.8452 & 655.8409 \\ 671.0525 & 689.5076 & 716.4904 \\ 733.4398 & 744.5385 & 763.9713 \\ 812.8256 & 817.0916 & 830.9686 \\ 856.6868 & 882.6889 & 884.9508 \\ 904.1125 & 943.4598 & 946.3049 \\ 985.4797 & 987.8403 & 1004.3047 \\ 1048.3775 & 1051.5943 & 1077.3566 \\ 1159.7596 & 1167.3405 & 1192.8254 \\ 1207.7796 & 1223.2652 & 1275.3264 \\ 1285.7706 & 1308.4056 & 1343.6598 \\ 1369.8344 & 1387.1776 & 1399.0672 \\ 1442.7842 & 1471.3471 & 1503.8414 \\ 1522.2172 & 1578.5060 & 1610.3221 \\ 1616.4816 & 1636.9624 & 1647.8132 \\ 1673.3270 & 3066.7395 & 3189.9103 \\ 3197.6091 & 3207.7426 & 3216.3995 \\ 3241.2011 & 3275.6574 & 3309.1059\end{array}$

Trans - 3

Energy: -1233.41860269 hartree

Geometry (angstrom):

$\begin{array}{rrrr}\mathrm{S} & -2.240981 & 0.093699 & 0.832680 \\ \mathrm{C} & -2.200133 & 0.091039 & 2.584644 \\ \mathrm{C} & -0.866315 & 0.032934 & 3.073671 \\ \mathrm{C} & 0.096893 & 0.020739 & 2.016217 \\ \mathrm{C} & -0.469331 & 0.068468 & 0.764620 \\ \mathrm{C} & -3.294027 & 0.132784 & 3.454667 \\ \mathrm{C} & -3.054158 & 0.104363 & 4.824023 \\ \mathrm{C} & -1.740447 & 0.038581 & 5.326883 \\ \mathrm{C} & -0.653817 & 0.001452 & 4.466956 \\ \mathrm{H} & 0.359538 & -0.046477 & 4.857054 \\ \mathrm{H} & -1.579352 & 0.017377 & 6.401012 \\ \mathrm{H} & -3.892819 & 0.130499 & 5.514052 \\ \mathrm{H} & -4.308634 & 0.180348 & 3.070032 \\ \mathrm{H} & 1.165712 & -0.063132 & 2.182059 \\ \mathrm{~N} & 0.090782 & 0.029970 & -0.489786\end{array}$




$\begin{array}{lrrr}\mathrm{C} & 1.292506 & 0.448372 & -0.673043 \\ \mathrm{H} & 1.887574 & 0.921200 & 0.118682 \\ \mathrm{C} & 1.980166 & 0.394191 & -1.943019 \\ \mathrm{C} & 1.816766 & -0.131943 & -3.216238 \\ \mathrm{C} & 2.913146 & 0.295732 & -4.018131 \\ \mathrm{C} & 3.692729 & 1.042529 & -3.193302 \\ \mathrm{O} & 3.146069 & 1.111661 & -1.949072 \\ \mathrm{~N} & 0.779212 & -1.002161 & -3.716183 \\ \mathrm{H} & 3.071975 & 0.053740 & -5.057203 \\ \mathrm{H} & 4.624202 & 1.570827 & -3.325541 \\ \mathrm{O} & 0.725248 & -1.126252 & -4.944411 \\ \mathrm{O} & 0.047891 & -1.572118 & -2.912633\end{array}$

Frequencies $(\mathrm{cm}-1)$ :

$\begin{array}{rrr}15.9448 & 25.5342 & 39.4925 \\ 61.0771 & 104.7635 & 129.3303 \\ 178.2121 & 197.8979 & 218.5993 \\ 228.0807 & 252.9122 & 289.4287 \\ 340.2195 & 375.4019 & 426.4480 \\ 441.1123 & 453.5245 & 494.1150 \\ 530.9542 & 543.0950 & 558.5792 \\ 579.2616 & 610.0996 & 656.0347 \\ 685.4687 & 711.3907 & 723.2409 \\ 733.5095 & 758.8937 & 759.2745 \\ 781.9817 & 815.8231 & 832.3154 \\ 856.9704 & 871.9208 & 878.7789 \\ 891.6144 & 910.0831 & 941.8683 \\ 975.8996 & 982.1103 & 1047.8475 \\ 1068.8376 & 1079.4053 & 1121.7337 \\ 1155.9634 & 1163.9822 & 1193.1644 \\ 1200.0732 & 1220.0981 & 1243.5296 \\ 1265.7551 & 1286.2506 & 1353.1173 \\ 1380.6774 & 1397.2308 & 1411.1366 \\ 1451.4641 & 1468.6433 & 1505.5882 \\ 1530.1274 & 1553.7660 & 1607.5884 \\ 1614.1720 & 1642.6406 & 1648.7303 \\ 1667.5559 & 3068.6974 & 3186.2018 \\ 3194.6881 & 3204.6309 & 3214.3281 \\ 3222.4785 & 3300.5696 & 3319.1792\end{array}$

Trans -4

Energy: -1233.42349595 hartree

\begin{tabular}{crrr}
\multicolumn{4}{c}{ Geometry (angstrom) : } \\
S & -2.357597 & -0.086643 & 1.079299 \\
C & -2.382431 & -0.038253 & 2.830989 \\
C & -1.068847 & 0.052099 & 3.367197 \\
C & -0.070166 & 0.105587 & 2.344120 \\
C & -0.590805 & 0.060854 & 1.073104 \\
C & -3.507011 & -0.083275 & 3.660700 \\
C & -3.317136 & -0.049426 & 5.037730 \\
C & -2.023252 & 0.031955 & 5.587480 \\
C & -0.906811 & 0.081163 & 4.767251 \\
H & 0.090787 & 0.146516 & 5.193735 \\
H & -1.901199 & 0.056723 & 6.666633 \\
H & -4.179537 & -0.088859 & 5.697130
\end{tabular}




$\begin{array}{rrrr}\mathrm{H} & -4.506095 & -0.148781 & 3.239807 \\ \mathrm{H} & 0.994705 & 0.133817 & 2.550075 \\ \mathrm{~N} & 0.016918 & 0.042169 & -0.160791 \\ \mathrm{C} & 1.183484 & 0.561393 & -0.323057 \\ \mathrm{H} & 1.721035 & 1.093821 & 0.472057 \\ \mathrm{C} & 1.883223 & 0.498656 & -1.580643 \\ \mathrm{C} & 3.113938 & 1.009970 & -1.950697 \\ \mathrm{C} & 3.311554 & 0.658302 & -3.307620 \\ \mathrm{C} & 2.186611 & -0.040437 & -3.658256 \\ \mathrm{O} & 1.313778 & -0.151036 & -2.636500 \\ \mathrm{H} & 3.784740 & 1.570898 & -1.314730 \\ \mathrm{H} & 4.149266 & 0.877178 & -3.951544 \\ \mathrm{~N} & 1.829078 & -0.642113 & -4.905778 \\ \mathrm{O} & 0.748852 & -1.222696 & -4.980698 \\ \mathrm{O} & 2.658841 & -0.519636 & -5.813519\end{array}$

Frequencies $(\mathrm{cm}-1)$ :

$\begin{array}{rrr}21.4243 & 37.1525 & 42.8073 \\ 71.5147 & 105.0924 & 129.9534 \\ 175.4820 & 195.3811 & 200.6687 \\ 215.0185 & 273.9582 & 316.0501 \\ 353.5217 & 380.4874 & 427.7319 \\ 443.2488 & 460.3222 & 493.9277 \\ 533.7003 & 543.8749 & 555.5373 \\ 576.4329 & 598.0166 & 655.4926 \\ 688.9124 & 712.3924 & 726.0002 \\ 733.4643 & 745.3953 & 759.6672 \\ 818.2505 & 819.5533 & 827.8032 \\ 849.6742 & 872.2769 & 893.3014 \\ 905.4646 & 942.6034 & 973.5946 \\ 983.2686 & 987.6771 & 1003.7921 \\ 1047.8885 & 1052.2884 & 1079.4344 \\ 1155.1829 & 1164.7147 & 1193.1082 \\ 1207.6436 & 1222.9287 & 1276.4288 \\ 1285.5552 & 1312.0223 & 1352.8578 \\ 1376.3288 & 1383.5925 & 1397.8210 \\ 1442.7337 & 1463.4104 & 1504.4431 \\ 1522.7284 & 1559.5401 & 1608.4545 \\ 1613.3537 & 1639.1763 & 1648.9138 \\ 1666.1462 & 3069.1302 & 3186.8186 \\ 3195.1861 & 3205.4054 & 3214.8957 \\ 3220.7799 & 3274.7511 & 3308.7309\end{array}$

Cis - 1

Energy: -1233.41745582 hartree

\begin{tabular}{ccrr}
\multicolumn{4}{c}{ Geometry (angstrom): } \\
S & -2.591093 & -1.735490 & -0.332723 \\
C & -2.634612 & -1.734643 & 1.423865 \\
C & -1.334445 & -1.580962 & 1.972418 \\
C & -0.332372 & -1.421494 & 0.957729 \\
C & -0.837779 & -1.456480 & -0.316409 \\
C & -3.762642 & -1.858991 & 2.238593 \\
C & -3.586936 & -1.853611 & 3.619169 \\
C & -2.305138 & -1.716611 & 4.181792 \\
C & -1.185671 & -1.580883 & 3.372812
\end{tabular}




$\begin{array}{rrrr}\mathrm{H} & -0.197122 & -1.470675 & 3.811077 \\ \mathrm{H} & -2.191917 & -1.715736 & 5.262199 \\ \mathrm{H} & -4.451362 & -1.960683 & 4.268227 \\ \mathrm{H} & -4.752851 & -1.968551 & 1.805989 \\ \mathrm{H} & 0.727739 & -1.329254 & 1.167879 \\ \mathrm{~N} & -0.195017 & -1.506537 & -1.535347 \\ \mathrm{C} & 0.718005 & -0.696608 & -1.934962 \\ \mathrm{H} & 1.183164 & -0.930288 & -2.894725 \\ \mathrm{C} & 1.213001 & 0.544940 & -1.359145 \\ \mathrm{C} & 0.914713 & 1.365024 & -0.285644 \\ \mathrm{C} & 1.736508 & 2.511861 & -0.400463 \\ \mathrm{C} & 2.482752 & 2.307435 & -1.530608 \\ \mathrm{O} & 2.186739 & 1.138965 & -2.126015 \\ \mathrm{H} & 0.191692 & 1.155777 & 0.487305 \\ \mathrm{H} & 1.789826 & 3.371417 & 0.249900 \\ \mathrm{~N} & 3.491025 & 3.123940 & -2.135523 \\ \mathrm{O} & 4.023523 & 2.715157 & -3.165002 \\ \mathrm{O} & 3.736483 & 4.185895 & -1.555617\end{array}$

$\begin{array}{rrr}\text { Frequencies }(\mathrm{cm}-1): & \\ 21.7566 & 30.4685 & 47.4722 \\ 77.3836 & 109.9915 & 122.9747 \\ 143.2718 & 199.6302 & 201.7608 \\ 254.6222 & 277.0740 & 299.8386 \\ 366.0583 & 411.6635 & 438.7180 \\ 457.8479 & 480.7418 & 494.8385 \\ 524.9588 & 553.3669 & 578.5951 \\ 599.6224 & 619.4764 & 636.7458 \\ 678.9739 & 705.5411 & 721.0783 \\ 733.1458 & 747.0314 & 759.3198 \\ 810.0444 & 821.4155 & 832.3294 \\ 850.5298 & 872.0138 & 882.7030 \\ 924.3069 & 939.9815 & 955.9885 \\ 981.7107 & 985.8929 & 998.0033 \\ 1048.2300 & 1057.4773 & 1076.8420 \\ 1129.9727 & 1162.8741 & 1192.4832 \\ 1196.7448 & 1221.2413 & 1224.1537 \\ 1286.5712 & 1299.5430 & 1345.8378 \\ 1376.6879 & 1395.0245 & 1410.0192 \\ 1457.3111 & 1481.7198 & 1504.8703 \\ 1507.8240 & 1560.0989 & 1611.1283 \\ 1614.4317 & 1641.8176 & 1648.1402 \\ 1688.0966 & 3147.7593 & 3186.8333 \\ 3195.2714 & 3205.2895 & 3215.2208 \\ 3224.9626 & 3300.9018 & 3311.1713\end{array}$

Cis-2

Energy: -1233.41842595 hartree

\begin{tabular}{cccc}
\multicolumn{4}{c}{ Geometry (angstrom) : } \\
C & -0.403355 & -2.067444 & 2.419824 \\
C & -0.150500 & -2.653339 & 3.665705 \\
C & 0.868199 & -2.127356 & 4.450723 \\
C & 1.634430 & -1.028668 & 4.009711 \\
C & 1.388508 & -0.445518 & 2.778406 \\
C & 0.359574 & -0.958611 & 1.958858
\end{tabular}




$\begin{array}{rrrr}\mathrm{H} & -0.736708 & -3.499648 & 4.011509 \\ \mathrm{H} & 1.077108 & -2.570734 & 5.420433 \\ \mathrm{H} & 2.424562 & -0.638689 & 4.644927 \\ \mathrm{H} & 1.976192 & 0.401165 & 2.434170 \\ \mathrm{C} & -0.046739 & -0.503192 & 0.669635 \\ \mathrm{C} & -1.093803 & -1.228796 & 0.134200 \\ \mathrm{~S} & -1.611898 & -2.529351 & 1.250727 \\ \mathrm{~N} & -1.829191 & -1.212106 & -1.013258 \\ \mathrm{C} & -1.810001 & -0.472697 & -2.069940 \\ \mathrm{H} & -2.556000 & -0.733761 & -2.825731 \\ \mathrm{C} & -1.013757 & 0.651059 & -2.504889 \\ \mathrm{C} & -1.100872 & 1.366321 & -3.690280 \\ \mathrm{C} & -0.106651 & 2.369562 & -3.645470 \\ \mathrm{C} & 0.518712 & 2.198630 & -2.437520 \\ \mathrm{O} & -0.011958 & 1.171903 & -1.738964 \\ \mathrm{H} & 0.429458 & 0.330143 & 0.174887 \\ \mathrm{~N} & 1.595723 & 2.906290 & -1.831033 \\ \mathrm{H} & 0.133556 & 3.120417 & -4.382585 \\ \mathrm{H} & -1.804624 & 1.177644 & -4.488915 \\ \mathrm{O} & 1.973047 & 2.540104 & -0.715057 \\ \mathrm{O} & 2.066240 & 3.841004 & -2.486489\end{array}$

Frequencies (cm-1):

$\begin{array}{rrr}18.7764 & 36.0538 & 54.9561 \\ 78.6935 & 115.6015 & 115.8900 \\ 149.7346 & 199.6179 & 209.4729 \\ 245.2460 & 288.3859 & 305.8110 \\ 370.8489 & 425.9928 & 428.1616 \\ 461.4781 & 464.0894 & 497.5967 \\ 540.9630 & 549.6315 & 569.7829 \\ 593.1331 & 594.0327 & 639.2514 \\ 697.2384 & 703.8325 & 723.8144 \\ 736.0160 & 741.0159 & 765.5433 \\ 801.9534 & 817.1396 & 823.4982 \\ 864.3014 & 889.2552 & 898.8960 \\ 907.6364 & 920.9195 & 951.4176 \\ 987.9166 & 989.8887 & 996.6408 \\ 1045.1461 & 1053.3233 & 1075.7801 \\ 1123.0411 & 1163.5698 & 1196.4690 \\ 1205.3158 & 1236.3055 & 1272.6387 \\ 1285.9093 & 1300.8976 & 1356.1991 \\ 1379.2809 & 1393.2339 & 1405.3003 \\ 1445.9597 & 1465.1502 & 1500.1063 \\ 1515.6233 & 1552.2733 & 1600.2816 \\ 1607.7664 & 1627.2012 & 1641.1964 \\ 1661.0903 & 3114.4308 & 3188.8436 \\ 3197.7173 & 3207.3284 & 3215.1770 \\ 3278.0876 & 3294.2346 & 3306.8411\end{array}$

Cis-3

Energy: -1233.41852799 hartree

Geometry (angstrom) :

$\begin{array}{llll}\text { C } & 1.891425 & -0.000557 & 1.679369 \\ \text { C } & 1.891445 & -0.001781 & 3.078931 \\ \text { C } & 3.113467 & -0.000871 & 3.741343\end{array}$




$\begin{array}{rrrr}\mathrm{C} & 4.328957 & 0.001152 & 3.027229 \\ \mathrm{C} & 4.333120 & 0.002278 & 1.642464 \\ \mathrm{C} & 3.106905 & 0.001392 & 0.943780 \\ \mathrm{H} & 0.958291 & -0.003402 & 3.634439 \\ \mathrm{H} & 3.131039 & -0.001720 & 4.827690 \\ \mathrm{H} & 5.269028 & 0.001803 & 3.571490 \\ \mathrm{H} & 5.268736 & 0.003708 & 1.089510 \\ \mathrm{C} & 2.874511 & 0.001845 & -0.459579 \\ \mathrm{C} & 1.542758 & 0.00 \odot 652 & -0.825315 \\ \mathrm{~S} & 0.484474 & -0.001292 & 0.638557 \\ \mathrm{~N} & 1.212270 & 0.001279 & -2.143871 \\ \mathrm{C} & 0.103732 & 0.00 \odot 961 & -2.801477 \\ \mathrm{H} & 0.220053 & 0.001903 & -3.888797 \\ \mathrm{C} & -1.296367 & 0.00 \odot 385 & -2.442456 \\ \mathrm{C} & -2.396260 & 0.00 \odot 484 & -3.285721 \\ \mathrm{C} & -3.547117 & -0.00 \odot 189 & -2.465300 \\ \mathrm{C} & -3.070926 & -0.00 \odot 652 & -1.180450 \\ \mathrm{O} & -1.719669 & -0.00 \odot 286 & -1.145857 \\ \mathrm{H} & 3.654385 & 0.003227 & -1.212553 \\ \mathrm{~N} & -3.769244 & -0.001337 & 0.063805 \\ \mathrm{H} & -4.586922 & -0.00 \odot 220 & -2.754058 \\ \mathrm{H} & -2.361937 & 0.001144 & -4.366223 \\ \mathrm{O} & -3.110098 & -0.00 \odot 869 & 1.103060 \\ \mathrm{O} & -5.002649 & -0.00 \odot 841 & -0.016561\end{array}$

$\begin{array}{rrr}\text { Frequencies }(\mathrm{cm}-1): & & \\ 27.1105 & 30.3956 & 54.4465 \\ 71.9843 & 112.9552 & 117.4157 \\ 151.7204 & 201.0579 & 206.5968 \\ 236.4976 & 290.1733 & 320.7836 \\ 369.1973 & 425.7231 & 429.8148 \\ 468.6055 & 471.3867 & 495.2689 \\ 536.4473 & 558.0176 & 561.0371 \\ 590.7770 & 593.6783 & 646.7165 \\ 659.3514 & 691.1287 & 720.0625 \\ 736.4481 & 741.2366 & 764.3712 \\ 795.7283 & 816.0433 & 823.2215 \\ 859.2237 & 885.4697 & 887.7268 \\ 904.7584 & 919.0791 & 947.3392 \\ 985.6069 & 986.5804 & 994.3579 \\ 1045.6626 & 1053.3666 & 1080.2865 \\ 1130.4369 & 1161.7739 & 1193.3565 \\ 1203.6581 & 1214.0355 & 1270.9964 \\ 1284.2928 & 1300.8726 & 1355.2257 \\ 1379.4175 & 1393.6778 & 1406.8154 \\ 1453.9254 & 1468.9086 & 1501.9630 \\ 1519.0474 & 1557.9035 & 1603.2283 \\ 1610.9389 & 1631.7666 & 1645.8029 \\ 1675.4568 & 3113.9419 & 3187.8763 \\ 3196.1480 & 3207.2474 & 3215.4593 \\ 3238.4809 & 3277.6740 & 3307.7995\end{array}$

Cis -4

Energy: -1233.41725440 hartree 


\begin{tabular}{|c|c|c|c|}
\hline \multicolumn{4}{|c|}{ Geometry (angstrom): } \\
\hline$S$ & -0.627677 & -0.368659 & 1.334642 \\
\hline C & $-\odot .639161$ & $-\odot .093765$ & 3.069039 \\
\hline C & 0.661248 & 0.181625 & 3.565597 \\
\hline C & 1.651733 & 0.126671 & 2.538588 \\
\hline C & 1.160633 & -0.131192 & 1. 280191 \\
\hline C & -1.751076 & $-\odot .131835$ & 3.913853 \\
\hline C & -1.560250 & 0.119689 & 5.269452 \\
\hline C & -0.279428 & 0.400275 & 5.780535 \\
\hline C & 0.825347 & ๑. 431254 & 4.942803 \\
\hline $\mathrm{H}$ & 1.815154 & 0.643454 & 5.337888 \\
\hline $\mathrm{H}$ & -0.157255 & 0.592315 & 6.842593 \\
\hline $\mathrm{H}$ & -2.413983 & $\odot .099324$ & 5.940893 \\
\hline $\mathrm{H}$ & -2.741062 & -0.348869 & 3.523731 \\
\hline $\mathrm{H}$ & 2.714644 & ○. 259123 & 2.705163 \\
\hline $\mathrm{N}$ & 1.990455 & -0.280588 & 0.209681 \\
\hline C & 1.770915 & -0.286542 & -1.058160 \\
\hline $\mathrm{H}$ & 2.631477 & -0.548878 & -1.677859 \\
\hline C & 0.606760 & 0.037491 & -1.867910 \\
\hline C & -0.568948 & 0.756363 & -1.736644 \\
\hline C & -1.180876 & 0.790129 & -3.012575 \\
\hline C & -0.334867 & ๑. 096239 & -3.835394 \\
\hline 0 & 0.747630 & -0.357450 & -3.178252 \\
\hline $\mathrm{H}$ & -0.936319 & 1.215767 & -0.832557 \\
\hline $\mathrm{H}$ & -2.109032 & 1.257913 & -3.302882 \\
\hline $\mathrm{N}$ & -0.435887 & -0.196683 & -5.231610 \\
\hline 0 & ○. 469762 & -0.843966 & -5.753556 \\
\hline 0 & -1.444260 & ๑. 237777 & -5.797443 \\
\hline
\end{tabular}

$\begin{array}{rrr}\text { Frequencies }(\mathrm{cm}-1): & \\ 19.2868 & 22.7684 & 60.1593 \\ 91.4622 & 100.3388 & 125.7232 \\ 161.5113 & 179.4181 & 196.9761 \\ 249.6469 & 277.2665 & 307.5484 \\ 364.9106 & 418.7841 & 432.5854 \\ 453.6678 & 481.3271 & 493.1433 \\ 534.5418 & 556.0458 & 576.0658 \\ 592.4939 & 598.1045 & 647.1977 \\ 660.3790 & 683.0115 & 717.7792 \\ 734.5293 & 746.1335 & 763.5028 \\ 790.7841 & 821.0843 & 832.2827 \\ 855.9534 & 879.7087 & 888.3289 \\ 913.2617 & 927.7499 & 945.2478 \\ 982.8107 & 985.2241 & 998.1370 \\ 1047.6302 & 1059.2461 & 1077.9643 \\ 1130.0103 & 1161.2152 & 1191.7925 \\ 1194.1959 & 1213.1898 & 1225.3784 \\ 1286.0162 & 1299.8674 & 1349.3027 \\ 1376.9754 & 1394.7077 & 1412.9605 \\ 1463.2589 & 1486.3574 & 1503.4598 \\ 1506.6225 & 1563.2023 & 1609.3947 \\ 1616.3127 & 1639.6150 & 1646.2035 \\ 1684.3214 & 3133.8116 & 3189.8162 \\ 3197.5472 & 3207.4892 & 3216.4102 \\ 3239.4482 & 3300.2394 & 3310.7480\end{array}$




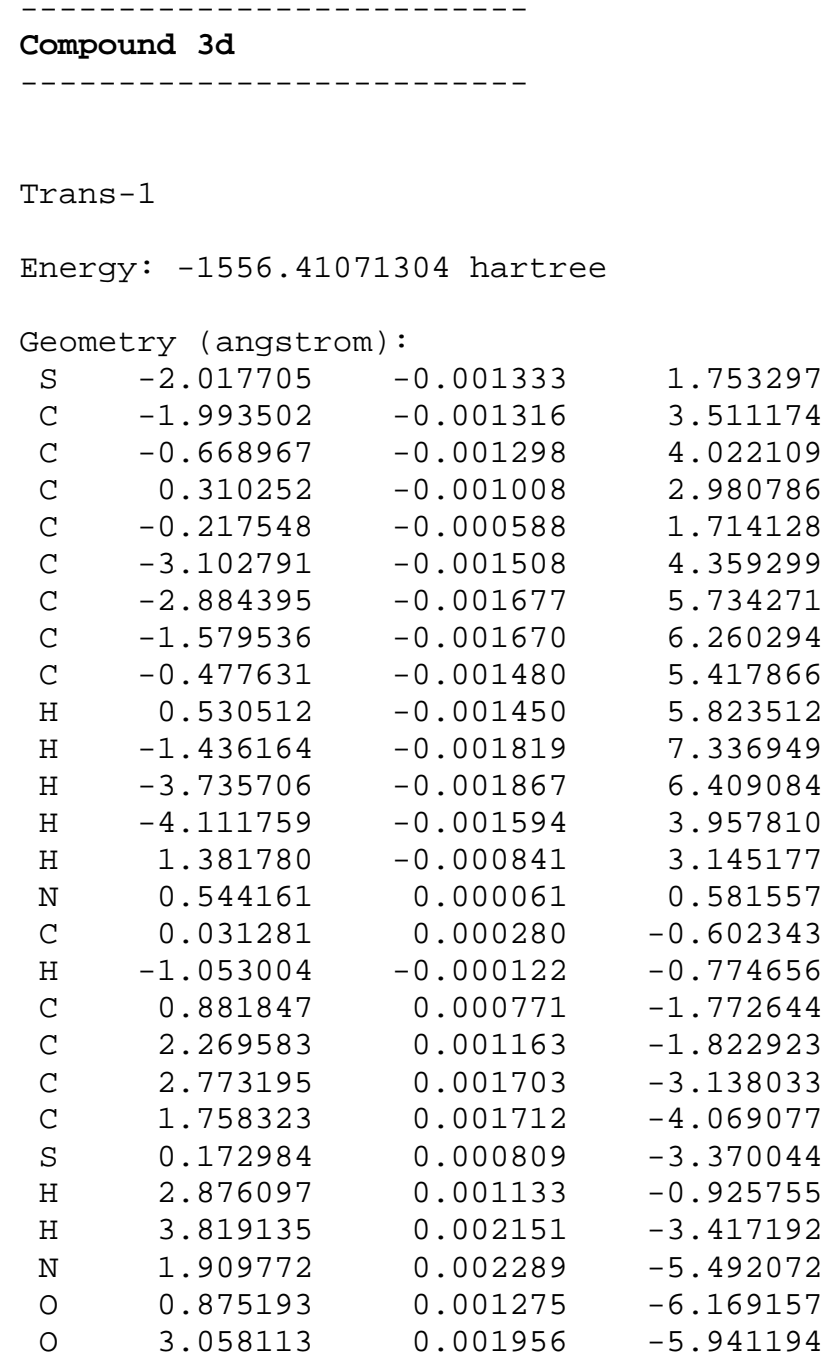

$\begin{array}{rrr}\text { Frequencies }(\mathrm{cm}-1): & & \\ 26.0461 & 34.1384 & 42.3041 \\ 74.2457 & 93.9207 & 136.0339 \\ 168.3358 & 173.1719 & 187.0839 \\ 210.8165 & 237.1128 & 298.4815 \\ 346.6959 & 361.0756 & 397.2396 \\ 444.3639 & 447.6789 & 475.8543 \\ 491.0726 & 500.3894 & 536.4039 \\ 540.2885 & 565.4950 & 600.3567 \\ 638.3203 & 660.5743 & 667.9958 \\ 717.1576 & 733.3603 & 741.3915 \\ 755.7278 & 763.5043 & 819.7213 \\ 841.1906 & 852.1064 & 854.4972 \\ 880.7750 & 885.8804 & 938.9495 \\ 945.0359 & 955.6926 & 985.0943 \\ 1046.1106 & 1050.6260 & 1077.8254 \\ 1136.3339 & 1160.0420 & 1162.2020 \\ 1191.2577 & 1203.5611 & 1228.8300 \\ 1255.8959 & 1286.3843 & 1345.4788 \\ 1372.5601 & 1380.0187 & 1397.3167\end{array}$




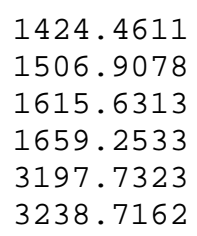

Trans - 2

Energy: -1556.41331406 hartree

Geometry (angstrom) :

\begin{tabular}{|c|c|c|c|}
\hline S & -2.538286 & -0.000417 & 1.380231 \\
\hline C & -2.520605 & -0.001282 & 3.138533 \\
\hline C & -1.197570 & -0.000994 & 3.653434 \\
\hline C & -0.214483 & $-\odot . \odot \odot \odot 337$ & 2.615460 \\
\hline C & -0.738714 & -0.000305 & 1.347778 \\
\hline C & -3.632485 & $-\odot .001987$ & 3.983150 \\
\hline C & -3.418261 & -0.002417 & 5.358861 \\
\hline C & -2.115045 & -0.002136 & 5.888737 \\
\hline C & -1.010515 & -0.001434 & 5.049656 \\
\hline $\mathrm{H}$ & -0.003618 & -0.001237 & 5.458267 \\
\hline $\mathrm{H}$ & -1.974860 & -0.002469 & 6.965813 \\
\hline $\mathrm{H}$ & -4.271592 & -0.002934 & 6.031132 \\
\hline $\mathrm{H}$ & -4.640310 & -0.002136 & 3.578697 \\
\hline $\mathrm{H}$ & 0.856601 & -0.000121 & 2.782654 \\
\hline $\mathrm{N}$ & ๑. 024977 & ๑. 000002 & ๑. 216724 \\
\hline C & -0.477885 & ๑. . 000186 & -0.971002 \\
\hline $\mathrm{H}$ & -1.559126 & 0. .000152 & -1.162686 \\
\hline C & 0.364699 & ๑. . 000605 & -2.140754 \\
\hline C & -0.053162 & ๑. . $00 \odot 9 \odot 2$ & -3.464300 \\
\hline C & 1.017689 & ๑ . 001281 & -4.383221 \\
\hline C & 2.228468 & ๑ . 001294 & -3.731618 \\
\hline S & 2.106153 & 0.000773 & -1.999643 \\
\hline $\mathrm{H}$ & -1.099382 & ๑. .000845 & -3.750637 \\
\hline $\mathrm{H}$ & 0.930370 & ๑. . 001562 & -5.461951 \\
\hline $\mathrm{N}$ & 3.519307 & ๑. .001666 & -4.356993 \\
\hline 0 & 4.505423 & $\odot .0 \odot 1592$ & -3.612676 \\
\hline 0 & 3.553736 & ๑ . 001995 & -5.589462 \\
\hline
\end{tabular}

Frequencies (cm-1):

$$
\begin{array}{r}
28.5988 \\
72.2182 \\
164.4306 \\
211.0567 \\
350.5623 \\
443.3383 \\
490.9449 \\
547.4556 \\
642.8021 \\
715.6983 \\
741.3825 \\
823.2337 \\
881.4642 \\
944.6900 \\
1048.4380
\end{array}
$$

$$
\begin{aligned}
& 1462.9478 \\
& 1561.8756 \\
& 1617.7717 \\
& 3061.8881 \\
& 3208.1511 \\
& 3252.7830
\end{aligned}
$$

1489.7193

1586.4833

1642.0388

3189.8747

3216.7590

3267.5510
38.6278

116.0765

191.6827

323.7386

397.9371

482.8067

538.1181

603.9756

701.5865

733.4217

787.7017

855.3710

924.9487

985.3743

1077.1512 


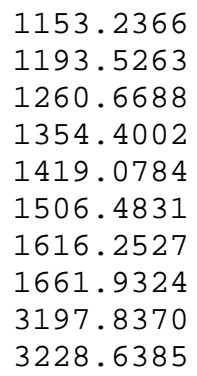

1159.8329

1219.4222

1286.0366

1375.7110

1465.9795

1565.6704

1620.0986

3062.9048

3207.8573

3240.1387
1170.9716

1246.7732

1339.9570

1383.2626

1493.9249

1591.7112

1643.6008

3190.0365

3216.6050

3268.3606

Trans - 3

Energy: -1556.40616575 hartree

Geometry (angstrom):

$\begin{array}{rrrr}\text { S } & -3.055811 & -0.436361 & 1.074218 \\ \mathrm{C} & -3.083838 & -0.414286 & 2.827348 \\ \mathrm{C} & -1.770282 & -0.343662 & 3.365825 \\ \mathrm{C} & -0.768840 & -0.285433 & 2.345188 \\ \mathrm{C} & -1.287094 & -0.306517 & 1.073037 \\ \mathrm{C} & -4.210041 & -0.459976 & 3.654292 \\ \mathrm{C} & -4.021657 & -0.446475 & 5.032107 \\ \mathrm{C} & -2.727958 & -0.384972 & 5.584302 \\ \mathrm{C} & -1.609808 & -0.335338 & 4.766184 \\ \mathrm{H} & -0.612281 & -0.284534 & 5.194650 \\ \mathrm{H} & -2.607069 & -0.375536 & 6.663798 \\ \mathrm{H} & -4.885292 & -0.486420 & 5.689825 \\ \mathrm{H} & -5.209155 & -0.510049 & 3.231433 \\ \mathrm{H} & 0.295713 & -0.270771 & 2.553631 \\ \mathrm{~N} & -0.675484 & -0.312430 & -0.159789 \\ \mathrm{C} & 0.493331 & 0.207585 & -0.315946 \\ \mathrm{H} & 1.016122 & 0.730054 & 0.495625 \\ \mathrm{C} & 1.168491 & 0.131194 & -1.595331 \\ \mathrm{C} & 0.723962 & -0.473754 & -2.762831 \\ \mathrm{C} & 1.636595 & -0.350043 & -3.828931 \\ \mathrm{C} & 2.763380 & 0.345806 & -3.451955 \\ \mathrm{~S} & 2.739687 & 0.867261 & -1.800304 \\ \mathrm{H} & -0.231273 & -0.981066 & -2.818287 \\ \mathrm{H} & 1.501037 & -0.742359 & -4.828693 \\ \mathrm{~N} & 3.892849 & 0.650509 & -4.279315 \\ \mathrm{O} & 4.816245 & 1.286523 & -3.759154 \\ \mathrm{O} & 3.862652 & 0.257847 & -5.447172\end{array}$

Frequencies (cm-1):

$\begin{array}{rr}25.1414 & 31.1670 \\ 80.2160 & 92.6820 \\ 170.1649 & 177.6702 \\ 204.1100 & 242.1863 \\ 331.2769 & 361.8960 \\ 436.4720 & 448.9340 \\ 493.0667 & 500.3458 \\ 540.1936 & 568.0039 \\ 639.8427 & 651.6275 \\ 729.9222 & 733.4718 \\ 756.3021 & 759.9945\end{array}$

39.5157

127.2762

192.2909

289.1811

396.7561

479.3643

526.0381

602.7892

713.0204

741.4500

820.1817 


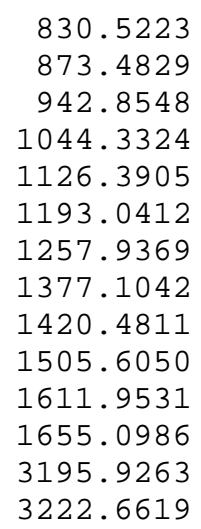

843.2421

902.2655

981.3520

1049.7874

1158.5790

1202.5236

1286.4938

1381.3863

1460.4575

1551.8423

1619.5730

3065.6189

3205.8408

3253.3985
864.3055

941.5734

983.4757

1078.8820

1162.8236

1225.6014

1352.5921

1398.3388

1487.3394

1575.3113

1640. 9399

3187.5581

3215.3779

3268.5843

Trans -4

Energy: -1556.40844985 hartree

Geometry (angstrom):

\begin{tabular}{|c|c|c|c|}
\hline S & -2.563007 & -0.117185 & 1.326114 \\
\hline C & -2.591306 & -0.080032 & 3.078517 \\
\hline C & -1.278425 & $\odot .0 \odot 2409$ & 3.617240 \\
\hline C & -0.277220 & 0.056200 & 2.596309 \\
\hline C & -0.795627 & 0.020488 & 1.32456 \\
\hline C & -3.717643 & -0.125751 & 3.905508 \\
\hline C & -3.530158 & -0.099793 & 5.28314 \\
\hline C & -2.237095 & -0.026033 & 5.83559 \\
\hline C & -1.118888 & $\odot .023536$ & 5.01764 \\
\hline $\mathrm{H}$ & -0.121864 & ๑. .083056 & 5.4462 \\
\hline $\mathrm{H}$ & -2.116870 & -0.007371 & 6.91504 \\
\hline $\mathrm{H}$ & -4.393949 & -0.139349 & 5.94069 \\
\hline $\mathrm{H}$ & -4.716238 & -0.185194 & 3.4825 \\
\hline $\mathrm{H}$ & ๑. 787294 & ○. . 079824 & 2.80442 \\
\hline $\mathrm{N}$ & -0.183910 & $\odot . \odot \odot 8938$ & ๑. . 09194 \\
\hline C & ๑. 988802 & 0.516114 & -0.07393 \\
\hline $\mathrm{H}$ & 1.532276 & 1.034960 & 0.726660 \\
\hline C & 1.663346 & 0.443306 & -1.34786 \\
\hline C & 2.912989 & 0.962400 & -1.65458 \\
\hline C & 3. 293981 & ๑. 733104 & -2.99414 \\
\hline C & 2.318898 & 0.040754 & -3.67220 \\
\hline S & $\odot .924190$ & -0.350889 & -2.71635 \\
\hline $\mathrm{H}$ & 3.524960 & 1.490238 & -0.93104 \\
\hline $\mathrm{H}$ & 4.220852 & 1.048372 & -3.45535 \\
\hline $\mathrm{N}$ & 2.377025 & $-\odot .358407$ & -5.05021 \\
\hline 0 & 1.405012 & -0.973971 & -5.49883 \\
\hline 0 & 3.390349 & -0.059689 & -5.68521 \\
\hline
\end{tabular}

Frequencies (cm-1):

$\begin{array}{rr}21.4961 & 34.6734 \\ 76.2274 & 97.6527 \\ 170.1535 & 188.9601 \\ 202.3001 & 252.7845 \\ 337.0826 & 364.3709 \\ 437.7080 & 449.4371 \\ 488.8543 & 496.9337\end{array}$

40.3334

112.2580

196.1771

310.7743

399.3450

487.3630

540.7657 


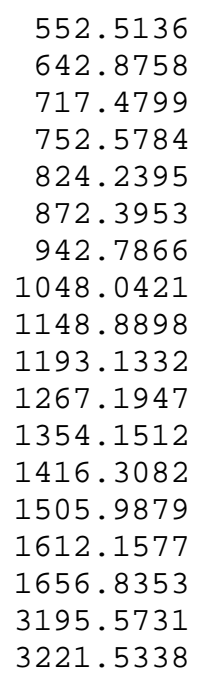

$$
\begin{array}{r}
569.0452 \\
671.2620 \\
733.3360 \\
759.8998 \\
825.4893 \\
891.8674 \\
979.0739 \\
1062.1585 \\
1158.2149 \\
1217.6312 \\
1287.3203 \\
1380.1817 \\
1461.8684 \\
1551.9608 \\
1622.3816 \\
3065.5754 \\
3205.7449 \\
3229.3078
\end{array}
$$

606.1147

693.0543

741.2802

808.5991

838.1141

926.7012

983.5521

1079.1477

1165.2839

1244.2698

1348.0975

1384.8150

1494.6817

1584.3169

1643.0557

3187.3587

3215.2359

3269.5919

Cis -1

Energy: -1556.39573139 hartree

Geometry (angstrom):

$\begin{array}{lrrr}\text { S } & -2.816352 & -1.815723 & -0.173398 \\ \mathrm{C} & -2.799589 & -1.768920 & 1.583288 \\ \mathrm{C} & -1.475823 & -1.656938 & 2.083519 \\ \mathrm{C} & -0.503413 & -1.565695 & 1.032479 \\ \mathrm{C} & -1.052709 & -1.610427 & -0.223060 \\ \mathrm{C} & -3.903179 & -1.823741 & 2.437933 \\ \mathrm{C} & -3.679919 & -1.790112 & 3.811350 \\ \mathrm{C} & -2.374913 & -1.693037 & 4.326835 \\ \mathrm{C} & -1.279123 & -1.626333 & 3.477679 \\ \mathrm{H} & -0.272452 & -1.546260 & 3.879681 \\ \mathrm{H} & -2.224795 & -1.668298 & 5.402481 \\ \mathrm{H} & -4.525103 & -1.843406 & 4.491677 \\ \mathrm{H} & -4.911395 & -1.902015 & 2.041654 \\ \mathrm{H} & 0.565720 & -1.505106 & 1.204562 \\ \mathrm{~N} & -0.455189 & -1.728814 & -1.458984 \\ \mathrm{C} & 0.477668 & -0.988712 & -1.940424 \\ \mathrm{H} & 0.872794 & -1.328161 & -2.901956 \\ \mathrm{C} & 1.067837 & 0.264319 & -1.467518 \\ \mathrm{C} & 0.729091 & 1.124523 & -0.430866 \\ \mathrm{C} & 1.510669 & 2.298333 & -0.402443 \\ \mathrm{C} & 2.439613 & 2.312465 & -1.416383 \\ \mathrm{~S} & 2.380929 & 0.916246 & -2.431252 \\ \mathrm{H} & -0.057148 & 0.909150 & 0.279944 \\ \mathrm{H} & 1.417204 & 3.101294 & 0.317528 \\ \mathrm{~N} & 3.404125 & 3.345766 & -1.670567 \\ \mathrm{O} & 4.162099 & 3.182217 & -2.631641 \\ \mathrm{O} & 3.403167 & 4.317961 & -0.914440\end{array}$




$\begin{array}{rrr}\text { Frequencies }(\mathrm{cm}-1): & \\ 20.4361 & 26.9687 & 44.8977 \\ 84.3668 & 94.2510 & 113.6323 \\ 138.0222 & 186.7223 & 199.6215 \\ 240.5267 & 256.1089 & 288.1334 \\ 354.9440 & 380.3531 & 426.6532 \\ 445.1749 & 457.3179 & 482.8325 \\ 493.3883 & 500.1373 & 535.1552 \\ 557.6919 & 570.0904 & 617.7826 \\ 632.6419 & 647.7248 & 709.1412 \\ 728.9186 & 733.0860 & 741.5231 \\ 753.0517 & 759.7072 & 821.3883 \\ 824.8556 & 848.2266 & 856.7200 \\ 872.2810 & 884.4029 & 939.7593 \\ 941.6488 & 955.3703 & 981.4235 \\ 1040.9210 & 1050.2460 & 1076.7189 \\ 1103.4692 & 1147.7641 & 1162.3546 \\ 1167.9619 & 1192.1375 & 1220.0398 \\ 1261.6983 & 1286.1342 & 1345.8937 \\ 1374.6535 & 1378.9379 & 1393.8361 \\ 1462.8004 & 1472.2598 & 1490.4481 \\ 1505.3004 & 1558.5373 & 1575.9300 \\ 1614.0173 & 1624.8429 & 1647.6122 \\ 1677.6467 & 3117.0613 & 3186.4669 \\ 3194.8322 & 3204.7944 & 3214.4791 \\ 3225.9203 & 3261.8976 & 3273.0601\end{array}$

\section{Cis-2}

Energy: -1556.39743423

Geometry (angstrom) :

$\begin{array}{lrrr}\text { S } & -2.544493 & -1.457162 & -0.914896 \\ \text { C } & -2.598930 & -1.603257 & 0.836789 \\ \text { C } & -1.301418 & -1.786434 & 1.380177 \\ \text { C } & -0.281299 & -1.767519 & 0.369680 \\ \text { C } & -0.773942 & -1.576363 & -0.894769 \\ \text { C } & -3.732807 & -1.547675 & 1.650183 \\ \text { C } & -3.569716 & -1.699292 & 3.024234 \\ \text { C } & -2.293287 & -1.894123 & 3.580850 \\ \text { C } & -1.165411 & -1.938102 & 2.772892 \\ \text { H } & -0.179332 & -2.080724 & 3.206886 \\ \text { H } & -2.189619 & -2.007927 & 4.656197 \\ \text { H } & -4.441007 & -1.668707 & 3.672262 \\ \text { H } & -4.719704 & -1.399119 & 1.221711 \\ \text { H } & 0.770323 & -1.937006 & 0.573090 \\ \text { N } & -0.137146 & -1.658791 & -2.117227 \\ \text { C } & 0.866923 & -0.944001 & -2.474388 \\ \text { H } & 1.288905 & -1.194493 & -3.451948 \\ \mathrm{C} & 1.532864 & 0.210776 & -1.876138 \\ \mathrm{C} & 2.514455 & 0.913254 & -2.564025 \\ \mathrm{C} & 2.963919 & 2.070460 & -1.894421 \\ \mathrm{C} & 2.308529 & 2.225894 & -0.698364 \\ \mathrm{~S} & 1.151267 & 0.979249 & -0.350798 \\ \mathrm{H} & 2.878154 & 0.598691 & -3.536543 \\ \mathrm{H} & 3.717523 & 2.760548 & -2.250738 \\ \mathrm{~N} & 2.521325 & 3.286294 & 0.248924 \\ \mathrm{O} & 1.836199 & 3.267046 & 1.275684 \\ \mathrm{O} & 3.371032 & 4.133093 & -\Theta .030420\end{array}$




$\begin{array}{rrr}\text { Frequencies }(\mathrm{cm}-1): & \\ 22.1825 & 32.6361 & 41.2637 \\ 76.7654 & 97.7742 & 104.0250 \\ 142.6352 & 188.0892 & 202.4753 \\ 240.7295 & 266.2878 & 303.2698 \\ 354.8774 & 381.0578 & 428.3714 \\ 447.1827 & 456.2689 & 482.3851 \\ 493.6107 & 509.5330 & 547.3912 \\ 566.1908 & 579.7397 & 608.8216 \\ 633.2808 & 665.6630 & 692.2775 \\ 714.6834 & 733.1339 & 741.0383 \\ 748.7083 & 761.1745 & 796.4320 \\ 824.7607 & 829.8304 & 838.5838 \\ 871.5698 & 882.5694 & 928.0272 \\ 939.6450 & 960.2480 & 981.4908 \\ 1048.5672 & 1064.4339 & 1076.4693 \\ 1123.8498 & 1148.7414 & 1162.5848 \\ 1192.3825 & 1219.9929 & 1228.0201 \\ 1245.3283 & 1286.7149 & 1345.0155 \\ 1358.3156 & 1377.3535 & 1387.3832 \\ 1454.8100 & 1470.3776 & 1490.4595 \\ 1504.5911 & 1559.7815 & 1578.0714 \\ 1615.4523 & 1625.7446 & 1648.2661 \\ 1681.9651 & 3118.0965 & 3187.7686 \\ 3195.8673 & 3205.6807 & 3215.1964 \\ 3227.6518 & 3228.1209 & 3270.4669\end{array}$

Cis -4

Energy: -1556.39589036 hartree

Geometry (angstrom):

\begin{tabular}{|c|c|c|c|}
\hline 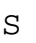 & $-\odot .581944$ & -0.472717 & 1.320620 \\
\hline$C$ & $-\odot .919517$ & $-\odot .329662$ & 3.039050 \\
\hline C & 0.250252 & -0.026005 & 3.782453 \\
\hline C & 1.410314 & 0.042129 & 2.951750 \\
\hline C & 1.167799 & $-\odot .149163$ & 1.612223 \\
\hline c & -2.160846 & -0.480382 & 3. 661792 \\
\hline C & -2.232965 & -0.316052 & 5.042238 \\
\hline C & -1.084818 & -0.010546 & 5.796143 \\
\hline C & 0.148849 & 0.133196 & 5.178648 \\
\hline H & 1.036672 & ๑.365192 & 5.760811 \\
\hline $\mathrm{H}$ & -1.167673 & 0.111908 & 6.872221 \\
\hline $\mathrm{H}$ & -3.190952 & -0.424904 & 5.542827 \\
\hline $\mathrm{H}$ & -3.049618 & $-\odot .716629$ & 3.084107 \\
\hline $\mathrm{H}$ & 2.416488 & 0.212014 & 3.317817 \\
\hline $\mathrm{N}$ & 2.187875 & -0.194634 & 0.704519 \\
\hline C & 2.181244 & -0.150342 & -0.581320 \\
\hline $\mathrm{H}$ & 3.151362 & $-\odot .352089$ & -1.043785 \\
\hline C & 1.121164 & 0.168883 & -1.541061 \\
\hline c & 0.063145 & 1.061265 & -1.449587 \\
\hline C & $-\odot .669740$ & 1.186894 & -2.650023 \\
\hline C & -0.150091 & $\odot .392272$ & -3.642974 \\
\hline S & 1.237724 & -0.517734 & -3.150149 \\
\hline H & -0.156780 & 1.625548 & $-\odot .552926$ \\
\hline $\mathrm{H}$ & -1.529505 & 1.826990 & -2.800786 \\
\hline $\mathrm{N}$ & $-\odot .650588$ & 0.269999 & -4.982304 \\
\hline 0 & $-\odot .048975$ & -0.498742 & -5.739226 \\
\hline 0 & -1.640052 & 0.940829 & -5.280690 \\
\hline
\end{tabular}

S65 


$\begin{array}{rrr}\text { Frequencies }(\mathrm{cm}-1): & \\ 18.2345 & 20.8696 & 55.4646 \\ 87.9679 & 104.2196 & 111.5313 \\ 152.9397 & 167.2323 & 196.4827 \\ 236.2189 & 253.7894 & 298.8626 \\ 350.3123 & 389.1586 & 415.1198 \\ 442.0814 & 467.0509 & 475.9488 \\ 492.6275 & 496.4799 & 546.7560 \\ 555.7407 & 566.0178 & 615.1542 \\ 635.6458 & 641.1208 & 672.9030 \\ 717.8784 & 734.3214 & 741.2180 \\ 752.0491 & 763.5522 & 819.2327 \\ 827.6612 & 846.0879 & 856.8749 \\ 879.5893 & 888.1097 & 911.4410 \\ 935.8908 & 944.6482 & 984.5873 \\ 1041.5342 & 1049.6379 & 1077.1448 \\ 1102.4703 & 1150.7439 & 1161.0131 \\ 1166.9972 & 1192.1215 & 1213.7779 \\ 1260.7928 & 1285.9708 & 1348.2613 \\ 1371.5902 & 1379.8581 & 1391.7014 \\ 1462.1497 & 1475.0830 & 1484.2292 \\ 1505.3233 & 1561.4517 & 1577.5537 \\ 1616.0514 & 1622.7856 & 1644.8100 \\ 1671.1124 & 3114.3457 & 3189.3167 \\ 3197.0464 & 3207.2061 & 3216.0999 \\ 3238.4168 & 3257.8027 & 3270.5074\end{array}$

